\title{
The asymptotics of monotone subsequences of involutions
}

\author{
Jinho Baik* and Eric M. Rains ${ }^{\dagger \ddagger}$
}

January 31, 2001

\begin{abstract}
We compute the limiting distributions of the lengths of the longest monotone subsequences of random (signed) involutions with or without conditions on the number of fixed points (and negated points) as the sizes of the involutions tend to infinity. The resulting distributions are, depending on the number of fixed points, (1) the Tracy-Widom distributions for the largest eigenvalues of random GOE, GUE, GSE matrices, (2) the normal distribution, or (3) new classes of distributions which interpolate between pairs of the TracyWidom distributions. We also consider the second rows of the corresponding Young diagrams. In each case the convergence of moments is also shown. The proof is based on the algebraic work of the authors in [7] which establishes a connection between the statistics of random involutions and a family of orthogonal polynomials, and an asymptotic analysis of the orthogonal polynomials which is obtained by extending the Riemann-Hilbert analysis for the orthogonal polynomials by Deift, Johansson and the first author in [3].
\end{abstract}

\section{Introduction}

\section{$\beta$-Plancherel measure}

In the last few years, it has been observed by many authors that there are certain connections between random permutations and/or Young tableaux, and random matrices. One of the earliest clues to this relationship appeared in the work of Regev [39] in 1981. A Young diagram, or equivalently a partition $\lambda=\left(\lambda_{1}, \lambda_{2}, \cdots\right) \vdash n$ $\left(\lambda_{1} \geq \lambda_{2} \geq \cdots, \sum \lambda_{j}=n\right)$ is an array of $n$ boxes with top and left adjusted as in the first picture of Figure 1, which represents the example $\lambda=(4,3,1) \vdash 8$. A standard Young tableau $Q$ is a filling of the diagram $\lambda$ by numbers $1,2, \cdots, n$ such that numbers are increasing along each row and along each column. In this case, we say that the tableau $Q$ has the shape $\lambda$. The second picture in Figure 1 is an example of a standard Young tableau with shape $\lambda=(4,3,1)$. Let $d_{\lambda}$ denote the number of standard Young tableaux of shape $\lambda$. A result of

*Princeton University and Institite for Advanced Study, New Jersey, jbaik@math.princeton.edu

${ }^{\dagger}$ AT\&T Research, New Jersey, rains@research.att.com

¥1991 Mathematics Subject Classification. Primary 60C05 ; Secondary 45E05, 05A05 

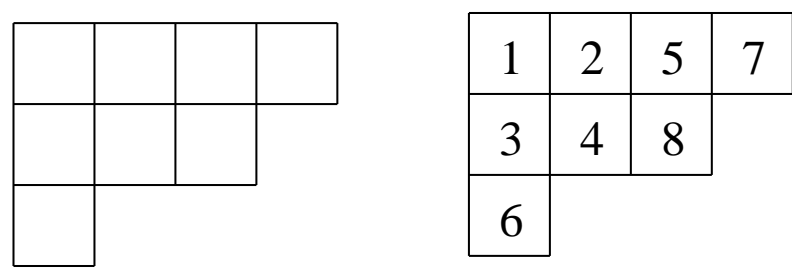

Figure 1: Young diagram and standard Young tableau

[39] is that for fixed $\beta>0$ and fixed $l$, as $n \rightarrow \infty$,

$$
\sum_{\substack{\lambda \vdash n \\ \lambda_{1} \leq l}}\left(d_{\lambda}\right)^{\beta} \sim\left[\frac{l^{l^{2} / 2} l^{n}}{(\sqrt{2 \pi})^{(l-1) / 2} n^{(l-1)(l+2) / 4}}\right]^{\beta} \frac{n^{(l-1) / 2}}{l !} \int_{\mathbb{R}^{l}} e^{-\frac{1}{2} \beta l \sum_{j} x_{j}^{2}} \prod_{j<k}\left|x_{j}-x_{k}\right|^{\beta} d^{l} x .
$$

The multiple integral on the right hand side is called the Selberg integral, which can be computed exactly for each $\beta$ in terms of the Gamma function. In particular, when $\beta=1,2,4$, this integral is the normalization constant of the eigenvalue density of a random matrix taken from the Gaussian orthogonal ensemble (GOE), Gaussian unitary ensemble (GUE), Gaussian symplectic ensemble (GSE), respectively (see e.g. 36]). Motivated by this result, we define the $\beta$-Plancherel measure on the set $Y_{n}$ of Young diagrams (or partitions) of size $n$ by

$$
M_{n}^{\beta}(\lambda):=\frac{d_{\lambda}^{\beta}}{\sum_{\mu \vdash n} d_{\mu}^{\beta}}, \quad \lambda \in Y_{n} .
$$

A natural question is the limiting statistics of a random $\lambda \in Y_{n}$ under $M_{n}^{\beta}$ as $n \rightarrow \infty$.

The case when $\beta=2$ is quite well studied. In this case, $M_{n}^{2}$ is called the Plancherel measure which arises in the representation theory of the symmetric group $S_{n}$. Denote by $L_{n}^{(k)}$ the random variable $\lambda_{k}$ under the Plancherel measure $M_{n}^{2}$, and set $L_{n}=L_{n}^{(1)}$. In 1977, the limiting expected shape of $\lambda$ under $M_{n}^{2}$ is obtained in [48], also independently in [35] for the so-called Poissonized Plancherel measure. In particular, it is shown that

$$
\lim _{n \rightarrow \infty} \frac{\mathrm{E}\left(L_{n}\right)}{\sqrt{n}}=2 .
$$

A central limit theorem for $L_{n}$ is then obtained by [3]:

$$
\lim _{n \rightarrow \infty} \operatorname{Pr}\left(\frac{L_{n}-2 \sqrt{n}}{n^{1 / 6}}\right)=F_{2}(x),
$$

where $F_{2}$ is the so-called Tracy-Widom distribution function, which is expressed in terms of a solution to the Painlevé II equation (see Definition 2 in Section 2 for the definition). The connection to random matrix theory comes from this function $F_{2}$ : in 1994, Tracy and Widom 445 proved that under proper centering and scaling (which is different from the scaling for $L_{n}$ in (1.4)), the largest eigenvalue of a random matrix taken from the Gaussian unitary ensemble (GUE) has the same limiting distribution given by $F_{2}$. In other words, after proper centering and scaling, the first row of a random Young diagram under the Plancherel measure behaves statistically for large $n$ like the largest eigenvalue of a random GUE matrix. Then in the same paper [3], it was conjectured that $L_{n}^{(k)}$ of a random $\lambda \in Y_{n}$ under $M_{n}^{2}$ have the same limiting distribution as the $k^{t h}$ largest 
eigenvalue of a random GUE matrix for each $k$. This conjecture was supported by numerical simulations of Odlyzko and the second author, and was proved to be true for the second row $L_{n}^{(2)}$ in 跑. The full conjecture for the general row $L_{n}^{(k)}$ was subsequently proved by [38], [10] and [32], independently. The authors in [38, 10, 32] proved the convergence in joint distribution for general rows, and also in [10, 32], the authors obtained discrete sine kernel representations for the so-called bulk scaling limit of correlation functions, an analogue of the sine kernel that appears in the GUE matrix case. In [3] and [4], in addition to convergence in distribution, the authors also proved convergence of moments for $L_{n}^{(1)}$ and $L_{n}^{(2)}$, respectively. Convergence of (joint) moments for the general rows is obtained recently by [5]. We also mention that there are many works on similar relationships between tableaux/combinatorics and GUE random matrices. See for example, 47, 9, 31, 44, 28, 34, 43]. We refer the readers to [1, 37, 12 for a survey and history of $L_{n}$, and to 36 for general reference on random matrix theory (see also the recent book [1]).

One of the main topics in this paper is the limiting statistics of $\lambda \in Y_{n}$ under $M_{n}^{1}$. From (1.1) and the results for the case when $\beta=2$, one might guess that for $\beta=1$ the limiting statistics of a random $\lambda$ is same as the limiting statistics of the eigenvalues of GOE (Gaussian orthogonal ensemble) matrices. We establish this fact for the first two rows. More precisely, we prove (see Theorems 3.4 and 3.6 together with the remark that follows), denoting by $\tilde{L}_{n}^{(k)}$ the random variable $\lambda_{k}$ of a random $\lambda \in Y_{n}$ under $M_{n}^{1}$,

$$
\lim _{n \rightarrow \infty} \operatorname{Pr}\left(\frac{\tilde{L}_{n}^{(k)}-2 \sqrt{n}}{n^{1 / 6}} \leq x\right)=F_{1}^{(k)}(x), \quad k=1,2,
$$

where $F_{1}^{(k)}(x)$ is the limiting distribution function [46] for the (scaled) $k^{\text {th }}$ largest eigenvalue of a random matrix taken from GOE. We also prove convergence of moments. As in the case of $\beta=2$, we expect that the above result should extend to the general rows $k \geq 3$, and also to the joint distributions. For general values of $\beta>0$, again from (1.1), we expect that in the large $n$ limit, the rows of a random Young diagram under $M_{n}^{\beta}$ correspond to the Coulomb charges on the real line with the quadratic potential at the inverse temperature $\beta$, which specializes to GOE, GUE, GSE eigenvalue distributions for the cases $\beta=1,2,4$, respectively. This conjecture seems natural from the perspective of the discrete Coulomb gas interpretation for the Plancherel measure case $M_{n}^{2}$ given by Johansson [31, 32].

\section{Random involutions}

The Plancherel measure $M_{n}^{2}$ has a nice combinatorial interpretation. The well-known Robinson-Schensted correspondence [40 establishes a bijection between the permutations $\pi$ of size $n$ and the pairs of standard Young tableaux $(P, Q)$ where the shape of $P$ and the shape of $Q$ are the same and the shape of $P$ (or $Q$ ), denoted by $\lambda(\pi)$, is a partition of $n$. Thus the Plancherel measure $M_{n}^{2}$ on $Y_{n}$ is the push forward of the uniform probability measure on $S_{n}$. Moreover, under this correspondence, $\lambda_{1}(\pi)$ is equal to the length of the longest increasing subsequence of $\pi$. More generally, a theorem of Greene [26] says that $\lambda_{1}(\pi)+\cdots+\lambda_{k}(\pi)$ is equal to the length of the longest so-called $k$-increasing subsequence of $\pi$. Thus the difference of the lengths of the longest $k$-increasing subsequence and the longest $(k-1)$-increasing subsequence of $\pi \in S_{n}$ under the uniform probability measure is equal to $\lambda_{k}$ of $\lambda \in Y_{n}$ under the Plancherel measure $M_{n}^{2}$ in the sense of joint distributions. Thus for example, (1.3) and (1.4) can be restated for the results on the longest increasing subsequence of a 
random permutation. On the other hand, the sum of the lengths of the first $k$ columns of $\lambda$ is equal to the length of the longest $k$-decreasing subsequence of corresponding $\pi$. But since the transpose $\lambda^{t}$ have the same statistics as $\lambda$ under $M_{n}^{2}$, the results (1.3) and (1.4) also hold for the longest decreasing subsequence of a random permutation.

The measure $M_{n}^{1}$ also has a combinatorial interpretation. If $\pi$ is mapped to $(P, Q)$ under the RobinsonSchensted correspondence, then $\pi^{-1}$ is mapped to $(Q, P)$ (see e.g. Section 5.1.4 of [33]). Therefore, the set of involutions $\pi=\pi^{-1} \in S_{n}$ is in bijection with the set of standard Young tableaux whose shapes are partitions of $n$. Consequently, the uniform probability measure on the set of involutions

$$
\tilde{S}_{n}=\left\{\pi \in S_{n}: \pi=\pi^{-1}\right\}
$$

is pushed forward to the 1-Plancherel measure $M_{n}^{1}$ on $Y_{n}$. Thus the result (1.5) for $k=1$ implies that in the large $n$ limit, the length of the longest increasing (also decreasing) subsequence of a random involution behaves statistically like the largest eigenvalue of a random GOE matrix.

An involution $\pi \in \tilde{S}_{n}$ consists only of 1 cycles and 2 cycles. It turns out that if we put a condition on the number of 1 cycles (or fixed points) of $\pi$, the limiting distribution is different. Introduce a new ensemble

$$
S_{n, m}=\left\{\pi \in \tilde{S}_{2 n+m}:|\{x: \pi(x)=x\}|=m\right\} .
$$

For an involution $\pi$, the number of fixed points is equal to the number of odd parts of $\lambda^{t}$ (see [33]). Equivalently, the number of fixed points of $\pi$ is equal to $\lambda_{1}-\lambda_{2}+\lambda_{3}-\cdots$. Thus the uniform probability measure on the set $S_{n, m}$ is push-forwarded to the measure

$$
\frac{d_{\lambda}}{\sum_{\mu \in Y_{n, m}} d_{\mu}}, \quad \lambda \in Y_{n, m}
$$

where

$$
Y_{n, m}=\left\{\lambda=\left(\lambda_{1}, \lambda_{2}, \cdots\right) \in Y_{2 n+m}: \sum_{j}(-1)^{j-1} \lambda_{j}=m\right\}
$$

Note that the rows and columns of $\lambda \in Y_{n, m}$ now have different distributions. We denote by $L_{n, m}^{\square,(k)}$ and $L_{n, m}^{\square,(k)}$ the random variables given by the lengths of the $k^{\text {th }}$ row and the $k^{\text {th }}$ column of a random $\lambda \in Y_{n, m}$ under the measure (1.8), respectively. We also set $L_{n, m}^{\square}=L_{n, m}^{\square,(1)}$ and $L_{n, m}^{\square}=L^{\square,(1)}$, the length of the longest increasing and decreasing subsequences of a random $\pi \in S_{n, m}$ under the uniform probability measure.

Set

$$
\alpha=\frac{m}{\sqrt{2 n}}
$$

The limiting distribution of $L_{n}^{\square}$ differs depending on $\alpha$. Indeed we prove in Theorem 3.1 and Theorem 9.2 below 
that

$$
\begin{aligned}
& \lim _{n \rightarrow \infty} \operatorname{Pr}\left(\frac{L_{n,[\alpha \sqrt{2 n}]}^{\square}-2 \sqrt{2 n+m}}{(2 n+m)^{1 / 6}} \leq x\right)=F_{4}(x), \quad 0 \leq \alpha<1, \\
& \lim _{n \rightarrow \infty} \operatorname{Pr}\left(\frac{L_{n,[\sqrt{2 n}]}^{\square}-2 \sqrt{2 n+m}}{(2 n+m)^{1 / 6}} \leq x\right)=F_{1}(x), \quad \alpha=1, \\
& \lim _{n \rightarrow \infty} \operatorname{Pr}\left(\frac{L_{n,[\alpha \sqrt{2 n}]}^{\square}-(\alpha+1 / \alpha) \sqrt{2 n+m}}{\sqrt{\left(1 / \alpha-1 / \alpha^{3}\right)}(2 n+m)^{1 / 4}}\right)=\operatorname{erf}(x), \quad \alpha>1,
\end{aligned}
$$

where $F_{4}$ and $F_{1}$ are the distributions for the limiting fluctuations of the largest eigenvalues of random GSE and GOE matrices respectively, and erf is the standard normal distribution. Again we also prove convergence of moments. We note that $F_{4}=F_{1}^{(2)}$; the limiting distributions of the largest eigenvalue of GSE and the second largest eigenvalue of GOE are the same (see the discussion at the end of Section 3 below.)

The role of the number of fixed points for the limiting distribution can be seen from the following point selection picture. Consider a unit square $[0,1] \times[0,1]$ in the plane and set $\delta=\{(x, x): 0 \leq x \leq 1\}$, the diagonal. Suppose we select $n$ points at random in the lower triangle $0 \leq x<y \leq 1$ and take the mirror image of the points about the diagonal $\delta$. We also select $m$ points at random on the diagonal $\delta$. Hence there is a total of $2 n+m$ points in the square. As illustrated in Figure 2, one such choice of points gives rise to a permutation $\pi$

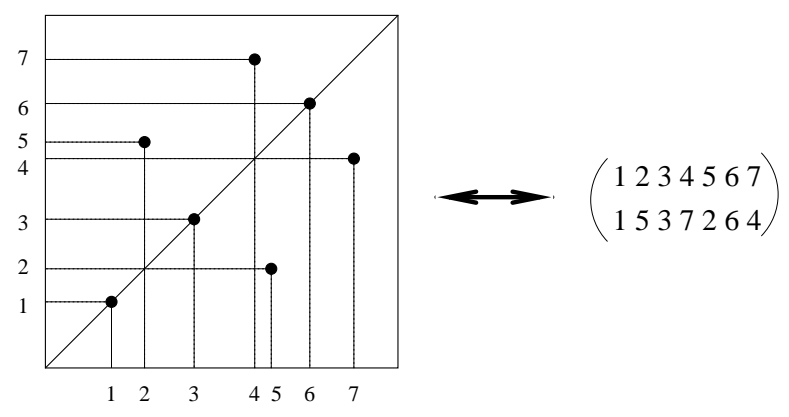

Figure 2: Point selection process

satisfying $\pi^{2}=1$ with $m$ fixed points, i.e., $\pi \in S_{n, m}$. The length $L_{n}^{\square}(\pi)$ of the longest increasing subsequence of $\pi$ is then equal to the 'length' of the longest (piecewise linear) up/right path in the square from $(0,0)$ to $(1,1)$, where the 'length' of a path is defined by the number of points on the path. The length of the longest up/right path in the above point selection process has the same distribution as $L_{n}^{\square}$. Now note that the points on $\delta$ form an increasing path. When $m$ is large comparing to $n$, there are many points on $\delta$ and we expect that the longest path consists mostly of diagonal points. Hence we are in the linear statistics situation, and thus the order of fluctuation of the length of the longest path is expected to be $(\text { mean })^{1 / 2}$ by the usual central limit theorem. On the other hand, when $m$ is small comparing to $n$, then the longest path contains few diagonal points (none if $m=0$ ) and we are in the situation of a 2-dimensional maximization problem. In this case it has been believed, and in a few cases (for example, 33, 32, 25]) it is proved, that the fluctuation has order $(\text { mean })^{1 / 3}$. (For random permutations, which have a similar interpretation as a point selection process, one can see from 
the scaling in (1.4) that the fluctuation is of order $(\text { mean })^{1 / 3}$.) Thus there must be a transition of the limiting distribution as the size of $m$ varies. The results (1.11)-(1.13) show that $\alpha=1$ is the transition point. The fixed points play the role of adding a special line in the 2-dimensional maximization problem. (See [6] for a relevant work where two special lines are added to a 2-dimensional maximization problem.) We note that when $\alpha=1$, $L_{n,[\sqrt{2 n}]}^{\square}$ in (1.12) has the same limiting distribution as $\widetilde{L}_{n}^{(1)}$ (see (1.5)). This is because the typical number of fixed points of a random involution of size $k$ is $\sqrt{k}$. Indeed the result (1.5) is proved by using (1.11)-(1.13) and taking a summation over the number of fixed points (see Section 8 below) to which only $\alpha=1$ gives the main contribution.

Once the transition point $\alpha=1$ is known, it is of interest to investigate the transition more carefully. We set

$$
\alpha=1-\frac{2 w}{(2 n)^{1 / 6}},
$$

and take $n \rightarrow \infty$ while keeping $w$ fixed. We prove that (Theorem 3.2 below) there is a one-parameter family of distribution functions $F^{\square}(x ; w)$, which is expressed in terms of the Riemann-Hilbert representation for the Painlevé II equation (see Definition 1 below), such that

$$
\lim _{n \rightarrow \infty} \operatorname{Pr}\left(\frac{L_{n,[\alpha \sqrt{2 n}]}^{\square}-2 \sqrt{2 n+m}}{(2 n+m)^{1 / 6}} \leq x\right)=F^{\square}(x ; w) .
$$

The new class of distributions $F^{\square}(x ; w)$ interpolates $F_{4}$ and $F_{1}$ as $w \rightarrow \infty$ and $w=0$ respectively, and satisfies $\lim _{w \rightarrow-\infty} F^{\square}(x ; w)=0$, so 1.15$)$ is consistent with (1.11)-(1.13). Alternatively, since $F_{4}=F_{1}^{(2)}, F^{\square}(x ; w)$ interpolates the limiting distributions of the second and the first eigenvalues of a random GOE matrix.

The meaning of $F^{\square}(x ; w)$ in terms of random matrices is not clear, but there is a Coulomb gas interpretation. In (7.64)-(7.65) of 7], the following density function is introduced. Suppose $2 N$ ordered particles on the positive real line, $0<\xi_{2 N}<\cdots<\xi_{2}<\xi_{1}$, are distributed according to the density function

$$
\frac{1}{Z_{2 N}} e^{A \sum_{j=1}^{2 N}(-1)^{j} \xi_{j}} \prod_{1 \leq i<j \leq 2 N}\left(\xi_{i}-\xi_{j}\right) \prod_{j=1}^{2 N} e^{-\xi_{j} / 2} d \xi_{j},
$$

where $Z_{2 N}$ is the normalization constant. Hence in addition to the usual Coulomb gas interaction, there is a additional attraction between neighboring pairs $\xi_{2 j-1}$ and $\xi_{2 j}, j=1, \cdots, N$. When $A=0$, this attraction vanishes, and one sees that (1.16) is the eigenvalue density for the $2 N \times 2 N$ Laguerre orthogonal ensemble (LOE). On the other hand, when $A \rightarrow \infty$, the neighboring particles $\xi_{2 j-1}, \xi_{2 j}, j=1,2, \cdots, N$, coalesce, and (1.16) becomes the eigenvalue density function for the $N \times N$ Laguerre symplectic ensemble (LSE). Thus (1.16) interpolates LOE and LSE eigenvalue distributions. This density function arises in a symmetric version of the growth model with a growth rule given by the exponential distribution considered in Proposition 1.4 of [31. A discrete version of the above density function was considered in (4.27) of [ \&], and the limiting distribution of the largest particle was precisely $F^{\square}(x ; w)$. Now formally taking the exponential limit (set $q=1-1 / L$ and $\alpha=1-A / L$ and take $L \rightarrow \infty$ ) of (4.27) of [8] (which is convincing, but is not justified yet), we see that if we set

$$
A=\frac{w}{N^{1 / 3}}
$$


in (1.16) and take $N \rightarrow \infty$, the scaled largest particle $\left(\xi_{1}-4 N\right) /\left(2 N^{1 / 3}\right)$ has the limiting distribution $F^{\square}(x ; w)$. We plan to exploit the justification of the exponential limit in a later publication.

On the other hand, the longest decreasing subsequence corresponds to the longest down/right path from $(0,1)$ to $(1,0)$ in the above point selection process. Thus it is clear that the distribution of $L_{n, m}^{\square}$ is insensitive to the number of fixed points. See Section 3 for results and discussions.

The other ensemble we consider is the set of signed involutions. A signed permutation $\pi$ is a bijection from $\{-n, \cdots,-1,1, \cdots, n\}$ onto itself which satisfies $\pi(x)=-\pi(-x)$. The limiting distribution for a random signed permutation is obtained by [47, 9]. In this paper we consider random signed involutions with/without constraint on fixed points and also negated points $(\pi(x)=-x)$. See Section 3 for results. Especially, we obtain another one-parameter family of distributions which now interpolates $F_{2}$ and $F_{1}^{2}$. Here $F_{1}^{2}$ has the meaning as the limiting distribution for the largest 'eigenvalue' of the super-imposition of the eigenvalues of two random GOE matrices. We note that in this case, $F_{2}$ is equal to the second largest 'eigenvalue' of such super-imposition (see the discussion at the end of Section 3).

For convenience of future reference, we summarize various definitions introduced above. By the $k^{\text {th }}$ row/column of $\pi$ we mean the $k^{\text {th }}$ row/column of the corresponding Young diagram under the Robinson-Schensted map.

Definition 1. Let $S_{n}$ be the symmetric group of $n$ letters and let $S_{n}^{\bigotimes}$ be the set of the bijections from $\{-n, \cdots,-2,-1,1,2, \cdots, n\}$ onto itself satisfying $\pi(x)=-\pi(-x)$. We define

$$
\begin{aligned}
& \tilde{S}_{n}=\left\{\pi \in S_{n}: \pi=\pi^{-1}\right\}, \\
& S_{n, m}=\left\{\pi \in \tilde{S}_{2 n+m}:|\{x: \pi(x)=x\}|=m\right\}, \\
& \tilde{S}_{n}^{\bigotimes}=\left\{\pi \in S_{n}^{\bigotimes}: \pi=\pi^{-1}\right\}, \\
& S_{n, m_{+}, m_{-}}^{\bigotimes}=\left\{\pi \in \tilde{S}_{2 n+m_{+}+m_{-}}^{\bigotimes}:|\{x: \pi(x)=x\}|=2 m_{+},|\{x: \pi(x)=-x\}|=2 m_{-}\right\}, \\
& \tilde{L}_{n}^{(k)}(\pi)=\text { the length of the } k^{t h} \text { row of } \pi \in \tilde{S}_{n}, \quad \tilde{L}_{n}=\tilde{L}_{n}^{(1)}, \\
& \tilde{L}_{n}^{\bigotimes,(k)}(\pi)=\text { the length of the } k^{t h} \text { row of } \pi \in \tilde{S}_{n}^{\bigotimes}, \quad \tilde{L}_{n}^{\bigotimes}=\tilde{L}_{n}^{\bigotimes,(1)}, \\
& L_{n, m}^{\square,(k)}(\pi)=\text { the length of the } k^{t h} \text { row of } \pi \in S_{n, m}^{\square}, \quad L_{n, m}^{\square}=L_{n, m}^{\square,(1)}, \\
& L_{n, m}^{\square,(k)}(\pi)=\text { the length of the } k^{t h} \text { column of } \pi \in S_{n, m}^{\square}, \quad L_{n, m}^{\square}=L_{n, m}^{\square,(1)}, \\
& L_{n, m_{+}, m_{-}}^{\bigotimes,(k)}(\pi)=\text { the length of the } k^{\text {th }} \text { row of } \pi \in S_{n, m_{+}, m_{-}}^{\bigotimes}, \quad L_{n, m_{+}, m_{-}}^{\bigotimes}=L_{n, m_{+}, m_{-}}^{\bigotimes,(1)} .
\end{aligned}
$$

The results of this paper were announced in [8]. Since we completed this paper, there have been two applications. One is to random vicious walker models [23, 8], and the other is to polynuclear growth models [42, 41, 6]. Indeed there are bijections between the above two applications and various ensembles considered in this paper, and thus the results in this paper can be employed to answer asymptotic questions in the above applications.

The proofs of our theorems use the Poissonization and de-Poissonization scheme of [30, 3]. We define the 
Poisson generating function, for example, for $L_{n, m}^{\square}$ by (see Definition 6)

$$
Q_{l}^{\square}\left(\lambda_{1}, \lambda_{2}\right):=e^{-\lambda_{1}-\lambda_{2}} \sum_{n_{1}, n_{2} \geq 0} \frac{\lambda_{1}^{n_{1}} \lambda_{2}^{n_{2}}}{n_{1} ! n_{2} !} \operatorname{Pr}\left(L_{n_{2}, n_{1}}^{\square} \leq l\right) .
$$

A generalization of the de-Poissonization lemma due to Johansson [30] yields that $\operatorname{Pr}\left(L_{n_{2}, n_{1}}^{\square} \leq l\right) \sim Q_{l}^{\square}\left(n_{1}, n_{2}\right)$ as $n_{1}, n_{2} \rightarrow \infty$; see Section 6 for the precise statement. Thus if we obtain the asymptotics of the generating function, the asymptotics of the coefficients will follow. The point of the scheme is that the Poisson generating functions can be expressed in terms of Toeplitz and/or Hankel determinants. The necessary algebraic work for this purpose was done in our earlier paper [7]. The general theory of orthogonal polynomials then tells us that Toeplitz/Hankel determinants can be expressed in terms of orthogonal polynomials. In the paper [7], it turned out that for all the ensembles being discussed in this paper, we need only one family of orthogonal polynomials : $\pi_{n}(z ; t)=z^{n}+\cdots$ which is orthogonal with respect to the weight $e^{t \cos \theta} d \theta /(2 \pi)$ on the unit circle. This orthogonal polynomial is precisely the same orthogonal polynomial used in [3] to analyze the random permutation problem. The authors in [3] computed the uniform asymptotics of the normalization constant $N_{n}(t)$ of $\pi_{n}$ as $n, t \rightarrow \infty$ using the steepest-descent analysis for the corresponding Riemann-Hilbert problem (see (5.3) below). The difference between the present paper and [3] is that we need $\pi_{n}(-\alpha ; t)$ for all $\alpha \geq 0$, in contrast to [3] where only one quantity, $N_{n}(t)$, is needed. But in order to analyze $N_{n}(t)$, [3] controlled in a uniform way the asymptotic behavior of the solution to the associated Riemann-Hilbert problem. Therefore the asymptotics of $\pi_{n}(-\alpha ; t)$ for $\alpha$ uniformly apart from 1 can be (almost) directly read off from the analysis of [3], which eventually imply (1.11). The point $z=-1(\alpha=1)$ in the complex plane plays a special role in this Riemann-Hilbert analysis as discussed in [3] - it is the point where a gap starts to open up in the support of the associated equilibrium measure as the relation of $t$ to $n$ varies. When $\alpha \rightarrow 1$ as $n \rightarrow \infty$ according to the scaling (1.14) (which is required for (1.15)), we need more careful analysis of the Riemann-Hilbert problem which is the new part of the asymptotic analysis of the orthogonal polynomials and the Riemann-Hilbert problem. In this paper we establish this goal by extending the analysis of [3]. In the analysis of the Riemann-Hilbert problem in Section 10, we give a rather sketchy presentation for the parts which overlap the work of [3], but we give a full proof for the new analysis required for the case $\alpha \rightarrow 1$. We also rework portions of [3] as necessary for consistency of presentation.

This paper is organized as follows. Section 2 defines the Tracy-Widom distribution functions as well as new classes of distribution functions which will be used to state the main results, and discusses their properties (Lemma 2.1). The main results of the paper are then stated in Section 3 . Determinantal formulae and orthogonal polynomial expressions for the Poisson generating functions are taken from [7] and summarized in Section 4. In Section 5 , we state the main estimates of the relevant quantities of orthogonal polynomials; these estimates are key to the proofs of the theorems of Section 3. The de-Poissonization lemmas are stated in Section 6. Proofs of the main theorems are given in Section 7 for involutions with a constraint on the number of fixed points (Theorems 3.1, 3.2, 3.3 and 3.5), and in Section 8 for general involutions and equivalently for $M_{n}^{1}$ (Theorems 3.4 and 3.6), respectively. The case when $\alpha>1$ is considered in Section 9 (see Remark to Theorem 3.3). Finally the Riemann-Hilbert analysis is given in Section 10, which proves the propositions in Section 5 . 
Notational remarks. The ensemble $\tilde{S}_{n}$ in the present paper is identical to $\tilde{S}_{n}^{\square}$ in []]. In [7], $\tilde{S}_{n}^{\nabla}$ is introduced to denote the ensemble of neginvolutions, and the authors investigated the longest increasing subsequence of $\pi \in \tilde{S}_{n}^{\square}$. But there is a bijection between $\tilde{S}_{n}^{\triangleright}$ and $\tilde{S}_{n}^{\square}$ and the longest increasing subsequence of $\pi \in \tilde{S}_{n}^{\triangleright}$ corresponds to the longest decreasing subsequence of the image of $\pi$. In the present paper, we choose the view point of considering both the increasing and decreasing subsequences of involutions of the same ensemble rather than considering only the increasing subsequences of involutions of the different ensembles.

Acknowledgments. The authors would like to thank Percy Deift for helpful discussions and encouragement, especially for his help in proving Lemma 2.1. We would also like to acknowledge many useful conversations and communications with Peter Forrester, Kurt Johansson, Charles Newman and Harold Widom. Special thanks is due to the referee who gave us crucial advice, improving the exposition of the paper significantly. The work of the first author was supported in part by a Sloan Doctoral Dissertation Fellowship during the academic year 1998-1999 when he was a graduate student at Courant Institute of Mathematical Sciences.

\section{Limiting distribution functions}

Let $u(x)$ be the solution of the Painlevé II (PII) equation,

$$
u_{x x}=2 u^{3}+x u
$$

with the boundary condition

$$
u(x) \sim-A i(x) \text { as } \quad x \rightarrow+\infty,
$$

where $A i$ is the Airy function. The proof of the (global) existence and the uniqueness of the solution was first established in 27]: the asymptotics as $x \rightarrow \pm \infty$ are (see e.g. [27, 19])

$$
\begin{aligned}
& u(x)=-A i(x)+O\left(\frac{e^{-(4 / 3) x^{3 / 2}}}{x^{1 / 4}}\right), \quad \text { as } x \rightarrow+\infty, \\
& u(x)=-\sqrt{\frac{-x}{2}}\left(1+O\left(\frac{1}{x^{2}}\right)\right), \quad \text { as } x \rightarrow-\infty .
\end{aligned}
$$

Recall that $A i(x) \sim \frac{e^{-(2 / 3) x^{3 / 2}}}{2 \sqrt{\pi} x^{1 / 4}}$ as $x \rightarrow+\infty$. Define

$$
v(x):=\int_{\infty}^{x}(u(s))^{2} d s
$$

so that $v^{\prime}(x)=(u(x))^{2}$.

We can now introduce the Tracy-Widom (TW) distributions. (Note that $q:=-u$, which Tracy and Widom used in their papers, solves the same differential equation with the boundary condition $q(x) \sim+A i(x)$ as $x \rightarrow \infty$.) 
Definition 2 (TW distribution functions). Set

$$
\begin{aligned}
& F(x):=\exp \left(\frac{1}{2} \int_{x}^{\infty} v(s) d s\right)=\exp \left(-\frac{1}{2} \int_{x}^{\infty}(s-x)(u(s))^{2} d s\right) \\
& E(x):=\exp \left(\frac{1}{2} \int_{x}^{\infty} u(s) d s\right)
\end{aligned}
$$

and set

$$
\begin{aligned}
& F_{2}(x):=\quad F(x)^{2} \quad=\exp \left(-\int_{x}^{\infty}(s-x)(u(s))^{2} d s\right), \\
& F_{1}(x):=\quad F(x) E(x) \quad=\left(F_{2}(x)\right)^{1 / 2} e^{\frac{1}{2} \int_{x}^{\infty} u(s) d s}, \\
& F_{4}(x):=F(x)\left[E(x)^{-1}+E(x)\right] / 2=\left(F_{2}(x)\right)^{1 / 2}\left[e^{-\frac{1}{2} \int_{x}^{\infty} u(s) d s}+e^{\frac{1}{2} \int_{x}^{\infty} u(s) d s}\right] / 2 .
\end{aligned}
$$

In [45] and [46], Tracy and Widom proved that under proper centering and scaling, the distribution of the largest eigenvalue of a random GUE/GOE/GSE matrix converges to $F_{2}(x) / F_{1}(x) / F_{4}(x)$ as the size of the matrix becomes large. We note that from the asymptotics (2.3) and (2.4), for some positive constant $c$,

$$
\begin{array}{ll}
F(x)=1+O\left(e^{-c x^{3 / 2}}\right) & \text { as } x \rightarrow+\infty, \\
E(x)=1+O\left(e^{-c x^{3 / 2}}\right) & \text { as } x \rightarrow+\infty, \\
F(x)=O\left(e^{-c|x|^{3}}\right) & \text { as } x \rightarrow-\infty, \\
E(x)=O\left(e^{-c|x|^{3 / 2}}\right) & \text { as } x \rightarrow-\infty .
\end{array}
$$

Hence in particular, $\lim _{x \rightarrow+\infty} F_{\beta}(x)=1$ and $\lim _{x \rightarrow-\infty} F_{\beta}(x)=0, \beta=1,2,4$. Monotonicity of $F_{\beta}(x)$ follows from the fact that $F_{\beta}(x)$ is the limit of a sequence of distribution functions. Therefore $F_{\beta}(x)$ is indeed a distribution function.

Definition 3. Define $\chi_{\mathrm{GOE}}, \chi_{\mathrm{GUE}}$ and $\chi_{\mathrm{GSE}}$ to be random variables whose distribution functions are given by $F_{1}(x), F_{2}(x)$ and $F_{4}(x)$, respectively. Define $\chi_{\mathrm{GOE}^{2}}$ to be a random variable with the distribution function $F_{1}(x)^{2}$.

As indicated in Introduction, we need new classes of distribution functions to describe the transitions from $\chi_{\mathrm{GSE}}$ to $\chi_{\mathrm{GOE}}$ and from $\chi_{\mathrm{GUE}}$ to $\chi_{\mathrm{GOE}^{2}}$. First we consider the Riemann-Hilbert problem (RHP) for the Painlevé II equation [20, 29]. Let $\Gamma$ be the real line $\mathbb{R}$, oriented from $+\infty$ to $-\infty$, and let $m(\cdot ; x)$ be the solution of the following RHP:

$$
\left\{\begin{array}{l}
m(z ; x) \quad \text { is analytic in } z \in \mathbb{C} \backslash \Gamma, \\
m_{+}(z ; x)=m_{-}(z ; x)\left(\begin{array}{cc}
1 & -e^{-2 i\left(\frac{4}{3} z^{3}+x z\right)} \\
e^{2 i\left(\frac{4}{3} z^{3}+x z\right)} & 0
\end{array}\right) \quad \text { for } z \in \Gamma, \\
m(z ; x)=I+O\left(\frac{1}{z}\right) \quad \text { as } z \rightarrow \infty .
\end{array}\right.
$$

Here $m_{+}(z ; x)$ (resp,. $\left.m_{-}\right)$is the limit of $m\left(z^{\prime} ; x\right)$ as $z^{\prime} \rightarrow z$ from the left (resp., right) of the contour $\Gamma$ : $m_{ \pm}(z ; x)=\lim _{\epsilon \downarrow 0} m(z \mp i \epsilon ; x)$. Relation (2.15) corresponds to the RHP for the PII equation with the special 
monodromy data $p=-q=1, r=0$ (see [20, 29], also [22, 19]). In particular if the solution is expanded at $z=\infty$,

$$
m(z ; x)=I+\frac{m_{1}(x)}{z}+O\left(\frac{1}{z^{2}}\right), \quad \text { as } z \rightarrow \infty
$$

we have

$$
\begin{aligned}
& 2 i\left(m_{1}(x)\right)_{12}=-2 i\left(m_{1}(x)\right)_{21}=u(x), \\
& 2 i\left(m_{1}(x)\right)_{22}=-2 i\left(m_{1}(x)\right)_{11}=v(x),
\end{aligned}
$$

where $u(x)$ and $v(x)$ are defined in (2.1)-(2.5).

Now we define two one-parameter families of distribution functions.

Definition 4. Let $m(z ; x)$ be the solution of RHP (2.15) and denote by $m_{j k}(z ; x)$ the $(j k)$-entry of $m(z ; x)$. For $w>0$, define

$$
F^{\square}(x ; w):=F(x)\left\{\left[m_{22}(-i w ; x)-m_{12}(-i w ; x)\right] E(x)^{-1}+\left[m_{22}(-i w ; x)+m_{12}(-i w ; x)\right] E(x)\right\} / 2,
$$

and for $w<0$, define

$$
\begin{aligned}
F^{\square}(x ; w) & :=e^{\frac{8}{3} w^{3}-2 x w} F(x) \\
& \times\left\{\left[-m_{21}(-i w ; x)+m_{11}(-i w ; x)\right] E(x)^{-1}-\left[m_{21}(-i w ; x)+m_{11}(-i w ; x)\right] E(x)\right\} / 2 .
\end{aligned}
$$

Also define

$$
\begin{array}{lll}
F^{\bigotimes}(x ; w) & :=m_{22}(-i w ; x) F_{2}(x), & w>0, \\
F^{\bigotimes}(x ; w) & :=\quad-e^{\frac{8}{3} w^{3}-2 x w} m_{21}(-i w ; x) F_{2}(x), & w<0 .
\end{array}
$$

First, $F^{\square}(x ; w)$ and $F^{\square}(x ; w)$ are real from Lemma 2.1 (i) below. Note that $F^{\square}(x ; w)$ and $F^{\square}(x ; w)$ are continuous at $w=0$ since at $z=0$, the jump condition of the RHP (2.15) implies $\left(m_{12}\right)_{+}(0 ; x)=-\left(m_{11}\right)_{-}(0 ; x)$ and $\left(m_{22}\right)_{+}(0 ; x)=-\left(m_{21}\right)_{-}(0 ; x)$. In fact, $F^{\square}(x ; w)$ and $F^{\bigotimes}(x ; w)$ are entire in $w \in \mathbb{C}$ from the RHP (2.15).

From (2.11)-(2.14) and (2.24)-(2.27) below, we see that

$$
\lim _{x \rightarrow+\infty} F^{\square}(x ; w), F^{\bigotimes}(x ; w)=1, \quad \lim _{x \rightarrow-\infty} F^{\square}(x ; w), F^{\bigotimes}(x ; w)=0
$$

for any fixed $w \in \mathbb{R}$. Also Theorem 3.2 below shows that $F^{\square}(x ; w)$ and $F^{\bigotimes}(x ; w)$ are limits of distribution functions, implying that they are monotone in $x$. Therefore, $F^{\square}(x ; w)$ and $F^{\square}(x ; w)$ are indeed distribution functions for each $w \in \mathbb{R}$.

Definition 5. Define $\chi_{w}^{\square}$ and $\chi_{w}^{\bigotimes}$ to be random variables with distribution functions $F^{\square}(x ; w)$ and $F^{\bigotimes}(x ; w)$, respectively.

We close this section by summarizing some properties of $m(-i w ; x)$ in the following lemma. In particular the lemma implies that $F^{\square}(x ; w)$ interpolates between $F_{4}(x)$ and $F_{1}(x)$, and $F^{\bigotimes}(x ; w)$ interpolates between $F_{2}(x)$ and $F_{1}(x)^{2}$ (see Corollary 2.2). 
Lemma 2.1. Let $\sigma_{3}=\left(\begin{array}{cc}1 & 0 \\ 0 & -1\end{array}\right), \sigma_{1}=\left(\begin{array}{ll}0 & 1 \\ 1 & 0\end{array}\right)$, and set $[a, b]=a b-b a$.

(i). For real $w, m(-i w ; x)$ is real.

(ii). For fixed $w \in \mathbb{R}$, we have

$$
\begin{aligned}
& m(-i w ; x)=\left(I+\left(e^{-c x^{3 / 2}}\right)\right)\left(\begin{array}{cc}
1 & -e^{\frac{8}{3} w^{3}-2 x w} \\
0 & 1
\end{array}\right), \quad w>0, x \rightarrow+\infty, \\
& m(-i w ; x)=\left(I+\left(e^{-c x^{3 / 2}}\right)\right)\left(\begin{array}{cc}
1 & 0 \\
-e^{-\frac{8}{3} w^{3}+2 x w} & 1
\end{array}\right), \quad w<0, x \rightarrow+\infty \\
& m(-i w ; x) \sim \frac{1}{\sqrt{2}}\left(\begin{array}{cc}
1 & -1 \\
1 & 1
\end{array}\right) e^{\left(-\frac{4}{3} w^{3}+x w\right) \sigma_{3}} e^{\left(\frac{\sqrt{2}}{3}(-x)^{3 / 2}+\sqrt{2} w^{2}(-x)^{1 / 2}\right) \sigma_{3}}, \quad w>0, x \rightarrow-\infty, \\
& m(-i w ; x) \sim \frac{1}{\sqrt{2}}\left(\begin{array}{cc}
1 & 1 \\
-1 & 1
\end{array}\right) e^{\left(-\frac{4}{3} w^{3}+x w\right) \sigma_{3}} e^{\left(-\frac{\sqrt{2}}{3}(-x)^{3 / 2}-\sqrt{2} w^{2}(-x)^{1 / 2}\right) \sigma_{3}}, \quad w<0, x \rightarrow-\infty .
\end{aligned}
$$

(iii). For any $x$, we have

$$
\lim _{w \rightarrow 0^{+}} m(-i w ; x)=\lim _{w \rightarrow 0^{-}} \sigma_{1} m(-i w ; x) \sigma_{1}=\left(\begin{array}{cc}
\frac{1}{2}\left(E(x)^{2}+E(x)^{-2}\right) & -E(x)^{2} \\
\frac{1}{2}\left(-E(x)^{2}+E(x)^{-2}\right) & E(x)^{2}
\end{array}\right) .
$$

(iv). For fixed $w \in \mathbb{R} \backslash\{0\}, m(-i w ; x)$ solves the differential equation

$$
\frac{d}{d x} m=w\left[m, \sigma_{3}\right]+u(x) \sigma_{1} m,
$$

where $u(x)$ is the solution of the PII equation (2.1), (2.2).

(v). For fixed $x, m(-i w ; x)$ solves

$$
\frac{\partial}{\partial w} m=\left(-4 w^{2}+x\right)\left[m, \sigma_{3}\right]-4 w u(x) \sigma_{1} m-2\left(\begin{array}{cc}
u^{2} & -u^{\prime} \\
u^{\prime} & -u^{2}
\end{array}\right) m .
$$

(vi). For any $x$, we have

$$
m(z ; x)=\sigma_{1} m(-z ; x) \sigma_{1} .
$$

Corollary 2.2. We have

$$
\begin{aligned}
F^{\square}(x ; 0) & =F_{1}(x), \\
\lim _{w \rightarrow \infty} F^{\square}(x ; w) & =F_{4}(x), \\
\lim _{w \rightarrow-\infty} F^{\square}(x ; w) & =0, \\
F^{\square}(x ; 0) & =F_{1}(x)^{2}, \\
\lim _{w \rightarrow \infty} F^{\square}(x ; w) & =F_{2}(x), \\
\lim _{w \rightarrow-\infty} F^{\square}(x ; w) & =0 .
\end{aligned}
$$


Proof. The values at $w=0$ follow from (2.28). For $w \rightarrow \pm \infty$, note from the RHP (2.15) that we have $\lim _{z \rightarrow \infty} m(z ; x)=I$.

Proof of Lemma 2.1. Let $v(z)=v(z ; x)$ denote the jump matrix of the RHP (2.15). Since $\overline{v(-z)}=v(z)$ for $z \in \mathbb{R}, M(z):=\overline{m(-\bar{z} ; x)}$ also solves the same RHP. By the uniqueness of the solution of the RHP (2.15), we have

$$
\overline{m(-\bar{z} ; x)}=m(z ; x), \quad z \in \mathbb{C} \backslash \mathbb{R} .
$$

Thus, $m(-i w ; x)$ is real for $w \in \mathbb{R}$, thus proving (i).

By the symmetry of the jump matrix, $\sigma_{1} v(-z)^{-1} \sigma_{1}=v(z)$, we obtain, by a argument similar to (i),

$$
\sigma_{1} m(-z ; x) \sigma_{1}=m(z ; x)
$$

which is (vi).

The asymptotics results (ii) as $x \rightarrow \pm \infty$ follow from the calculations in Section 6, pp.329-333, of [19].

For the proof of (iv), define a new matrix function

$$
f(z ; x):=m(z ; x) e^{-i \theta \sigma_{3}}, \quad \theta:=\frac{4}{3} z^{3}+x z .
$$

Then $f(\cdot ; x)$ satisfies the jump condition $f_{+}(z ; x)=f_{-}(z ; x)\left(\begin{array}{cc}1 & -1 \\ 1 & 0\end{array}\right)$ for $z \in \mathbb{R}$, and $f(z ; x) e^{i \theta \sigma_{3}} \rightarrow I$ as $z \rightarrow \infty$. Since the jump matrix for $f(z ; x)$ is independent of $x, f^{\prime}(z ; x)$, the derivative with respect to $x$, satisfies $f_{+}^{\prime}(z ; x)=f_{-}^{\prime}(z ; x)\left(\begin{array}{cc}1 & -1 \\ 1 & 0\end{array}\right)$, and $f^{\prime} e^{i \theta \sigma_{3}}+i \theta^{\prime} f \sigma_{3} e^{i \theta \sigma_{3}} \rightarrow 0$ as $z \rightarrow \infty$. Hence $f^{\prime} f^{-1}$ has no jump across $\mathbb{R}$, and satisfies $f^{\prime} f^{-1}+i \theta^{\prime} f \sigma_{3} f^{-1} \rightarrow 0$ as $z \rightarrow \infty$. If we write $m(z ; x)=I+\frac{m_{1}(x)}{z}+O\left(1 / z^{2}\right)$ as $z \rightarrow \infty$, we have $i \theta^{\prime} f \sigma_{3} f^{-1}=i z \sigma_{3}+i\left[m_{1}, \sigma_{3}\right]+O\left(z^{-1}\right)$ as $z \rightarrow \infty$. Thus $f^{\prime} f^{-1}$ is entire and as $z \rightarrow \infty, f^{\prime} f^{-1} \sim-i z \sigma_{3}-i\left[m_{1}, \sigma_{3}\right]$. Therefore by Liouville's theorem, we obtain

$$
f^{\prime}(z ; x)(f(z ; x))^{-1}=-i z \sigma_{3}-i\left[m_{1}, \sigma_{3}\right] .
$$

Recalling that $u(x)=2 i\left(m_{1}(x)\right)_{12}=-2 i\left(m_{1}(x)\right)_{21}$ in 2.17$)$, we have $\left[m_{1}, \sigma_{3}\right]=i u(x) \sigma_{1}$. Changing $f$ to $m$ from 2.40$),(2.41)$ is

$$
\frac{d}{d x} m(z ; x)=i z\left[m(z ; x), \sigma_{3}\right]+u(x) \sigma_{1} m(z ; x)
$$

This is (2.29) when $z=-i w$.

The proof of (v) is very similar to that of (iv), and the detail is left to the reader. We only note that in the derivation of (v), we need the identity

$$
\frac{d}{d x} m_{1}=i\left[m_{2}, \sigma_{3}\right]-i\left[m_{1}, \sigma_{3}\right] m_{1}
$$

which can be obtained from (2.42) by setting $m(z ; x)=I+\frac{m_{1}(x)}{z}+\frac{m_{2}(x)}{z^{2}}+O\left(1 / z^{3}\right)$ as $z \rightarrow \infty$.

Finally we prove (iii). Note that $\lim _{w \rightarrow 0^{ \pm}} m(-i w ; x)=m_{ \pm}(0 ; x)$. From the jump condition at $z=0$, we have

$$
m_{+}(0 ; x)=m_{-}(0 ; x)\left(\begin{array}{cc}
1 & -1 \\
1 & 0
\end{array}\right)
$$


Letting $z \rightarrow 0, \operatorname{Im} z>0$, in (vi), we have $\sigma_{1} m_{+}(0 ; x) \sigma_{1}=m_{-}(0 ; x)$, which together with (2.44) implies that $m_{+}(0 ; x)=\sigma_{1} m_{+}(0 ; x) \sigma_{1}\left(\begin{array}{cc}1 & -1 \\ 1 & 0\end{array}\right)$. Thus we have

$$
m_{+}(0 ; x)=\left(\begin{array}{cc}
a(x) & b(x) \\
a(x)+b(x) & -b(x)
\end{array}\right)
$$

for some $a(x), b(x)$. Also the condition $\operatorname{det} v(z)=1$ for all $z \in \mathbb{R}$ implies that $\operatorname{det} m(z ; x)=1$ for all $z \in \mathbb{C} \backslash \mathbb{R}$, and hence we have

$$
b^{2}+2 a b+1=0 .
$$

Now letting $z \rightarrow 0, \operatorname{Im} z<0$ in (2.42), we obtain

$$
m_{+}^{\prime}(0 ; x)\left(m_{+}(0 ; x)\right)^{-1}=\left(\begin{array}{cc}
0 & u(x) \\
u(x) & 0
\end{array}\right) .
$$

Thus from (2.45) and (2.46), $b^{\prime} / b=-u$, which has the solution

$$
b(x)=b(y) e^{-\int_{y}^{x} u(s) d s} .
$$

From (2.24) with $w=0^{+}$, we have

$$
b(x)=\left(m_{12}\right)_{+}(0 ; x) \rightarrow-1, \quad \text { as } x \rightarrow+\infty .
$$

Therefore, $b(x)=-e^{\int_{x}^{\infty} u(s) d s}$, which is $-E(x)^{2}$ from (2.7). Now 2.46) gives $a(x)=\frac{1}{2}\left(E(x)^{2}+E(x)^{-2}\right)$, proving (2.28).

\section{Statement of results}

\subsection{Involutions with constraint on the number of fixed points}

Recall (Definition 1 in Introduction) the ensembles $\tilde{S}_{n, m}, \tilde{S}_{n, m_{+}, m_{-}}^{\bowtie}$ of (signed) involutions with constraint on the number of fixed (and negated) points. We scale the random variables:

$$
\begin{aligned}
\chi_{n, m}^{\square} & :=\frac{L_{n, m}^{\square}-2 \sqrt{2 n+m}}{(2 n+m)^{1 / 6}}, \\
\chi_{n, m}^{\square} & :=\frac{L_{n, m}^{\square}-2 \sqrt{2 n+m}}{(2 n+m)^{1 / 6}}, \\
\chi_{n, m_{+}, m_{-}}^{\bigotimes} & :=\frac{L_{n, m_{+}, m_{-}}^{\square}-2 \sqrt{4 n+2 m_{+}+2 m_{-}}}{2^{2 / 3}\left(4 n+2 m_{+}+2 m_{-}\right)^{1 / 6}} .
\end{aligned}
$$

Theorem 3.1. For fixed $\alpha$ and $\beta$, we have:

$$
\begin{aligned}
& \lim _{n \rightarrow \infty} \operatorname{Pr}\left(\chi_{n,[\sqrt{2 n} \alpha]}^{\square} \leq x\right)=F_{4}(x), \quad 0 \leq \alpha<1, \\
& \lim _{n \rightarrow \infty} \operatorname{Pr}\left(\chi_{n,[\sqrt{2 n}]}^{\square} \leq x\right)=F_{1}(x), \\
& \lim _{n \rightarrow \infty} \operatorname{Pr}\left(\chi_{n,[\sqrt{2 n} \alpha]}^{\square} \leq x\right)=0, \quad \alpha>1 .
\end{aligned}
$$




$$
\lim _{n \rightarrow \infty} \operatorname{Pr}\left(\chi_{n,[\sqrt{2 n} \beta]}^{\triangleright} \leq x\right)=F_{1}(x), \quad \beta \geq 0 .
$$

$$
\begin{aligned}
& \lim _{n \rightarrow \infty} \operatorname{Pr}\left(\chi_{n,[\sqrt{n} \alpha],[\sqrt{n} \beta]}^{\bigotimes} \leq x\right)=F_{2}(x), \quad 0 \leq \alpha<1, \beta \geq 0, \\
& \lim _{n \rightarrow \infty} \operatorname{Pr}\left(\chi_{n,[\sqrt{n}],[\sqrt{n} \beta]}^{\otimes} \leq x\right)=F_{1}(x)^{2}, \quad \beta \geq 0, \\
& \lim _{n \rightarrow \infty} \operatorname{Pr}\left(\chi_{n,[\sqrt{n} \alpha],[\sqrt{n} \beta]}^{\otimes} \leq x\right)=0, \quad \alpha>1, \beta \geq 0 .
\end{aligned}
$$

As indicated earlier in the introduction, as $\alpha \rightarrow 1$ at a certain rate, we see smooth transitions.

Theorem 3.2. For fixed $w \in \mathbb{R}$ and $\beta \geq 0$, we have

$$
\begin{aligned}
\lim _{n \rightarrow \infty} \operatorname{Pr}\left(\chi_{n, m}^{\square} \leq x\right) & =F^{\square}(x ; w), \quad m=\left[\sqrt{2 n}-2 w(2 n)^{1 / 3}\right], \\
\lim _{n \rightarrow \infty} \operatorname{Pr}\left(\chi_{n, m_{+}, m_{-}}^{\square} \leq x\right) & =F^{\bigotimes}(x ; w), \quad m_{+}=\left[\sqrt{n}-2 w n^{1 / 3}\right], m_{-}=[\sqrt{n} \beta] .
\end{aligned}
$$

From Corollary 2.2, this result is consistent with Theorem 3.1. We also have convergence of moments.

Theorem 3.3. For any $p=1,2,3, \cdots$, the followings hold. For fixed $\alpha$ and $\beta$,

$$
\begin{aligned}
& \lim _{n \rightarrow \infty} \mathrm{E}\left(\left(\chi_{n,[\sqrt{2 n} \alpha]}^{\square}\right)^{p}\right)=\mathrm{E}\left(\left(\chi_{G S E}\right)^{p}\right), \quad 0 \leq \alpha<1, \\
& \lim _{n \rightarrow \infty} \mathrm{E}\left(\left(\chi_{n,[\sqrt{2 n}]}^{\square}\right)^{p}\right)=\mathrm{E}\left(\left(\chi_{G O E}\right)^{p}\right), \\
& \lim _{n \rightarrow \infty} \mathrm{E}\left(\left(\chi_{n,[\sqrt{2 n} \beta]}^{\square}\right)^{p}\right)=\mathrm{E}\left(\left(\chi_{G O E}\right)^{p}\right), \quad 0 \leq \beta, \\
& \lim _{n \rightarrow \infty} \mathrm{E}\left(\left(\chi_{n,[\sqrt{n} \alpha],[\sqrt{n} \beta]}^{\otimes}\right)^{p}\right)=\mathrm{E}\left(\left(\chi_{G U E}\right)^{p}\right), \quad 0 \leq \alpha<1, \beta \geq 0, \\
& \lim _{n \rightarrow \infty} \mathrm{E}\left(\left(\chi_{n,[\sqrt{n}],[\sqrt{n} \beta]}^{\otimes}\right)^{p}\right)=\mathrm{E}\left(\left(\chi_{G O E^{2}}\right)^{p}\right), \quad \beta \geq 0 .
\end{aligned}
$$

Also for fixed $w \in \mathbb{R}$ and $\beta \geq 0$,

$$
\begin{aligned}
\lim _{n \rightarrow \infty} \mathrm{E}\left(\left(\chi_{n, m}^{\square}\right)^{p}\right) & =\mathrm{E}\left(\left(\chi_{w}^{\square}\right)^{p}\right), & m=\left[\sqrt{2 n}-2 w(2 n)^{1 / 3}\right], \\
\lim _{n \rightarrow \infty} \mathrm{E}\left(\left(\chi_{n, m_{+}, m_{-}}^{\bigotimes}\right)^{p}\right) & =\mathrm{E}\left(\left(\chi_{w}^{\bigotimes}\right)^{p}\right), & m_{+}=\left[\sqrt{n}-2 w n^{1 / 3}\right], m_{-}=[\sqrt{n} \beta] .
\end{aligned}
$$

Remark. Theorem 3.1 shows that when $\alpha>1$ is fixed, we have used incorrect scaling. When properly scaled, the resulting limiting distribution is Gaussian. See Section 9 for the statement and the proof.

The proof of Theorem 3.1, 3.2, 3.3 are provided in Section 7 .

In terms of the points selection process, which is a version of (directed site) percolation, mentioned in the Introduction, the above results show that the limiting distribution of the longest path depends on the geometry of the domain, while the order of fluctuation is the same: $(\text { mean })^{1 / 3}$. From (1.4), the longest up/right path in a rectangle $0 \leq x, y \leq 1$ has $F_{2}$ in the limit, while the longest up/right path in a lower triangle $0 \leq x<y \leq 1$ has $F_{4},(3.4)$, in the limit if there is no points on the edge $0 \leq x=y \leq 1$. If there are points on $0 \leq x=y \leq 1$, they 
affect the length of the longest up/right path. On the other hand, the longest down/right path corresponding to $L_{n, m}^{\square}$ can be thought as the longest path from the point $(0,1)$ to the line $0 \leq x=y \leq 1$. Thus the result (3.7) shows that the point-to-line maximizing path has different limiting distribution from the point-to-point maximizing path, $F_{2}$ from (1.4), though the fluctuation order is identical. One can also state similar results for Poisson process (see Theorem 7.3 below) and also certain directed site percolation processes considered by 31 (see [8]). This observation came from discussions of one of us (J.B.) with Charles Newman to whom we are specially grateful.

\subsection{General involutions}

Now we consider general involutions and signed involutions without any conditions on the number of fixed or negated points.

Theorem 3.4. For any fixed $x \in \mathbb{R}$, we have

$$
\begin{gathered}
\lim _{n \rightarrow \infty} \operatorname{Pr}\left(\tilde{\chi}_{n}:=\frac{\tilde{L}_{n}-2 \sqrt{n}}{n^{1 / 6}} \leq x\right)=F_{1}(x), \\
\lim _{n \rightarrow \infty} \operatorname{Pr}\left(\tilde{\chi}_{n}^{\bigotimes}:=\frac{\tilde{L}_{n}^{\bigotimes}-2 \sqrt{2 n}}{2^{2 / 3}(2 n)^{1 / 6}} \leq x\right)=F_{1}(x)^{2} .
\end{gathered}
$$

Also for any $p=1,2,3, \cdots$,

$$
\begin{aligned}
& \lim _{n \rightarrow \infty} \mathrm{E}\left(\left(\tilde{\chi}_{n}\right)^{p}\right)=\mathrm{E}\left(\left(\chi_{G O E}\right)^{p}\right), \\
& \lim _{n \rightarrow \infty} \mathrm{E}\left(\left(\tilde{\chi}_{n}^{\bigotimes}\right)^{p}\right)=\mathrm{E}\left(\left(\chi_{G O E^{2}}\right)^{p}\right) .
\end{aligned}
$$

As mentioned in the introduction, this result proves that the first row of a random Young diagram under the 1-Plancherel measure $M_{n}^{1}$ behaves statistically like the largest eigenvalue of a random GOE matrix as $n \rightarrow \infty$. The proof of the theorem is given in Section 8 .

\subsection{Second rows}

For the second row, we scale the same way as in (3.1)-(3.3), and denote the scaled random variables by $\chi_{n, m}^{\square,(2)}$, $\chi_{n, m}^{\nabla,(2)}$ and $\chi_{n, m_{+}, m_{-}}^{\otimes,(2)}$, respectively.

Theorem 3.5. Let $\alpha, \beta \geq 0$ be fixed. Then

$$
\begin{aligned}
\lim _{n \rightarrow \infty} \operatorname{Pr}\left(\chi_{n, \sqrt{2 n} \alpha}^{\square,(2)}\right) & =F_{4}(x), \\
\lim _{n \rightarrow \infty} \operatorname{Pr}\left(\chi_{n,[\sqrt{2 n} \beta]}^{\unrhd,(2)}\right) & =F_{4}(x), \\
\lim _{n \rightarrow \infty} \operatorname{Pr}\left(\chi_{n,[\sqrt{n} \alpha],[\sqrt{n} \beta]}^{\bigotimes,(2)}\right) & =F_{2}(x),
\end{aligned}
$$


and for any $p=1,2,3, \cdots$,

$$
\begin{aligned}
\lim _{n \rightarrow \infty} \mathrm{E}\left(\left(\chi_{n, \sqrt{2 n} \alpha}^{\square,(2)}\right)^{p}\right) & =\mathrm{E}\left(\left(\chi_{G S E}\right)^{p}\right), \\
\lim _{n \rightarrow \infty} \mathrm{E}\left(\left(\chi_{n,[\sqrt{2 n} \beta]}^{\square,(2)}\right)^{p}\right) & =\mathrm{E}\left(\left(\chi_{G S E}\right)^{p}\right), \\
\lim _{n \rightarrow \infty} \mathrm{E}\left(\left(\chi_{n,[\sqrt{n} \alpha],[\sqrt{n} \beta]}^{\bigotimes,(2)}\right)^{p}\right) & =\mathrm{E}\left(\left(\chi_{G U E}\right)^{p}\right) .
\end{aligned}
$$

This theorem is proved in Section 7 .

As in the first row, these results yield the following theorem on the second rows of general (signed) involutions. The proof is very similar to the proof of Theorem 3.4 and we skip the details.

Theorem 3.6. For any fixed $x \in \mathbb{R}$, we have

$$
\begin{array}{r}
\lim _{n \rightarrow \infty} \operatorname{Pr}\left(\tilde{\chi}_{n}^{(2)}:=\frac{\tilde{L}_{n}^{(2)}-2 \sqrt{n}}{n^{1 / 6}} \leq x\right)=F_{4}(x), \\
\lim _{n \rightarrow \infty} \operatorname{Pr}\left(\tilde{\chi}_{n}^{\bigotimes,(2)}:=\frac{\tilde{L}_{n}^{\bigotimes,(2)}-2 \sqrt{2 n}}{2^{2 / 3}(2 n)^{1 / 6}} \leq x\right)=F_{2}(x) .
\end{array}
$$

Also for any $p=1,2,3, \cdots$,

$$
\begin{aligned}
\lim _{n \rightarrow \infty} \mathrm{E}\left(\left(\tilde{\chi}_{n}^{(2)}\right)^{p}\right) & =\mathrm{E}\left(\left(\chi_{G S E}\right)^{p}\right), \\
\lim _{n \rightarrow \infty} \mathrm{E}\left(\left(\tilde{\chi}_{n}^{\bigotimes,(2)}\right)^{p}\right) & =\mathrm{E}\left(\left(\chi_{G U E}\right)^{p}\right) .
\end{aligned}
$$

We conclude this section with some remarks on GOE and GSE. If the conjecture given in the introduction that the $k^{\text {th }}$ row of a random involution behaves in the limit like the $k^{\text {th }}$ largest eigenvalue of a random GOE matrix were true, the result (3.30) suggests that the limiting distribution, $F_{1}^{(2)}$, of the second largest eigenvalue of GOE is equal to the limiting distribution, $F_{4}$, of the largest eigenvalue of GSE. Equivalently, since a GSE matrix has double eigenvalues, the second eigenvalues of GOE and GSE are expected to have the same limiting distribution. An indication for this is Theorem 10.6.1 of [36], which says that the distributions of $N$ alternate angles of the eigenvalues of a random $2 N \times 2 N$ matrix taken from the circular orthogonal ensemble (COE) are identical to those of the $N$ angles of the eigenvalues of a random $N \times N$ matrix taken from the circular symplectic ensemble (CSE). Indeed for $2 N \times 2 N$ Laguerre ensembles, we have proved that the joint distributions of the second, fourth, sixth, ... largest eigenvalues of the Laguerre orthogonal ensemble (LOE) and the Laguerre symplectic ensemble (LSE) are identical (see Remark 1 to Corollary 7.6 of [7]). In particular, since the $k^{\text {th }}$ largest eigenvalue of a Laguerre ensemble has the same limiting distribution as the corresponding quantity for a Gaussian ensemble, the above remark implies that

$$
F_{4}^{((2 k-1)}=F_{4}^{(2 k)}=F_{1}^{(2 k)}, \quad k=1,2, \cdots
$$

Thus (3.20) and (3.30) imply that the first and the second row of a random involution has the same limiting distribution as the first and the second eigenvalue of GOE, respectively.

Recently in [24], the authors proved that the same property holds true for GOE and GSE. They also proved that, among many things, the $(2 k)^{t h}$ 'eigenvalue' of a superimposition of two random GOE matrices has the 
same distribution as the $k^{\text {th }}$ eigenvalue of a random GUE matrix. In particular, when $k=1$, this implies that

$$
\left(F_{1}^{2}\right)^{(2)}=F_{2},
$$

and hence (3.21) and (3.31) states that the first and the second rows of a random signed involution have the same limiting distribution as the first and the second 'eigenvalues' of the superimposition of two random GOE matrices, respectively.

\section{Poisson generating functions}

We review the results from [7] which we will need in the proof of the theorems of Section 3. As in [7], throughout the paper, the notation $\circledast$ indicates an arbitrary member of the set $\{\square, \nabla, \nabla\}$.

Definition 6. We define the Poisson generating functions for the distributions introduced above:

$$
\begin{aligned}
Q_{l}^{\square}\left(\lambda_{1}, \lambda_{2}\right) & :=e^{-\lambda_{1}-\lambda_{2}} \sum_{n_{1}, n_{2} \geq 0} \frac{\lambda_{1}^{n_{1}} \lambda_{2}^{n_{2}}}{n_{1} ! n_{2} !} \operatorname{Pr}\left(L_{n_{2}, n_{1}}^{\square} \leq l\right), \\
Q_{l}^{\triangleright}\left(\lambda_{1}, \lambda_{2}\right) & :=e^{-\lambda_{1}-\lambda_{2}} \sum_{n_{1}, n_{2} \geq 0} \frac{\lambda_{1}^{n_{1}} \lambda_{2}^{n_{2}}}{n_{1} ! n_{2} !} \operatorname{Pr}\left(L_{n_{2}, n_{1}}^{\square} \leq l\right), \\
Q_{l}^{\bigotimes}\left(\lambda_{1}, \lambda_{2}, \lambda_{3}\right) & :=e^{-\lambda_{1}-\lambda_{2}-\lambda_{3}} \sum_{n_{1}, n_{2}, n_{3} \geq 0} \frac{\lambda_{1}^{n_{1}} \lambda_{2}^{n_{2}} \lambda_{3}^{n_{3}}}{n_{1} ! n_{2} ! n_{3} !} \operatorname{Pr}\left(L_{n_{3}, n_{1}, n_{2}}^{\bowtie} \leq l\right) .
\end{aligned}
$$

As in [7], let $\tilde{f}_{n m l}^{\square}$ (resp., $\tilde{f}_{n m l}^{\square}$ ) be the number of involutions on $n$ numbers with $m$ fixed points with no increasing (resp., decreasing) subsequence of length greater than $l$. Thus $\tilde{f}_{\left(2 n_{2}+n_{1}\right) n_{1} l}^{\square}=\operatorname{Pr}\left(L_{n_{2}, n_{1}}^{\square} \leq l\right) \cdot\left|S_{n_{2}, n_{1}}\right|$, etc. Also let $\tilde{f}_{n m_{+} m_{-} l}^{\square}$ be the number of signed involutions on $2 n$ letters with $2 m_{+}$fixed points and $2 m_{-}$negated points with no increasing subsequence of length greater than $l: \tilde{f}_{\left(2 n_{3}+n_{1}+n_{2}\right) n_{1} n_{2} l}^{\bigotimes}=\operatorname{Pr}\left(L_{n_{3}, n_{1}, n_{2}}^{\otimes} \leq l\right) \cdot\left|S_{n_{3}, n_{1}, n_{2}}^{\otimes}\right|$. We also define

$$
\begin{aligned}
P_{l}^{\square}(t ; \alpha) & :=e^{-\alpha t-t^{2} / 2} \sum_{0 \leq n} \frac{t^{n}}{n !} \sum_{0 \leq m} \alpha^{m} \tilde{f}_{n m l}^{\square}, \\
P_{l}^{\square}(t ; \beta) & :=e^{-\beta t-t^{2} / 2} \sum_{0 \leq n} \frac{t^{n}}{n !} \sum_{0 \leq m} \beta^{m} \tilde{f}_{n m l}^{\square}, \\
P_{l}^{\bigotimes}(t ; \alpha, \beta) & :=e^{-\alpha t-\beta t-t^{2}} \sum_{0 \leq n} \frac{t^{n}}{n !} \sum_{0 \leq m_{+}, m_{-}} \alpha^{m_{+}} \beta^{m_{-}} \tilde{f}_{n m_{+} m_{-} l}^{\bigotimes} .
\end{aligned}
$$

Using $\left|S_{n, m}\right|=\frac{(2 n+m) !}{n ! m ! 2^{n}}$ and $\left|S_{n, m_{+}, m_{-}}^{\bowtie}\right|=\frac{\left(2 n+m_{+}+m_{-}\right) !}{n ! m_{+} ! m_{-} !}$, it is easy to check that

$$
\begin{aligned}
P_{l}^{\square}(t ; \alpha) & =Q_{l}^{\square}\left(\alpha t, t^{2} / 2\right), \\
P_{l}^{\square}(t ; \beta) & =Q_{l}^{\triangleright}\left(\beta t, t^{2} / 2\right), \\
P_{l}^{\bowtie}(t ; \alpha, \beta) & =Q_{l}^{\bigotimes}\left(\alpha t, \beta t, t^{2}\right) .
\end{aligned}
$$

It turns out that the $P$-formulae in (4.4)-(4.6) are useful for algebraic manipulations (see [7]), while the $Q$ formulae (4.1)-(4.3) are well adapted to asymptotic analysis. 
The following results from [7] provide the starting point for our analysis in this paper. For a nonnegative integer $k$, define $\pi_{k}(z ; t)=z^{k}+\cdots$ to be the monic orthogonal polynomial of degree $k$ with respect to the weight function $\exp (t(z+1 / z)) d z /(2 \pi i)$ on the unit circle. Let the norm of $\pi_{k}(z ; t)$ be $N_{k}(t)$ :

$$
\int_{\Sigma} \pi_{n}(z ; t) \overline{\pi_{m}(z ; t)} e^{t(z+1 / z)} \frac{d z}{2 \pi i z}=N_{n}(t) \delta_{n m} .
$$

We note that all the coefficients of $\pi_{n}(z ; t)$ are real. Define

$$
\pi_{n}^{*}(z ; t):=z^{n} \pi_{n}\left(z^{-1} ; t\right)
$$

Then

Theorem 4.1 (Collorary 4.3 and Collorary 2.7 of $[7]$ ). For $\alpha, \beta \geq 0$,

$$
\begin{aligned}
P_{2 l}^{\square}(t ; \alpha) & =e^{-\alpha t-t^{2} / 2} \frac{1}{2}\left\{\left[\pi_{2 l-1}^{*}(-\alpha ; t)-\alpha \pi_{2 l-1}(-\alpha ; t)\right] D_{l}^{--}(t)+\left[\pi_{2 l-1}^{*}(-\alpha ; t)+\alpha \pi_{2 l-1}(-\alpha ; t)\right] D_{l-1}^{++}(t)\right\} \\
P_{2 l+1}^{\square}(t ; \alpha) & =e^{-\alpha t-t^{2} / 2} \frac{1}{2}\left\{\left[\pi_{2 l}^{*}(-\alpha ; t)+\alpha \pi_{2 l}(-\alpha ; t)\right] e^{t} D_{l}^{+-}(t)+\left[\pi_{2 l}^{*}(-\alpha ; t)-\alpha \pi_{2 l}(-\alpha ; t)\right] e^{-t} D_{l}^{-+}(t)\right\} \\
P_{2 l+1}^{\square}(t ; \beta) & =e^{-t^{2} / 2} D_{l}^{++}(t), \\
P_{2 l+1}^{凶}(t ; \alpha, \beta) & =e^{-\alpha t-t^{2}} \pi_{l}^{*}(-\alpha ; t) D_{l}(t),
\end{aligned}
$$

where for any real $t \geq 0, D_{l}(t)$ and $D_{l}^{ \pm \pm}(t)$ are certain Toeplitz and Hankel determinants which in turn can be written as

$$
\begin{aligned}
e^{-t^{2}} D_{l}(t) & =\prod_{j \geq l} N_{j}(t)^{-1}, \\
e^{-t^{2} / 2} D_{l}^{--}(t) & =\prod_{j \geq l} N_{2 j+2}(t)^{-1}\left(1+\pi_{2 j+2}(0 ; t)\right), \\
e^{-t^{2} / 2} D_{l}^{++}(t) & =\prod_{j \geq l} N_{2 j+2}(t)^{-1}\left(1-\pi_{2 j+2}(0 ; t)\right), \\
e^{-t^{2} / 2+t} D_{l}^{+-}(t) & =\prod_{j \geq l} N_{2 j+1}(t)^{-1}\left(1-\pi_{2 j+1}(0 ; t)\right), \\
e^{-t^{2} / 2-t} D_{l}^{-+}(t) & =\prod_{j \geq l} N_{2 j+1}(t)^{-1}\left(1+\pi_{2 j+1}(0 ; t)\right) .
\end{aligned}
$$

Remark. The absence of $\beta$ on the right hand side of (4.14) is fairly simple to explain. Observe that in the point selection model, the longest decreasing subsequence can always be chosen to be symmetric about the diagonal; moreover, any decreasing subsequence can contain at most one diagonal point. Thus if the longest decreasing subsequence has $l$ points, then removing the diagonal points will result in a longest decreasing subsequence with $2[l / 2]$ points. The independence from $\beta$ is thus special to $P_{l}^{\square}$ for $l$ odd; for $l$ even, we do indeed have $\beta$-dependence. But by the monotonicity of $P_{l}^{\triangleright}$ in $l$, we only need (4.14) to compute the limiting distribution; in particular, the limiting distribution will not depend on $\beta$. A similar remark applies to 4.15). 
As a special case:

Theorem 4.2 (Theorem 2.5 and Collorary 4.3 of []]). For $l \geq 0$, we have the following formulae:

$$
\begin{aligned}
P_{2 l+2}^{\square}(t ; 0) & =e^{-t^{2} / 2}\left[D_{l+1}^{--}(t)+D_{l}^{++}(t)\right] / 2, \\
P_{2 l+1}^{\square}(t ; 0) & =e^{-t^{2} / 2}\left[e^{t} D_{l}^{+-}(t)+e^{-t} D_{l}^{-+}(t)\right] / 2, \\
P_{2 l}^{\square}(t ; 0) & =e^{-t^{2} / 2} D_{l}^{++}(t), \\
P_{2 l}^{\square}(t ; 1) & =P_{2 l}^{\square}(t ; 1)=e^{-t-t^{2} / 2} D_{l}^{-+}(t), \\
P_{2 l+1}^{\square}(t ; 1) & =P_{2 l+1}^{\square}(t ; 1)=e^{-t^{2} / 2} D_{l}^{++}(t), \\
P_{2 l}^{\bigotimes}(t ; 0,0) & =e^{-t^{2}} D_{l}(t), \\
P_{4 l+1}^{\bigotimes}(t ; 1, \beta) & =e^{-t-t^{2}} D_{l}^{++}(t) D_{l}^{-+}(t), \\
P_{4 l+3}^{\otimes}(t ; 1, \beta) & =e^{-t-t^{2}} D_{l}^{++}(t) D_{l+1}^{-+}(t) .
\end{aligned}
$$

Also $P_{0}^{\square}(t ; 0)=e^{-t^{2} / 2} D_{0}^{--}(t)=e^{-t^{2} / 2}$.

For the second row, we define the Poisson generating functions in a similar manner. Then we have

Theorem 4.3 (Theorem 5.8 and Collorary 5.12 of $[7]$ ). For $\alpha, \beta \geq 0$,

$$
\begin{aligned}
P_{l}^{\square,(2)}(t ; \alpha) & =P_{l}^{\square}(t ; 0), \\
P_{2 l+1}^{\square,(2)}(t ; \beta) & =P_{2 l}^{\square}(t ; 0), \\
P_{2 l+1}^{\bigotimes,(2)}(t ; \alpha, \beta) & =P_{2 l}^{\bigotimes}(t ; 0,0) .
\end{aligned}
$$

\section{Asymptotics of orthogonal polynomials}

Let $\Sigma=\{z \in \mathbb{C}:|z|=1\}$ be the unit circle in the complex plane, oriented counterclockwise. Set

$$
\psi(z ; t):=e^{t\left(z+z^{-1}\right)} .
$$

Let $\pi_{n}(z ; t)=z^{n}+\cdots$ be the $n$-th monic orthogonal polynomial with respect to the measure $\psi(z ; t) d z /(2 \pi i z)$ on the unit circle. From Theorems 4.1 and 4.2, in order to obtain the asymptotics of the Poisson generating functions, we need the asymptotics, as $k, t \rightarrow \infty$, of

$$
N_{k}(t), \quad \pi_{k}(z ; t), \quad \pi_{k}^{*}(z ; t)
$$

In this section, we summarize the asymptotic results for these quantities.

Define the $2 \times 2$ matrix-valued function of $z$ in $\mathbb{C} \backslash \Sigma$ by

$$
Y(z ; k ; t):=\left(\begin{array}{cc}
\pi_{k}(z ; t) & \int_{\Sigma} \frac{\pi_{k}(s ; t)}{s-z} \frac{\psi(s ; t) d s}{2 \pi i s^{k}} \\
-N_{k-1}(t)^{-1} \pi_{k-1}^{*}(z ; t) & -N_{k-1}(t)^{-1} \int_{\Sigma} \frac{\pi_{k-1}^{*}(s ; t)}{s-z} \frac{\psi(s ; t) d s}{2 \pi i s^{k}}
\end{array}\right), \quad k \geq 1 .
$$


Then $Y(\cdot ; k ; t)$ solves the following Riemann-Hilbert problem (RHP) (see Lemma 4.1 in [3]):

$$
\left\{\begin{array}{l}
Y(z ; k ; t) \quad \text { is analytic in } z \in \mathbb{C} \backslash \Sigma, \\
Y_{+}(z ; k ; t)=Y_{-}(z ; k ; t)\left(\begin{array}{cc}
1 & \frac{1}{z^{k}} \psi(z ; t) \\
0 & 1
\end{array}\right), \quad \text { on } z \in \Sigma, \\
Y(z ; k ; t)\left(\begin{array}{cc}
z^{-k} & 0 \\
0 & z^{k}
\end{array}\right)=I+O\left(\frac{1}{z}\right) \text { as } z \rightarrow \infty .
\end{array}\right.
$$

Here the notation $Y_{+}(z ; k)$ (resp., $Y_{-}$) denotes the limiting value $\lim _{z^{\prime} \rightarrow z} Y\left(z^{\prime} ; k\right)$ with $\left|z^{\prime}\right|<1$ (resp., $\left|z^{\prime}\right|>1$ ). Note that $k$ and $t$ play the role of external parameters in the above RHP ; in particular, the term $O\left(\frac{1}{z}\right)$ does not imply a uniform bound in $k$ and $t$. One can easily show that the solution of the above RHP is unique, hence (5.3) is the unique solution of the above RHP. This RHP formulation of orthogonal polynomials on the unit circle given in [3] is an adaptation of a result of Fokas, Its and Kitaev in [21] where they considered orthogonal polynomials on the real line.

From (5.3), the quantities in (5.2) are equal to

$$
\begin{aligned}
N_{k-1}(t)^{-1} & =-Y_{21}(0 ; k ; t), \\
\pi_{k}(z ; t) & =Y_{11}(z ; k ; t), \\
\pi_{k}^{*}(z ; t) & =z^{k} Y_{11}\left(z^{-1} ; k ; t\right)=Y_{21}(z ; k+1 ; t)\left(Y_{21}(0 ; k+1 ; t)\right)^{-1} .
\end{aligned}
$$

(For the other entries of $Y$, one can check directly from (5.3) that $Y_{12}(0 ; k ; t)=N_{k}(t), Y_{22}(0 ; k ; t)=\pi_{k}(0 ; t)$.) Thus the asymptotic analysis of the RHP (5.4) would yield the asymptotics of the above quantities, and hence eventually the theorems in Section 3. The asymptotic analysis of the RHP (5.4) was conducted in [3] with special interest on $Y_{21}(0 ; k ; t)$. But as mentioned in the Introduction, [3] controlled the solution $Y(z)$ to the RHP (5.4) in a uniform way. In [3] and the theorem below, it is natural to distinguish five different regimes of $k$ and $t$. From the analysis of [3], the following results for $Y(0 ; k ; t)$, except for $\pi_{k}(0 ; t)$ in the case (ii) below, can be directly read off. For example, (5.34)-(5.35) of [3] yield the estimates for the case (iii) below when $x \geq 0$. For the case (ii), we need to improve the $L^{1}$ norm bound, (5.23) in [3], of the associated jump matrix. If one is interested only on $N_{k-1}(t)$, the first integral involving $w^{(3)}(s)$ in the displayed equation before (5.19) of 3 vanishes, and hence the bound (5.23) of [3] was enough. But for $\pi_{k}(0 ; t)$, this integral does not vanish, and we need an improved bound. See the discussion in (10.43)-(10.45) below.

Proposition 5.1 ([3]). There exists $M_{0}>0$ such that as $k, t \rightarrow \infty$, we have the following asymptotic results for $N_{k-1}(t)$ and $\pi_{k}(0 ; t)$ in each different region of $k$ and $t$.

(i). If $0 \leq 2 t \leq a k$ with $0<a<1$, then

$$
\left|N_{k-1}(t)^{-1}-1\right|, \quad\left|\pi_{k}(0 ; t)\right| \leq C e^{-c k},
$$

for some constants $C, c>0$.

(ii). If $a k \leq 2 t \leq k-M k^{1 / 3}$ with some $M>M_{0}$ and $0<a<1$, then

$$
\left|N_{k-1}(t)^{-1}-1\right|, \quad\left|\pi_{k}(0 ; t)\right| \leq \frac{C}{k^{1 / 3}} e^{-\frac{2 \sqrt{2}}{3} k\left(1-\frac{2 t}{k}\right)^{3 / 2}},
$$


for some constant $C>0$.

(iii). If $2 t=k-\frac{x}{2^{1 / 3}} k^{1 / 3}$ with $-M \leq x \leq M$ for some constant $M>0$, then

$$
\left|N_{k-1}(t)^{-1}-1-\frac{2^{1 / 3}}{k^{1 / 3}} v(x)\right|, \quad\left|\pi_{k}(0 ; t)+(-1)^{k} \frac{2^{1 / 3}}{k^{1 / 3}} u(x)\right| \leq \frac{C}{k^{2 / 3}},
$$

for some constant $C>0$, where $u(x)$ and $v(x)$ are defined in (2.1) and (2.5) respectively.

(iv). If $k+M k^{1 / 3} \leq 2 t \leq a k$ with some $M>M_{0}$ and $a>1$, then

$$
\left|\sqrt{\frac{2 t}{k}} e^{k(2 t / k-\log (2 t / k)-1)} N_{k-1}(t)^{-1}-1\right|, \quad\left|(-1)^{k} \sqrt{\frac{2 t}{2 t-k}} \pi_{k}(0 ; t)-1\right| \leq \frac{C}{2 t-k},
$$

for some constant $C>0$.

(v). If $a k \leq 2 t \leq b k$ with $1<a<b$, then

$$
\left|\sqrt{\frac{2 t}{k}} e^{k(2 t / k-\log (2 t / k)-1)} N_{k-1}(t)^{-1}-1\right|, \quad\left|(-1)^{k} \sqrt{\frac{2 t}{2 t-k}} \pi_{k}(0 ; t)-1\right| \leq \frac{C}{k},
$$

for some constant $C>0$.

Now we are interested in $\pi_{k}(z ; t)$. If $z$ is apart from -1 and is fixed, then similar estimates for $Y(z ; k ; t)$ can be obtained from the analysis of [3]. The result below when $x \geq 0$ is (almost) direct from the work of [3]. For the case when $x<0$, the analysis of [3] expresses the bound in terms of the so-called $g$-function, and we need further analysis for this $g$-function. When $z=0$, this $g$-function becomes very simple: $g(0)=\pi i$. See (10.134)-(10.139) below.

Proposition 5.2. For $2 t=k-x(k / 2)^{1 / 3}$, $x$ fixed, and for each fixed $z \in \mathbb{C} \backslash \Sigma$, we have

$$
\begin{aligned}
\lim _{k \rightarrow \infty} e^{t z} \pi_{k}(z ; t) & =0, \quad \lim _{k \rightarrow \infty} e^{t z} \pi_{k}^{*}(z ; t)=1, & & |z|<1, \\
\lim _{k \rightarrow \infty} z^{-k} e^{t z^{-1}} \pi_{k}(z ; t) & =1, \quad \lim _{k \rightarrow \infty} z^{-k} e^{t z^{-1}} \pi_{k}^{*}(z ; t)=0, & & |z|>1 .
\end{aligned}
$$

Corollary 5.3. For $2 t=k-x(k / 2)^{1 / 3}$, x fixed, we have for fixed $\alpha>1$,

$$
\lim _{k \rightarrow \infty} e^{-\alpha t} \pi_{k}(-\alpha ; t)=0, \quad \lim _{k \rightarrow \infty} e^{-\alpha t} \pi_{k}^{*}(-\alpha ; t)=0 .
$$

Proof. Write

$$
\begin{aligned}
e^{-\alpha t} \pi_{k}(-\alpha ; t) & =\alpha^{k} e^{t\left(-\alpha+\alpha^{-1}\right)} \alpha^{-k} e^{-t \alpha^{-1}} \pi_{k}(-\alpha ; t) \\
& =e^{k f(\alpha ; 2 t / k)} \alpha^{-k} e^{-t \alpha^{-1}} \pi_{k}(-\alpha ; t),
\end{aligned}
$$

where

$$
f(\alpha ; \gamma)=\frac{\gamma}{2}\left(-\alpha+\alpha^{-1}\right)+\log \alpha
$$


The function $f(\alpha ; 1)$ is strictly decreasing for $\alpha>0$, and $f(1 ; 1)=0$. Hence $f(\alpha ; 1)<0$ for $\alpha>1$. Note that

$$
f(\alpha ; \gamma)=f(\alpha ; 1)+\frac{\gamma-1}{2}\left(-\alpha+\alpha^{-1}\right)
$$

When $x \leq 0,2 t / k \geq 1$, hence $f(\alpha ; 2 t / k) \leq f(\alpha ; 1)$. On the other hand, when $x>0$, since $2 t / k-1=$ $-x /\left(2^{1 / 3} k^{2 / 3}\right)$, we have $f(\alpha ; 2 t / k) \leq \frac{1}{2} f(\alpha ; 1)$ if $k>\left(\frac{2^{2 / 3} x\left(-\alpha+\alpha^{-1}\right)}{f(\alpha ; 1)}\right)^{3 / 2}$. Therefore (5.14) implies that

$$
\left|e^{-\alpha t} \pi_{k}(-\alpha ; t)\right| \leq e^{\frac{k}{2} f(\alpha ; 1)}\left|(-\alpha)^{-k} e^{-t \alpha^{-1}} \pi_{k}(-\alpha ; t)\right| \rightarrow 0,
$$

as $k \rightarrow \infty$. Similar calculations give the desired result for $\pi_{k}^{*}$.

When $z \rightarrow-1$, (which is required for the proof of Theorem 3.2), the estimates for $Y(z ; k ; t)$ can not be directly read off from the result of [3]. However, with more detailed estimates, the same procedure as in [3] gives us the following restuls. See Subsubsection 10.1.3 (10.73) - 10.83) for the case $x \geq 0$, and Subsubsection $10.2 .3(10.140)-(10.150)$ for the case $x<0$. Recall from Section 2 that $m(\cdot, x)$ solves the RHP for the PII equation (2.15).

Proposition 5.4. Let $2 t=k-x(k / 2)^{1 / 3}$ where $x$ is a fixed number. Set

$$
\alpha=1-\frac{2^{4 / 3} w}{k^{1 / 3}}
$$

We have for $w>0$ fixed,

$$
\begin{aligned}
\lim _{k \rightarrow \infty}(-1)^{k} e^{-t \alpha} \pi_{k}(-\alpha ; t) & =-m_{12}(-i w ; x), \\
\lim _{k \rightarrow \infty} e^{-t \alpha} \pi_{k}^{*}(-\alpha ; t) & =m_{22}(-i w ; x),
\end{aligned}
$$

and for $w<0$ fixed,

$$
\begin{aligned}
\lim _{k \rightarrow \infty}(-\alpha)^{-k} e^{-t \alpha^{-1}} \pi_{k}(-\alpha ; t) & =m_{11}(-i w ; x), \\
\lim _{k \rightarrow \infty} \alpha^{-k} e^{-t \alpha^{-1}} \pi_{k}^{*}(-\alpha ; t) & =-m_{21}(-i w ; x) .
\end{aligned}
$$

Corollary 5.5. For $w<0$, under the same condition as the above proposition, we have

$$
\begin{aligned}
\lim _{k \rightarrow \infty}(-1)^{-k} e^{-t \alpha} \pi_{k}(-\alpha ; t) & =m_{11}(-i w ; x) e^{(8 / 3) w^{3}-2 x w} \\
\lim _{k \rightarrow \infty} e^{-t \alpha} \pi_{k}^{*}(-\alpha ; t) & =-m_{21}(-i w ; x) e^{(8 / 3) w^{3}-2 x w} .
\end{aligned}
$$

Proof. Note that under the stated conditions, we have

$$
e^{t\left(\alpha^{-1}-\alpha\right)} \alpha^{k}=e^{(8 / 3) w^{3}-2 x w+O\left(k^{-1 / 3}\right)} .
$$

Remark. As noted in Section 2, it follows from the RHP 2.15) that $\left(m_{12}\right)_{+}(0 ; x)=-\left(m_{11}\right)_{-}(0 ; x)$ and $\left(m_{22}\right)_{+}(0 ; x)=-\left(m_{21}\right)_{-}(0 ; x)$, and hence by the above Corollary, the limits in (5.21), (5.22) are in fact continuous across $w=0$. 
For convergence of moments, we need uniform bound of $\pi_{k}(z ; t)$ for $|x| \geq M$ for a fixed number $M>0$. The results (5.29) and (5.30) are essentially in the analysis of [3], while (5.31)-(5.34) are new estimates. We again need to extend the method of [3] to obtain the results below. The proof is provided in Section 10. See Subsubsection 10.1.1 and 10.1.2 for the case $x \geq M$, and Subsubsection 10.2.1 and 10.2.2 for the case $x \leq-M$.

Proposition 5.6. Define $x$ through the relation

$$
\frac{2 t}{k}=1-\frac{x}{2^{1 / 3} k^{2 / 3}}
$$

Then there exists $M_{0}$ such that the following holds for any fixed $M>M_{0}$. Let $0<b<1$ and $0<L<2^{-3 / 2} \sqrt{M}$ be fixed. Then as $k, t \rightarrow \infty$, we have for $x \geq M$,

$$
\begin{aligned}
\left|e^{t z} \pi_{k}(z ; t)\right| \leq C e^{-c|x|^{3 / 2}}, & |z| \leq b, \\
\left|e^{t z^{-1}} z^{-k} \pi_{k}(z ; t)-1\right| \leq C e^{-c|x|^{3 / 2}}, & |z| \geq b^{-1}, \\
\left|e^{-t \alpha} \pi_{k}(-\alpha ; t)\right| \leq C e^{c|x|}, & \alpha=1-2^{4 / 3} k^{-1 / 3} w, \quad-L \leq w \leq L, \\
\left|e^{-t \alpha^{-1}}(-\alpha)^{-k} \pi_{k}(-\alpha ; t)-1\right| \leq C e^{-c|x|^{3 / 2}}, & \alpha=1-2^{4 / 3} k^{-1 / 3} w, \quad-L \leq w \leq L,
\end{aligned}
$$

and for $x \leq-M$,

$$
\begin{aligned}
\left|e^{-t \alpha} \pi_{k}(-\alpha ; t)\right| & \leq C, & & 0<\alpha \leq 1, \\
\left|e^{-t \alpha^{-1}}(-\alpha)^{-k} \pi_{k}(-\alpha ; t)\right| & \leq C, & & \alpha \geq 1 .
\end{aligned}
$$

Corollary 5.7. Let $\alpha=1-2^{4 / 3} w k^{-1 / 3}$ and $-L \leq w \leq L$ for fixed $L>0$. Under the assumption of the above proposition, for $x \leq-M$, we have

$$
\begin{aligned}
\left|e^{-t \alpha} \pi_{k}(-\alpha ; t)\right| & \leq C e^{c|x|}, \\
\left|e^{-t \alpha^{-1}}(-\alpha)^{-k} \pi_{k}(-\alpha ; t)\right| & \leq C e^{c|x|} .
\end{aligned}
$$

for some positive constants $C, c$.

Proof. We have

$$
\begin{aligned}
\left|e^{-t\left(\alpha-\alpha^{-1}\right)} \alpha^{k}\right| & =e^{2 x w+\frac{8}{3} w^{3}+O\left(k^{-1}\right)}, \\
\left|e^{t\left(\alpha-\alpha^{-1}\right)} \alpha^{-k}\right| & =e^{-2 x w-\frac{8}{3} w^{3}+O\left(k^{-1}\right)} .
\end{aligned}
$$

Above proposition shows that (5.35) is true for $w \geq 0$. For $w<0$, write

$$
e^{-t \alpha} \pi_{k}(-\alpha ; t)=\left[e^{-t\left(\alpha-\alpha^{-1}\right)}(-\alpha)^{k}\right]\left[e^{-t \alpha^{-1}}(-\alpha)^{-k} \pi_{k}(-\alpha ; t)\right] .
$$

Now (5.35) follows from (5.34) and (5.37). The estimate (5.38) is proved similarly.

The result below is new and is used for the asymptotics of $L_{n,[\sqrt{2 n} \alpha]}^{\square}$ when $\alpha>1$. See Subsection 10.3 for the proof. 
Proposition 5.8. Let $\alpha>1$ be fixed. When

$$
\frac{t}{k}=\frac{\alpha}{\alpha^{2}+1}-\frac{\alpha\left(\alpha^{2}-1\right)^{1 / 2}}{\left(\alpha^{2}+1\right)^{3 / 2}} \cdot \frac{x}{\sqrt{k}}, \quad x \text { fixed },
$$

we have

$$
\lim _{k \rightarrow \infty} e^{-\alpha t}(-\alpha)^{k} \pi_{k}\left(-\alpha^{-1} ; t\right)=\frac{1}{\sqrt{2 \pi}} \int_{-\infty}^{x} e^{-\frac{1}{2} y^{2}} d y
$$

\section{De-Poissonization lemmas}

In this section, we describe a series of Tauberian type de-Poissonization lemmas, which enable us to extract the asymptotics of the coefficient from the knowledge of the asymptotics of its generating function. Lemma 6.1 below is due to Johansson [30] and Lemma 6.2 is taken from Section 8 in [3. Lemma 6.3 and Lemma 6.4 are multi-index versions. Lemma 6.1 and 6.3 are enough for both convergence in distribution and the convergence of moments, but for convenience of computations, we use Lemma 6.2 and 6.4 for the convergence of moments in the subsequent sections.

For a sequence $q=\left\{q_{n}\right\}_{n \geq 0}$, we define its Poisson generating function by

$$
\phi(\lambda)=e^{-\lambda} \sum_{0 \leq n} q_{n} \frac{\lambda^{n}}{n !} .
$$

Lemma 6.1. For any fixed real number $d>0$, set

$$
\begin{aligned}
& \mu_{n}^{(d)}=n+(2 \sqrt{d+1}+1) \sqrt{n \log n}, \\
& \nu_{n}^{(d)}=n-(2 \sqrt{d+1}+1) \sqrt{n \log n} .
\end{aligned}
$$

Then there are constants $C$ and $n_{0}$ such that for any sequence $q=\left\{q_{n}\right\}_{n \geq 0}$ satisfying (i) $q_{n} \geq q_{n+1}$ (ii) $0 \leq q_{n} \leq 1$, for all $n \geq 0$,

$$
\phi\left(\mu_{n}^{(d)}\right)-C n^{-d} \leq q_{n} \leq \phi\left(\nu_{n}^{(d)}\right)+C n^{-d}
$$

for all $n \geq n_{0}$.

Lemma 6.2. For any fixed real number $d>0$, there exist constants $C$ and $n_{0}$ such that for any sequence $q=\left\{q_{n}\right\}_{n \geq 0}$ satisfying (i) and (ii) above,

$$
\begin{aligned}
q_{n} & \leq C \phi(n-d \sqrt{n}), \\
1-q_{n} & \leq C(1-\phi(n+d \sqrt{n}))
\end{aligned}
$$

for all $n \geq n_{0}$.

For multi-indexed sequences, there are similar results. For $q=\left\{q_{n_{1}, n_{2}}\right\}_{n_{1}, n_{2} \geq 0}$, define

$$
\phi\left(\lambda_{1}, \lambda_{2}\right)=e^{-\lambda_{1}-\lambda_{2}} \sum_{n_{1}, n_{2} \geq 0} q_{n_{1} n_{2}} \frac{\lambda_{1}^{n_{1}} \lambda_{2}^{n_{2}}}{n_{1} ! n_{2} !} .
$$

From the above two lemmas, we easily obtain the following lemmas. 
Lemma 6.3. For any real number $d>0$, define $\mu_{n}^{(d)}$ and $\nu_{n}^{(d)}$ as in Lemma 6.1. Then there exist constants $C$ and $n_{0}$ such that for any $q=\left\{q_{n_{1}, n_{2}}\right\}_{n_{1}, n_{2} \geq 0}$ satisfying (i) $q_{n_{1}, n_{2}} \geq q_{n_{1}+1, n_{2}}, q_{n_{1}, n_{2}} \geq q_{n_{1}, n_{2}+1}$ (ii) $0 \leq q_{n_{1}, n_{2}} \leq$ 1 , for all $n_{1}, n_{2} \geq 0$,

$$
\phi\left(\mu_{n_{1}}^{(d)}, \mu_{n_{2}}^{(d)}\right)-C\left(n_{1}^{-d}+n_{2}^{-d}\right) \leq q_{n_{1} n_{2}} \leq \phi\left(\nu_{n_{1}}^{(d)}, \nu_{n_{2}}^{(d)}\right)+C\left(n_{1}^{-d}+n_{2}^{-d}\right)
$$

for all $n_{1}, n_{2} \geq n_{0}$.

Similarly,

Lemma 6.4. For any fixed real number $d>0$, there exist constants $C$ and $n_{0}$ such that for any $q=\left\{q_{n_{1}, n_{2}}\right\}_{n_{1}, n_{2} \geq 0}$ satisfying two condition in Lemma 6.5,

$$
\begin{aligned}
q_{n_{1} n_{2}} & \leq C \phi\left(n_{1}-d \sqrt{n_{1}}, n_{2}-d \sqrt{n_{2}}\right), \\
1-q_{n_{1} n_{2}} & \leq C\left(1-\phi\left(n_{1}-d \sqrt{n_{1}}, n_{2}+d \sqrt{n_{2}}\right)\right)
\end{aligned}
$$

for $n_{1}, n_{2} \geq n_{0}$.

Remark. Similar lemmas hold true for sequences of arbitrarily many indices.

\section{Proofs of Theorems [3.1, [3.2, 3.3 and 3.5}

The following results follow from Proposition 5.1 above. The result (7.1) is derived in Lemma 7.1 (iii) of [3], and the other cases are similar. We omit the details.

Corollary 7.1. Let $M>M_{0}$, where $M_{0}$ is given in Proposition 5.8. Then there exist positive constants $C$ and $c$ which are independent of $M$, and a positive constant $C(M)$ which may depend on $M$, such that the following results hold for large $l$.

(i) 园] Define $x$ by $2 t=l-x(l / 2)^{1 / 3}$. For $-M<x<M$,

$$
\left|\sum_{j \geq l} \log N_{j}(t)^{-1}-2 \log F(x)\right| \leq \frac{C(M)}{l^{1 / 3}}+C e^{-c M^{3 / 2}} .
$$

(ii) Define $x$ by $t=l-(x / 2) l^{1 / 3}$. For $-M<x<M$,

$$
\begin{gathered}
\left|\sum_{j \geq l} \log N_{2 j+2}(t)^{-1}-\log F(x)\right|, \quad\left|\sum_{j \geq l} \log N_{2 j+1}(t)^{-1}-\log F(x)\right| \leq \frac{C(M)}{l^{1 / 3}}+C e^{-c M^{3 / 2}}, \\
\left|\sum_{j \geq l} \log \left(1-\pi_{2 j+2}(0 ; t)\right)-\log E(x)\right|,\left|\sum_{j \geq l} \log \left(1+\pi_{2 j+1}(0 ; t)\right)-\log E(x)\right| \leq \frac{C(M)}{l^{1 / 3}}+C e^{-c M^{3 / 2}}, \\
\left|\sum_{j \geq l} \log \left(1+\pi_{2 j+2}(0 ; t)\right)+\log E(x)\right|,\left|\sum_{j \geq l} \log \left(1-\pi_{2 j+1}(0 ; t)\right)+\log E(x)\right| \leq \frac{C(M)}{l^{1 / 3}}+C e^{-c M^{3 / 2}} .
\end{gathered}
$$

These results yield the asymptotics of the determinants in Theorem 4.1 . 
Corollary 7.2. There exits $M_{1}$ such that for $M>M_{1}$, there exist positive constants $C$ and $c$ which are independent of $M$, and a positive constant $C(M)$ which may depend on $M$ such that the following results hold for large $l$.

(i) Define $x$ by $2 t=l-x(l / 2)^{1 / 3}$. For $-M<x<M$,

$$
\left|e^{-t^{2}} D_{l}(t)-F(x)^{2}\right| \leq \frac{C(M)}{l^{1 / 3}}+C e^{-c M^{3 / 2}} .
$$

(ii) Define $x$ by $t=l-(x / 2) l^{1 / 3}$. For $-M<x<M$,

$$
\begin{aligned}
\left|e^{-t^{2} / 2} D_{l}^{--}(t)-F(x) E(x)^{-1}\right| & \leq \frac{C(M)}{l^{1 / 3}}+C e^{-c M^{3 / 2}}, \\
\left|e^{-t^{2} / 2} D_{l-1}^{++}(t)-F(x) E(x)\right| & \leq \frac{C(M)}{l^{1 / 3}}+C e^{-c M^{3 / 2}}, \\
\left|e^{-t^{2} / 2+t} D_{l}^{+-}(t)-F(x) E(x)^{-1}\right| & \leq \frac{C(M)}{l^{1 / 3}}+C e^{-c M^{3 / 2}}, \\
\left|e^{-t^{2} / 2-t} D_{l}^{-+}(t)-F(x) E(x)\right| & \leq \frac{C(M)}{l^{1 / 3}}+C e^{-c M^{3 / 2}} .
\end{aligned}
$$

Proof. For $C$ and $c$ in the above corollary, take $M_{1}>M_{0}$ such that $C e^{-c M_{1}^{3 / 2}} \leq \frac{1}{2}$. Once we fix $M>M_{1}$, then for $l$ is large, $\frac{C(M)}{l^{1 / 3}}+C e^{-c M^{3 / 2}}<1$, and hence by (7.1) above, $\left|\sum_{j \geq l} \log N_{j}(t)^{-1}-2 \log F(x)\right| \leq 1$. Using $\left|e^{x}-1\right| \leq(e-1)|x|$ for $|x| \leq 1$,

$$
\begin{aligned}
\left|e^{-t^{2}} D_{l}(t)-F(x)^{2}\right| & =F(x)^{2}\left|e^{\left(\sum_{j \geq l} \log N_{j}(t)^{-1}-2 \log F(x)\right)}-1\right| \\
& \leq(e-1) F(x)^{2}\left|\sum_{j \geq l} \log N_{j}(t)^{-1}-2 \log F(x)\right| .
\end{aligned}
$$

But from (2.11) and (2.13), $F(x)$ is bounded for $x \in \mathbb{R}$. Hence using (7.1), we obtain the result for (i) with new constants $C, c$ and $C(M)$. For (ii), we note that $F(x) E(x)$ and $F(x) E(x)^{-1}$ are bounded for $x \in \mathbb{R}$ from (2.11)-(2.14).

From Proposition 5.2, Corollary 5.3 and Theorems 4.1, 4.2, this Corollary immediately yields the following asymptotics for Poisson generating functions.

Proposition 7.3. Let $2 t=l-x(l / 2)^{1 / 3}$ where $x$ is fixed. As $l \rightarrow \infty$, for each fixed $\alpha, \beta$,

$$
\begin{array}{lll}
P_{l}^{\square}(t ; \alpha) & \rightarrow F_{4}(x), & 0 \leq \alpha<1, \\
P_{l}^{\square}(t ; 1) \rightarrow F_{1}(x), & \\
P_{l}^{\square}(t ; \alpha) \rightarrow 0, & \alpha>1, \\
P_{l}^{\square}(t ; \beta) \rightarrow F_{1}(x), & \beta \geq 0 .
\end{array}
$$

Let $4 t=l-x(2 l)^{1 / 3}$ where $x$ is fixed. As $l \rightarrow \infty$, for each fixed $\alpha, \beta$,

$$
\begin{aligned}
& P_{l}^{\bigotimes}(t ; \alpha, \beta) \rightarrow F_{2}(x), \quad 0 \leq \alpha<1, \beta \geq 0, \\
& P_{l}^{\bigotimes}(t ; 1, \beta) \rightarrow F_{1}(x)^{2}, \quad \beta \geq 0, \\
& P_{l}^{\bigotimes}(t ; \alpha, \beta) \rightarrow 0, \quad \alpha>1, \quad \beta \geq 0 .
\end{aligned}
$$


Similarly, using Proposition 5.4 and Corollary 5.5 and Theorem 4.1, we have:

Theorem 7.4. Let $2 t=l-x(l / 2)^{1 / 3}$ where $x$ is fixed. As $l \rightarrow \infty$, we have for any fixed $w \in \mathbb{R}$,

$$
P_{l}^{\square}(t ; \alpha) \rightarrow F^{\square}(x ; w), \quad \alpha=1-\frac{2^{4 / 3} w}{l^{1 / 3}} .
$$

Let $4 t=l-x(2 l)^{1 / 3}$ where $x$ is fixed. As $l \rightarrow \infty$, we have for each fixed $\beta$ and $w \in \mathbb{R}$,

$$
P_{l}^{\otimes}(t ; \alpha, \beta) \rightarrow F^{\otimes}(x ; w), \quad \alpha=1-\frac{2^{5 / 3} w}{l^{1 / 3}}, \beta \geq 0 .
$$

Recall the relation between $Q_{l}^{\circledast}(\lambda)$ and $P_{l}^{\circledast}(t)$ in (4.7)-(4.9). We now use the de-Poissonization Lemma 6.3 to obtain the asymptotic results of Theorem 3.1 and 3.2. In order to apply the de-Poissonization Lemma, we need the following monotonicity results.

Lemma 7.5 (Monotonicity). For any $l, \operatorname{Pr}\left(L_{k, m}^{\square} \leq l\right), \operatorname{Pr}\left(L_{k, m}^{\vee} \leq l\right)$ and $\operatorname{Pr}\left(L_{k, m_{+}, m_{-}}^{\bigotimes} \leq l\right)$ are monotone decreasing in $k, m, m_{+}$and $m_{-}$.

Proof. We first consider $\operatorname{Pr}\left(L_{k, m}^{\square} \leq l\right)$. Let $f_{k m}:=\operatorname{Pr}\left(L_{k, m}^{\square} \leq l\right) \cdot\left|S_{k, m}\right|$ be the number of elements in $S_{k, m}$ with no increasing subsequence greater than $l$. Consider the map $h: S_{k, m-1} \times\{1,2, \cdots, 2 k+m\} \rightarrow S_{k, m}$ defined as follows: for $(\pi, j) \in S_{k, m-1} \times\{1,2, \cdots, 2 k+m\}$, set $h(\pi, j)(x)=\pi(x)$ for $1 \leq x<j-1, h(\pi, j)(j)=j$, and $h(\pi, j)(x)=\pi(x-1)$ for $j<x \leq 2 k+m$. Then it is easy to see that $h^{-1}(\sigma)$ consists of $m$ elements, hence $(2 k+m)\left|S_{k, m-1}\right|=m\left|S_{k, m}\right|$. Moreover if $\pi \in S_{k, m-1}$ has an increasing subsequence of length greater than $l$, then $h(\pi, j)$ has an increasing subsequence of length greater than $l$. Thus $(2 k+m) f_{k(m-1)} \geq m f_{k m}$. But since $\left|S_{k, m}\right|=\frac{(2 k+m) !}{2^{k} k ! m !}$, we obtain $\operatorname{Pr}\left(L_{k, m-1}^{\square} \leq l\right) \geq \operatorname{Pr}\left(L_{k, m}^{\square} \leq l\right)$.

A similar argument works for the other cases. Note that $\left|S_{k, m_{+}, m_{-}}^{\bigotimes}\right|=\frac{\left(2 k+m_{+}, m_{-}\right) !}{k ! m_{+} ! m_{-} !}$.

Thus Lemma (6.3) can be applied to obtain the asymptotics results in Theorems 3.1 and 3.2. The proofs are similar to that in Section 9 of [3].

Now we consider convergence of moments. For this, we first obtain the following estimates which follow from Proposition 5.1 (i),(ii),(iv),(v) above. The proof is very similar to that of Lemma 7.1 (i),(ii),(iv),(v) of [3]. Compare the results with (2.11)-(2.14) noting Corollary 7.1 .

Corollary 7.6. Set

$$
t=l-\frac{x}{2} l^{1 / 3} .
$$

There exits $M_{2}$ such that for a fixed $M>M_{2}$, there are positive constants $C=C(M)$ and $c=c(M)$ such that the following results hold.

(i). For $x \geq M$,

$$
\begin{aligned}
1-\prod_{j \geq l} N_{2 j+2}(t)^{-1}, \quad 1-\prod_{j \geq l} N_{2 j+1}(t)^{-1} & \leq C e^{-c|x|^{3 / 2}}, \\
1-\prod_{j \geq l}\left(1-\pi_{2 j+2}(0 ; t)\right), \quad 1-\prod_{j \geq l}\left(1+\pi_{2 j+1}(0 ; t)\right) & \leq C e^{-c|x|^{3 / 2}}, \\
1-\prod_{j \geq l}\left(1+\pi_{2 j+2}(0 ; t)\right), \quad 1-\prod_{j \geq l}\left(1-\pi_{2 j+1}(0 ; t)\right) & \leq C e^{-c|x|^{3 / 2}},
\end{aligned}
$$


(ii). For $x \leq-M$,

$$
\begin{aligned}
& \prod_{j \geq l} N_{2 j+2}(t)^{-1}, \quad \prod_{j \geq l} N_{2 j+1}(t)^{-1} \leq C e^{-c|x|^{3}}, \\
& \prod_{j \geq l}\left(1-\pi_{2 j+2}(0 ; t)\right), \quad \prod_{j \geq l}\left(1+\pi_{2 j+1}(0 ; t)\right) \leq C e^{-c|x|^{3 / 2}}, \\
& \prod_{j \geq l}\left(1+\pi_{2 j+2}(0 ; t)\right), \quad \prod_{j \geq l}\left(1-\pi_{2 j+1}(0 ; t)\right) \leq C e^{+c|x|^{3 / 2}} .
\end{aligned}
$$

Remark. From the definitions of $P_{l}^{\circledast}(t)$ and the equalities of Theorem 4.1, we know that all the infinite products above are between 0 and 1 .

Now as in Section 9 of [3], using Lemma 6.4 and Theorems 3.1, 3.2, this implies Theorem 3.3.

Theorem 3.5 follows from Theorem 4.3 .

\section{Proofs of Theorems 3.4 and 3.6}

In this section, we prove Theorem 3.4 by summing up the asymptotic results of Theorems 3.1, 3.2 and 3.3 . Theorem 3.6 can be proved in a similar way from Theorem 4.3 .

Proof of (3.20). Note that we have a disjoint union

$$
\tilde{S}_{n}=\bigcup_{2 k+m=n} S_{k, m}
$$

Set $p_{k m l}^{\square}=\operatorname{Pr}\left(L_{k m}^{\square} \leq l\right)$, the probability that the length of the longest decreasing subsequence of $\pi \in S_{k, m}$ is less than or equal to $l$. As the first row and the first column of $\pi$ in $\tilde{S}_{n}$ have the same statistics, we have

$$
\operatorname{Pr}\left(\tilde{L}_{n} \leq l\right)=\frac{1}{\left|\tilde{S}_{n}\right|} \sum_{2 k+m=n} p_{k m l}^{\triangleright}\left|S_{k, m}\right| .
$$

Note that

$$
\left|S_{k, m}\right|=\left(\begin{array}{c}
2 k+m \\
2 k
\end{array}\right) \frac{(2 k) !}{2^{k} k !} .
$$

As $n \rightarrow \infty$ (see pp.66-67 of [33]), we have

$$
\left|\tilde{S}_{n}\right|=\sum_{2 k+m=n}\left|S_{k, m}\right|=\frac{1}{\sqrt{2}} n^{n / 2} e^{-n / 2+\sqrt{n}-1 / 4}\left(1+\frac{7}{24} n^{-1 / 2}+O\left(n^{-3 / 4}\right)\right),
$$

and the main contribution to the sum comes from $\sqrt{n}-n^{\epsilon+1 / 4} \leq m \leq \sqrt{n}+n^{\epsilon+1 / 4}$.

Fix $0<a<1<b$. We split the sum in (8.2) into two pieces :

$$
\operatorname{Pr}\left(\tilde{L}_{n} \leq l\right)=\frac{1}{\left|\tilde{S}_{n}\right|}\left[\sum_{(*)} p_{k m l}^{\triangleright}\left|S_{k, m}\right|+\sum_{(* *)} p_{k m l}^{\triangleright}\left|S_{k, m}\right|\right],
$$


where $(*)$ is the region $a \sqrt{n} \leq m \leq b \sqrt{n}$ and $(* *)$ is the rest.

For $2 k+m=n$, the quantity $\left|S_{k, m}\right|=\left(\begin{array}{c}n \\ 2 k\end{array}\right) \frac{(2 k) !}{2^{k} k !}$ is unimodal for $0 \leq k \leq n$, and the maximum is achieved when $k \sim(n-\sqrt{n}) / 2$ as $n \rightarrow \infty$. Hence

$$
\sum_{(* *)} p_{k m l}^{\triangleright}\left|S_{k, m}\right| \leq n \cdot \max \left(\left|S_{k,[a \sqrt{n}]}\right|, \quad\left|S_{k,[b \sqrt{n}]}\right|\right) .
$$

Using Stirling's formula for (8.3), for any fixed $c$, when $2 k+[c \sqrt{n}]=n$,

$$
\left|S_{k,[c \sqrt{n}]}\right| \sim n^{n / 2} e^{-n / 2+\sqrt{n}(c-c \log c)} \frac{e^{-1 / 2+c^{2} / 4}}{\sqrt{\pi c} n^{1 / 4}} .
$$

Hence using (8.4), we have

$$
\frac{1}{\left|\tilde{S}_{n}\right|} \sum_{(* *)} p_{k m l}^{\triangleright}\left|S_{k, m}\right| \leq C n^{3 / 4} \cdot \max \left(e^{\sqrt{n}(a-1-a \log a)}, e^{\sqrt{n}(b-1-b \log b)}\right) .
$$

But $f(x)=x-1-x \log x$ is increasing in $0<x<1$, is decreasing in $x>1$, and $f(1)=0$. Therefore there are positive constants $C$ and $c$ such that for large $n$,

$$
\frac{1}{\left|\tilde{S}_{n}\right|} \sum_{(* *)} p_{k m l}^{\triangleright}\left|S_{k, m}\right| \leq C e^{-c \sqrt{n}} .
$$

On the other hand, Lemma 6.3 says that (recall (4.8)) for any fixed real number $d>0$, there is a constant $C$ such that for $a \sqrt{n} \leq m \leq b \sqrt{n}$,

$$
\begin{aligned}
P_{l}^{\triangleright}\left(\left(2 \mu_{k}^{(d)}\right)^{1 / 2}\right. & \left.; \mu_{m}^{(d)}\left(2 \mu_{k}^{(d)}\right)^{-1 / 2}\right)-C n^{-d / 2} \\
& \leq p_{k m l}^{\triangleright} \leq P_{l}^{\nabla}\left(\left(2 \nu_{k}^{(d)}\right)^{1 / 2} ; \nu_{m}^{(d)}\left(2 \nu_{k}^{(d)}\right)^{-1 / 2}\right)+C n^{-d / 2},
\end{aligned}
$$

for sufficiently large $n$. Since $P_{l}^{\square}(t ; \beta) \leq P_{l+1}^{\square}(t ; \beta)$, Theorem 4.1 for $P_{2 l+1}^{\square}(t ; \beta)$ yields

$$
e^{-\mu_{k}^{(d)}} D_{[(l-1) / 2]}^{++}\left(\left(2 \mu_{k}^{(d)}\right)^{1 / 2}\right)-C n^{-d / 2} \leq p_{n m l}^{\triangleright} \leq e^{-\nu_{k}^{(d)}} D_{[l / 2]}^{++}\left(\left(2 \nu_{k}^{(d)}\right)^{1 / 2}\right)+C n^{-d / 2} .
$$

Let $l=\left[2 \sqrt{n}+x n^{1 / 6}\right]$. For $a \sqrt{n} \leq m \leq b \sqrt{n}$, hence for $\frac{n-b \sqrt{n}}{2} \leq k \leq \frac{n-a \sqrt{n}}{2}$,

$$
\left(l / 2-\left(2 \mu_{k}^{(d)}\right)^{1 / 2}\right) 2(l / 2)^{-1 / 3}, \quad\left(l / 2-\left(2 \nu_{k}^{(d)}\right)^{1 / 2}\right) 2(l / 2)^{-1 / 3}=x+O\left(n^{-1 / 6} \sqrt{\log n}\right) .
$$

Also note that from the asymptotics (2.3), 2.4) and (2.11)-(2.14),

$$
(F(x) E(x))^{\prime}=-\frac{1}{2}(v(x)+u(x)) F(x) E(x)
$$

is bounded for $x \in \mathbb{R}$. Hence using (7.7) in Corollary 7.2, (8.12) and 8.13), we obtain

$$
\begin{aligned}
& \left|e^{-\nu_{k}^{(d)}} D_{[l / 2]}^{++}\left(\left(2 \nu_{k}^{(d)}\right)^{1 / 2}\right)-(F E)(x)\right| \\
& \leq\left|e^{-\nu_{k}^{(d)}} D_{[l / 2]}^{++}\left(\left(2 \nu_{k}^{(d)}\right)^{1 / 2}\right)-(F E)\left(\left(l / 2-\left(2 \nu_{k}^{(d)}\right)^{1 / 2}\right) 2(l / 2)^{-1 / 3}\right)\right| \\
& \quad+\left|(F E)\left(\left(l / 2-\left(2 \nu_{k}^{(d)}\right)^{1 / 2}\right) 2(l / 2)^{-1 / 3}\right)-(F E)(x)\right| \\
& \leq C(M) n^{-1 / 6}+C e^{-c M^{3 / 2}}+C n^{-1 / 6} \sqrt{\log n} .
\end{aligned}
$$


Therefore we have

$$
\sum_{(*)} p_{n m l}^{\square}\left|S_{n, m}\right| \leq\left(F(x) E(x)+C(M) n^{-1 / 6}+C e^{-c M^{3 / 2}}+C n^{-1 / 6} \sqrt{\log n}\right) \sum_{(*)}\left|S_{n, m}\right| .
$$

Similarly,

$$
\sum_{(*)} p_{n m l}^{\square}\left|S_{n, m}\right| \geq\left(F(x) E(x)-C(M) n^{-1 / 6}-C e^{-c M^{3 / 2}}-C n^{-1 / 6} \sqrt{\log n}\right) \sum_{(*)}\left|S_{n, m}\right| .
$$

But from 8.9 ,

$$
\frac{1}{\left|\tilde{S}_{n}\right|} \sum_{(*)}\left|S_{n, m}\right|=1-\frac{1}{\left|\tilde{S}_{n}\right|} \sum_{(* *)}\left|S_{n, m}\right|=1+O\left(e^{-c \sqrt{n}}\right) .
$$

Thus using (8.5), (8.9), 8.15), (8.16) and (8.17), we obtain (3.20).

Proof of (3.22). As in Section 9 in [3], integrating by parts,

$$
\mathrm{E}\left(\left(\tilde{\chi}_{n}\right)^{p}\right)=\int_{-\infty}^{\infty} x^{p} d F_{n}(x)=-\int_{-\infty}^{0} p x^{p-1} F_{n}(x) d x+\int_{0}^{\infty} p x^{p-1}\left(1-F_{n}(x)\right) d x,
$$

where $F_{n}(x):=\operatorname{Pr}\left(\tilde{\chi}_{n} \leq x\right)=\operatorname{Pr}\left(\tilde{L}_{n} \leq 2 \sqrt{n}+x n^{1 / 6}\right)$. From Theorem 4.1 and Corollary 7.6, we have

$$
\begin{aligned}
1-e^{-t^{2} / 2} D_{l}^{++}(t) & \leq C e^{-c|x|^{3 / 2}}, \quad x \geq M, \\
e^{-t^{2} / 2} D_{l}^{++}(t) & \leq C e^{-c|x|^{3}}, \quad x \leq-M,
\end{aligned}
$$

for a fixed $M>M_{2}$ where $t=l-(x / 2) l^{1 / 3}$. Noting that $P_{2 l+1}^{\bowtie}(t ; \beta)=e^{-t^{2} / 2} D_{l}^{++}(t)$ for all $\beta \geq 0$, from (8.2), Lemma 6.4 and (8.19), 8.20), we obtain

$$
\begin{aligned}
1-F_{n}(x) & \leq C e^{-c|x|^{3 / 2}}, \quad x \geq M, \\
F_{n}(x) & \leq C e^{-c|x|^{3}}, \quad x \leq-M .
\end{aligned}
$$

Now using convergence in distribution, the dominated convergence theorem gives (3.22).

Remark. We could also proceed using

$$
\operatorname{Pr}\left(\tilde{L}_{n} \leq l\right)=\frac{1}{\left|\tilde{S}_{n}\right|} \sum_{2 k+m=n} p_{k m l}^{\square}\left|S_{k, m}\right| .
$$

The main contribution to the sum from $\left|S_{k, m}\right|$ comes from the region $|m-\sqrt{n}| \leq n^{1 / 4+\epsilon}$. On the other hand, from Theorem 3.2, when $m=\sqrt{n}-2 w n^{1 / 3}$, the quantity $p_{k m l}^{\square}$ converges to $F(x ; w)$. Since the region $m=\sqrt{n}+c n^{1 / 4+\epsilon}$ is much narrower than the region $m=\sqrt{n}+c n^{1 / 3}$, the main contribution to the sum comes from when $w=0$, implying that

$$
\operatorname{Pr}\left(\tilde{L}_{n} \leq l\right) \sim \frac{1}{\left|\tilde{S}_{n}\right|} \sum_{m=0}^{n} F(x ; 0)\left|S_{n, m}\right|=F_{1}(x) .
$$

In the following proof for signed involutions, we make this argument rigorous. 
Proof of (3.21). We have a disjoint union

$$
\tilde{S}_{n}^{\bigotimes}=\bigcup_{2 k+m_{+}+m_{-}=n} S_{k, m_{+}, m_{-}}^{\bigotimes}
$$

Hence again

$$
\operatorname{Pr}\left(\tilde{L}_{n}^{\bigotimes} \leq l\right)=\frac{1}{\left|\tilde{S}_{n}^{\otimes}\right|} \sum_{2 k+m_{+}+m_{-}=n} p_{k m_{+} m_{-} l}^{\otimes}\left|S_{k, m_{+}, m_{-}}^{\bigotimes}\right| .
$$

One can check that

$$
\left|S_{k, m_{+}, m_{-}}^{\bigotimes}\right|=\frac{\left(2 k+m_{+}+m_{-}\right) !}{k ! m_{+} ! m_{-} !} .
$$

Hence, we have

$$
\left|\tilde{S}_{n}^{\bowtie}\right|=\sum_{2 k+m_{+}+m_{-}=n}\left|S_{k, m_{+}, m_{-}}^{\bigotimes}\right|=\sum_{0 \leq k \leq\left[\frac{n}{2}\right]} \sum_{0 \leq m_{+} \leq n-2 k} f\left(m_{+}, k\right)
$$

where

$$
f\left(m_{+}, k\right):=\frac{n !}{m_{+} !\left(n-m_{+}-2 k\right) ! k !}
$$

For fixed $0 \leq k \leq\left[\frac{n}{2}\right], f\left(m_{+}, k\right)$ is unimodal in $m_{+}$and achieves its maximum when $m_{+} \sim n / 2-k$. And $f(n / 2-k, k)$ is unimodal in $k$ and the maximum is attained when $k \sim n / 2-\sqrt{n / 2}$. Hence $f\left(m_{+}, k\right)$ has its maximum when $\left(m_{+}, k\right) \sim\left(\sqrt{\frac{n}{2}}, \frac{n}{2}-\sqrt{\frac{n}{2}}\right)$. Consider the disc $D$ of radius $n^{1 / 4+\epsilon}$ centered at $\left(\sqrt{\frac{n}{2}}, \frac{n}{2}-\sqrt{\frac{n}{2}}\right)$. We will show that the main contribution to the sum in (8.28) comes from $D$. Set

$$
m_{+}=\sqrt{\frac{n}{2}}+x, \quad k=\frac{n}{2}-\sqrt{\frac{n}{2}}+y, \quad|x|,|y| \leq n^{1 / 4+\epsilon},
$$

By Stirling's formula,

$$
f\left(m_{+}, k\right)=\frac{1}{\sqrt{e n} \pi}(2 n)^{n / 2} e^{-n / 2+\sqrt{2 n}} e^{-\frac{x^{2}+(x+2 y)^{2}}{\sqrt{2 n}}}\left(1+O\left(n^{-1 / 4+3 \epsilon / 2}\right)\right) .
$$

Hence from the unimodality discussed above,

$$
\sum_{\left(m_{+}, k\right) \notin D} f\left(m_{+}, k\right) \leq n^{2} \max _{\left(m_{+}, k\right) \in \partial D} f\left(m_{+}, k\right) \leq \frac{n^{2}}{\sqrt{e n} \pi}(2 n)^{n / 2} e^{-n / 2+\sqrt{2 n}} e^{-5 \sqrt{2} n^{2 \epsilon}},
$$

and by summing up using (8.31),

$$
\sum_{\left(m_{+}, k\right) \in D} f\left(m_{+}, k\right)=\frac{1}{\sqrt{2 e}}(2 n)^{n / 2} e^{-n / 2+\sqrt{2 n}}\left(1+O\left(n^{-1 / 4+3 \epsilon / 2}\right)\right) .
$$

Hence we have

$$
\left|\tilde{S}_{n}^{\bigotimes}\right|=\frac{1}{\sqrt{2 e}}(2 n)^{n / 2} e^{-n / 2+\sqrt{2 n}}\left(1+O\left(n^{-1 / 4+3 \epsilon / 2}\right)\right),
$$


and the main contribution to the sum in 8.28 ) comes from $D$.

As in Theorem 3.4, we write

$$
\operatorname{Pr}\left(\tilde{L}_{n}^{\bigotimes} \leq l\right)=\frac{1}{\left|\tilde{S}_{n}^{\bigotimes}\right|}\left[\sum_{D} p_{k m_{+} m_{-} l}\left|\tilde{S}_{k, m_{+}, m_{-}}^{\bigotimes}\right|+\sum_{D^{c}} p_{k m_{+} m_{-} l}\left|\tilde{S}_{k, m_{+}, m_{-}}^{\bigotimes}\right|\right] .
$$

From (8.32) and 8.34,

$$
\frac{1}{\left|\tilde{S}_{n}^{凶 \mid}\right|} \sum_{D^{c}} p_{n m_{+} m_{-} l}\left|\tilde{S}_{n, m_{+}, m_{-}}^{\bowtie}\right| \leq e^{-10 n^{2 \epsilon}} .
$$

On the other hand, by the remark to Lemma 6.3 and Theorem 4.1, (recall (4.9)

$$
p_{k m_{+} m_{-} l} \leq e^{-\nu_{m_{+}}^{(d)}-\nu_{k}^{(d)}} \pi_{[l / 2]}^{*}\left(-\frac{\nu_{m_{+}}^{(d)}}{\left(\nu_{k}^{(d)}\right)^{1 / 2}} ;\left(\nu_{k}^{(d)}\right)^{1 / 2}\right) D_{\left[\frac{l}{2}\right]}\left(\left(\nu_{k}^{(d)}\right)^{1 / 2}\right)+C n^{-d / 2},
$$

for large $n$. We have a similar inequality of the other direction with $\nu, l$ and $+C n^{-d / 2}$ replaced by $\mu, l-1$ and $-C n^{-d / 2}$.

Let $l=\left[2 \sqrt{2 n}+x 2^{2 / 3}(2 n)^{1 / 6}\right]$. In the region $D$,

$$
\left(l / 2-4\left(\nu_{k}^{(d)}\right)^{1 / 2}\right)(l / 4)^{-1 / 3}=x+O\left(n^{-1 / 6} \sqrt{\log n}\right),
$$

and

$$
\left(1-\nu_{m_{+}}^{(d)} /\left(\nu_{k}^{(d)}\right)^{1 / 2}\right) 2^{-4 / 3}(l / 2)^{1 / 3}=O\left(n^{-1 / 12+\epsilon}\right) .
$$

Hence as in (8.14), using Corollary 7.2 (7.5), Proposition 5.4 and Corollary 5.5

$$
\begin{array}{r}
\left|e^{-\nu_{m_{+}}^{(d)}-\nu_{k}^{(d)}} \pi_{[l / 2]}^{*}\left(-\frac{\nu_{m_{+}}^{(d)}}{\left(\nu_{k}^{(d)}\right)^{1 / 2}} ;\left(\nu_{k}^{(d)}\right)^{1 / 2}\right) D_{\left[\frac{l}{2}\right]}\left(\left(\nu_{k}^{(d)}\right)^{1 / 2}\right)-F^{凶}(x ; 0)\right| \\
\leq C n^{-1 / 6} \sqrt{\log n}+C n^{-1 / 12+\epsilon}+C(M) n^{-1 / 6}+C e^{-c M^{3 / 2}}
\end{array}
$$

for a constant $C(M)$ which may depend on $M$ and constants $C$ and $c$ which is independent of $M$. Thus for large $n$,

$$
\operatorname{Pr}\left(\frac{\tilde{L}_{n}^{\bigotimes}-2 \sqrt{2 n}}{2^{2 / 3}(2 n)^{1 / 6}} \leq x\right) \leq F^{\bigotimes}(x ; 0)+e(n, M)
$$

with some error $e(n, M)$ such that $\lim _{M \rightarrow \infty} \lim _{n \rightarrow \infty} e(n, M)=0$. Similarly we have an inequality for the other direction. Recalling $F^{\otimes}(x ; 0)=F_{1}(x)^{2}$ from (2.35), we obtain (3.21).

Proof of (3.23). Integrating by parts,

$$
\mathrm{E}\left(\left(\tilde{\chi}_{n}^{\otimes}\right)^{p}\right)=\int_{-\infty}^{\infty} x^{p} d F_{n}(x)=-\int_{-\infty}^{0} p x^{p-1} F_{n}(x) d x+\int_{0}^{\infty} p x^{p-1}\left(1-F_{n}(x)\right) d x,
$$

where $F_{n}(x):=\operatorname{Pr}\left(\tilde{\chi}_{n}^{\bigotimes} \leq x\right)=\operatorname{Pr}\left(\tilde{L}_{n}^{\bigotimes} \leq 2 \sqrt{2 n}+x 2^{2 / 3}(2 n)^{1 / 6}\right)$. Note that when $x<-(4 n)^{1 / 3}, F_{n}(x)=0$, and when $x>2^{1 / 6} n^{5 / 6}-(4 n)^{1 / 3}, F_{n}(x)=1$. 
Let $M>M_{0}$ fixed. Consider the case when $-(4 n)^{1 / 3} \leq x \leq-M$. From (8.35) and (8.36),

$$
F_{n}(x) \leq \frac{1}{\left|\tilde{S}_{n}^{\otimes}\right|} \sum_{D} p_{k m_{+} m_{-} l}\left|\tilde{S}_{k, m_{+}, m_{-}}^{\otimes}\right|+C e^{-10 n^{2 \epsilon}}
$$

where $l=\left[2 \sqrt{2 n}+x 2^{2 / 3}(2 n)^{1 / 6}\right]$. We apply Lemma 6.4, Corollary 7.6 and Corollary 5.7 5.36). Note that we are in the region $\alpha \rightarrow 1$ faster than $k^{-1 / 3}$, hence $w$ is bounded, say $-1 \leq w \leq 1$. So we can apply Corollary 5.7 (5.36). Then we obtain

$$
F_{n}(x) \leq C e^{-c|x|^{3}}+C e^{-10 n^{2 \epsilon}}
$$

Since $-(4 n)^{1 / 3} \leq x \leq-M$, we have

$$
e^{-10 n^{2 \epsilon}} \leq e^{-\frac{10}{2^{4 \epsilon}}|x|^{6 \epsilon}}
$$

thus,

$$
F_{n}(x) \leq C e^{-c|x|^{3}}+C e^{-\frac{10}{2^{4 \epsilon}}|x|^{6 \epsilon}} .
$$

On the other hand, when $M \leq x \leq 2^{1 / 6} n^{5 / 6}-(4 n)^{1 / 3}$, similarly we have

$$
1-F_{n}(x) \leq \frac{1}{\left|\tilde{S}_{n}^{\otimes}\right|} \sum_{D}\left(1-p_{k m_{+} m_{-} l}\right)\left|\tilde{S}_{k, m_{+}, m_{-}}^{\bigotimes}\right|+C e^{-10 n^{2 \epsilon}},
$$

Using Lemma 6.4, Corollary 7.6 and Proposition 5.6 (5.32), we obtain

$$
1-F_{n}(x) \leq C e^{-c|x|^{3 / 2}}+C e^{-10 n^{2 \epsilon}} .
$$

Since $M \leq x \leq 2^{1 / 6} n^{5 / 6}-(4 n)^{1 / 3}$,

$$
e^{-10 n^{2 \epsilon}} \leq e^{-\frac{10}{2^{2 \epsilon}|x|^{12 \epsilon / 5}}}
$$

thus

$$
1-F_{n}(x) \leq C e^{-c|x|^{3 / 2}}+C e^{-\frac{10}{2^{2 \epsilon / 5}}|x|^{12 \epsilon / 5}}
$$

Therefore, using dominated convergence theorem, we obtain (3.23).

\section{$9 \quad$ Asymptotics for $\alpha>1$}

As we remarked after Theorem 3.3, when $\alpha>1$, we must use a different scaling to obtain useful results.

Let $L^{\square}(t ; \alpha)$ and $L^{\varpi}(t ; \alpha, \beta)$ be random variables with the distribution functions given by $\operatorname{Pr}\left(L^{\square}(t ; \alpha) \leq\right.$ $l)=P_{l}^{\square}(t ; \alpha)$ and $\operatorname{Pr}\left(L^{\bigotimes}(t ; \alpha, \beta) \leq l\right)=P_{l}^{\bigotimes}(t ; \alpha, \beta)$, respectively: the Poissonized version of $L^{\square}$ and $L^{\bigotimes}$. Under appropriate scalings, we obtain the Gaussian distribution in the limit. 
Theorem 9.1. For $\alpha>1$ and $\beta \geq 0$ fixed,

$$
\begin{aligned}
\lim _{t \rightarrow \infty} \operatorname{Pr}\left(\frac{L^{\square}(t ; \alpha)-\left(\alpha+\alpha^{-1}\right) t}{\sqrt{\left(\alpha-\alpha^{-1}\right) t}} \leq x\right) & =\frac{1}{\sqrt{2 \pi}} \int_{-\infty}^{x} e^{-\frac{1}{2} y^{2}} d y, \\
\lim _{t \rightarrow \infty} \operatorname{Pr}\left(\frac{L^{\bigotimes}(t ; \alpha, \beta)-2\left(\alpha+\alpha^{-1}\right) t}{\sqrt{2\left(\alpha-\alpha^{-1}\right) t}} \leq x\right) & =\frac{1}{\sqrt{2 \pi}} \int_{-\infty}^{x} e^{-\frac{1}{2} y^{2}} d y .
\end{aligned}
$$

Proof. Let $l=\left(\alpha+\alpha^{-1}\right) t+\sqrt{\left(\alpha-\alpha^{-1}\right) t}$ for $L^{\square}$. For large $t, 2 t / l \leq c<1$ for some $c>0$. From Proposition 5.1 (i), using Theorem 4.1, it is easy to see that $e^{-t^{2} / 2} D_{l}^{ \pm \pm}(t), e^{-t^{2} \pm t} D_{l}^{ \pm \mp}(t) \rightarrow 1$ exponentially as $l \rightarrow \infty$. Now Theorem 4.1, (5.29) and Proposition 5.8 imply (9.1). For $L^{\bigotimes}$, let $l=2\left(\alpha+\alpha^{-1}\right) t+\sqrt{2\left(\alpha-\alpha^{-1}\right) t}$. Similarly, $e^{-t^{2}} D_{l}(t) \rightarrow 1$ exponentially as $l \rightarrow \infty$, and we obtain (9.2).

Unfortunately, we can no longer apply the de-Poissonization technique; the difficulty is that $\left(\alpha+\alpha^{-1}\right) t$ depends too strongly on small perturbations in $\alpha$. Indeed, as we shall see, the asymptotics of the non-Poisson processes are different.

Consider the case of involutions with $[\alpha t]$ fixed points and $\left[t^{2} / 2\right] 2$-cycles; the case of signed involutions is analogous. By symmetry, this is the same as the largest increasing subset distribution for the point selection process in the triangle $0 \leq y \leq x \leq 1$ with $\left[t^{2} / 2\right]$ generic points and $[\alpha t]$ diagonal points. As was observed in Remark 2 to Corollary 7.6 of [7], it is equivalent to consider weakly increasing subsets where the extra points are added to the line $y=0$ instead of to the diagonal.

As in (3.1), let

$$
\chi_{\left[t^{2} / 2\right],[\alpha t]}^{\square}=\frac{L_{\left[t^{2} / 2\right],[\alpha t]}^{\square}-(\alpha+1 / \alpha) t}{\sqrt{\left(1 / \alpha-1 / \alpha^{3}\right) t}} .
$$

Theorem 9.2. As $t \rightarrow \infty$, the variable $\chi_{\left[t^{2} / 2\right],[\alpha t]}^{\square}$ converges in distribution and moments to $N(0,1)$.

Proof. Let $S(t)$ be the set of points at time $t$, and let $I$ be a largest increasing subset of $S(t)$. Then there will exist some number $0 \leq s^{+} \leq 1$ (not unique) such that

$$
\left(S(t) \cap\left\{y=0,0 \leq x \leq s^{+}\right\}\right) \subset I,
$$

and such that every other point of $I$ has $x>s^{+}$and $y>0$. For any $0 \leq s \leq 1$, we thus have

$$
f_{1}(s)+f_{2}(s) \leq|I|,
$$

where $f_{1}(s)$ is the number of points of $S(t)$ with $y=0$ and $0 \leq x \leq s$, and where $f_{2}(s)$ is the largest increasing subset of $S(t)$ lying entirely in the (part-open) trapezoid with $x \geq s, y>0$.

Since $f_{1}(s)$ is binomial with parameters $[\alpha t]$ and $s$,

Lemma 9.3. Let $M>0$ be sufficiently large and fixed. For all $0 \leq s<1$, there exist positive constants $C, c$ independent of $s$ such that for $w \geq M$,

$$
\operatorname{Pr}\left(f_{1}(s)>\alpha s t+w t^{1 / 2}\right) \leq C e^{-c|w|^{2}}
$$

while for $w \leq-M$,

$$
\operatorname{Pr}\left(f_{1}(s)<\alpha s t+w t^{1 / 2}\right) \leq C e^{-c|w|^{2}} .
$$


For $f_{2}$, we have:

Lemma 9.4. Let $M>0$ be sufficiently large and fixed. For $0 \leq s<1$, there exist positive constants $C$ and $c$ independent of $s$ such that for all $w \geq M$,

$$
\operatorname{Pr}\left(f_{2}(s)>2 \sqrt{(1-s)} t+w t^{1 / 3}\right) \leq C e^{-c|w|^{3 / 2}},
$$

and for all $w \leq-M$,

$$
\operatorname{Pr}\left(f_{2}(s)<2 \sqrt{(1-s)} t+w t^{1 / 3}\right) \leq C e^{-c|w|^{3}} .
$$

Proof. We first show the corresponding large-deviation result for the Poissonization. Define $f_{2}^{\prime}(s, t)$ to be the length of the longest increasing subsequence when the number of points in the trapezoid is Poisson with parameter $t^{2}\left(1-s^{2}\right) / 2$. Then $f_{2}^{\prime}(s, t)$ is bounded between the corresponding processes for the rectangle $s \leq x \leq 1$, $0 \leq y \leq 1$ and for the triangle $0 \leq(x-s) /(1-s) \leq y \leq 1$. In particular, if $f_{2}^{\prime}(s, t)$ deviates significantly from $\sqrt{1-s} t$, so must the appropriate bounding process; the result follows immediately from the corresponding results for rectangles and triangles.

The corresponding large-deviation result when the number of points is fixed then follows from Lemma 6.2. In our case, the number of points in the trapezoid is binomial with parameters $t^{2} / 2$ and $\left(1-s^{2}\right)$; the lemma follows via essentially the same argument used to prove Lemma 6.2.

As we will see, the value $s=1-\alpha^{-2}$ deserves special attention:

Lemma 9.5. Let $M>0$ be sufficiently large and fixed. There exist positive constants $C$, $c$ such that for $w \geq M$,

$$
\operatorname{Pr}\left(f_{1}\left(1-\alpha^{-2}\right)+f_{2}\left(1-\alpha^{-2}\right)>(\alpha+1 / \alpha) t+w t^{1 / 2}\right) \leq C e^{-c \min \left(|w|^{2},|w|^{3 / 2} t^{1 / 4}\right)},
$$

and for $w \leq-M$,

$$
\operatorname{Pr}\left(f_{1}\left(1-\alpha^{-2}\right)+f_{2}\left(1-\alpha^{-2}\right)<(\alpha+1 / \alpha) t+w t^{1 / 2}\right) \leq C e^{-c|w|^{2}} .
$$

Moreover, if we define

$$
\chi_{0}(t)=\frac{f_{1}\left(1-\alpha^{-2}\right)+f_{2}\left(1-\alpha^{-2}\right)-(\alpha+1 / \alpha) t}{\sqrt{\left(1 / \alpha-1 / \alpha^{3}\right) t}},
$$

then $\chi_{0}(t)$ converges to a standard normal distribution, both in distribution and in moments.

Proof. That $\chi_{0}(t)$ converges as stated follows from the fact that if we write $\chi_{0}(t)=\chi_{1}(t)+\chi_{2}(t)$, with

$$
\begin{aligned}
\chi_{1}(t) & =\frac{f_{1}\left(1-\alpha^{-2}\right)-(\alpha-1 / \alpha) t}{\sqrt{\left(1 / \alpha-1 / \alpha^{3}\right) t}} \\
\chi_{2}(t) & =\frac{f_{2}\left(1-\alpha^{-2}\right)-(2 / \alpha) t}{\sqrt{\left(1 / \alpha-1 / \alpha^{3}\right) t}},
\end{aligned}
$$

then $\chi_{1}(t)$ converges in distribution and moments to a standard normal distribution, and $\chi_{2}(t)$ converges in distribution and moments to 0 . 
For the large deviation bounds, we note that if $x+y>z+w$, then either $x>z$ or $y>w$. Thus for any $0 \leq b \leq 1$, we have

$$
\begin{aligned}
\operatorname{Pr}\left(f_{1}\left(1-\alpha^{-2}\right)\right. & \left.+f_{2}\left(1-\alpha^{-2}\right)>(\alpha+1 / \alpha) t+w t^{1 / 2}\right) \\
\leq & \operatorname{Pr}\left(f_{1}\left(1-\alpha^{-2}\right)>(\alpha-1 / \alpha) t+b w t^{1 / 2}\right)+\operatorname{Pr}\left(f_{2}\left(1-\alpha^{-2}\right)>(2 / \alpha) t+(1-b) w t^{1 / 2}\right) \\
\leq & C e^{-c|b w|^{2}}+C e^{-c|(1-b) w|^{3 / 2} t^{1 / 4}}
\end{aligned}
$$

the result follows by balancing the two terms. In the other case, the $C e^{-c|w|^{2}}$ term always dominates.

Lemma 9.6. For any sufficiently small $\epsilon>0$, there exist positive constants $C, c$ such that

$$
\operatorname{Pr}\left(s^{+}-\left(1-1 / \alpha^{2}\right)>t^{\epsilon / 3-1 / 3}\right)<C e^{-c t^{\epsilon}}
$$

and

$$
\operatorname{Pr}\left(\left(1-1 / \alpha^{2}\right)-s^{+}>t^{\epsilon / 2-1 / 2}\right)<C e^{-c t^{\epsilon}}
$$

for all sufficiently large $t$.

Proof. Define a sequence $s_{i}$ by taking

$$
s_{i}=1-(1-2 /(i+2))^{2} / \alpha^{2}
$$

for all $i \geq 0$. Similarly define a sequence $s_{i}^{\prime}$ by

$$
s_{i}^{\prime}=\max \left(t_{i}, 0\right),
$$

with

$$
t_{i}=1-\left(1+2 e^{2^{-1-i}}\right)^{2} / \alpha^{2}
$$

for $i<0$ and

$$
t_{i}=1-(1+4 /(i+1))^{2} / \alpha^{2}
$$

for $i \geq 0$. Note that $s_{i}$ is strictly decreasing and $t_{i}$ is strictly increasing.

Lemma 9.7. For all $i \geq 0$,

$$
\alpha s_{i}+2 \sqrt{1-s_{i+1}}<\alpha+1 / \alpha .
$$

For all $i$,

$$
\alpha s_{i+1}^{\prime}+2 \sqrt{1-s_{i}^{\prime}}<\alpha+1 / \alpha .
$$


Proof. In the first case, we have

$$
\alpha+1 / \alpha-\left(\alpha s_{i}+2 \sqrt{1-s_{i+1}}\right)=\frac{4}{(i+2)^{2}(i+3) \alpha} .
$$

In the second case, it suffices to verify the formula with $s^{\prime}$ replaced by $t$. For $i<-1$,

$$
\alpha+1 / \alpha-\left(\alpha t_{i+1}+2 \sqrt{1-t_{i}}\right)=4 e^{2^{-i} / 4} / \alpha .
$$

For $i=-1$,

$$
\alpha+1 / \alpha-\left(\alpha t_{i+1}+2 \sqrt{1-t_{i}}\right)=(24-4 e) / \alpha
$$

Finally, for $i \geq 0$,

$$
\alpha+1 / \alpha-\left(\alpha t_{i+1}+2 \sqrt{1-t_{i}}\right)=\frac{8 i}{(i+1)(i+2)^{2} \alpha} .
$$

Let $i_{1}=t^{1 / 6-\epsilon / 6}, i_{2}=t^{1 / 4-\epsilon / 4}$. Then there exist constants $C, c$ such that for $0 \leq i \leq i_{1}$,

$$
\operatorname{Pr}\left(f_{1}\left(s_{i}\right)+f_{2}\left(s_{i+1}\right)>f_{1}\left(1-\alpha^{-2}\right)+f_{2}\left(1-\alpha^{-2}\right)\right)<C e^{-c t^{\epsilon}} .
$$

Since $f_{1}\left(s_{i}\right)+f_{2}\left(s_{i+1}\right)$ is an upper bound on $f_{1}(s)+f_{2}(s)$ with $s_{i+1} \leq s \leq s_{i}$, it follows that

$$
\operatorname{Pr}\left(s^{+} \in\left[s_{i+1}, s_{i}\right]\right)<C e^{-c t^{\epsilon}} .
$$

Similarly, for $i \leq i_{2}$,

$$
\operatorname{Pr}\left(f_{1}\left(s_{i+1}^{\prime}\right)+f_{2}\left(s_{i}^{\prime}\right)>f_{1}\left(1-\alpha^{-2}\right)+f_{2}\left(1-\alpha^{-2}\right)\right)<C e^{-c t^{\epsilon}},
$$

and thus

$$
\operatorname{Pr}\left(s^{+} \in\left[s_{i}^{\prime}, s_{i+1}^{\prime}\right]\right)<C e^{-c t^{\epsilon}} .
$$

Since there are only $i_{1}+i_{2}+\log \log \alpha$ such events to consider, the result follows.

In particular, with probability $1-C e^{-c t^{\epsilon}}$, we have

$$
f_{1}\left(1-\alpha^{-2}-t^{\epsilon / 2-1 / 2}\right)+f_{2}\left(1-\alpha^{-2}+t^{\epsilon / 3-1 / 3}\right) \leq L(t) \leq f_{1}\left(1-\alpha^{-2}+t^{\epsilon / 3-1 / 3}\right)+f_{2}\left(1-\alpha^{-2}-t^{\epsilon / 2-1 / 2}\right) .
$$

But then using the fact that

$$
f_{1}\left(1-\alpha^{-2}\right)-f_{1}\left(1-\alpha^{-2}-t^{\epsilon / 2-1 / 2}\right)
$$

and

$$
f_{1}\left(1-\alpha^{-2}-t^{\epsilon / 2-1 / 2}\right)-f_{1}\left(1-\alpha^{-2}\right)
$$


are Poisson, and using the large deviation behavior of $f_{2}(s)$, we find that

$$
\operatorname{Pr}\left(L(t)-\left(f_{1}\left(1-\alpha^{-2}-t^{\epsilon / 2-1 / 2}\right)+f_{2}\left(1-\alpha^{-2}+t^{\epsilon / 3-1 / 3}\right)\right) \geq t^{1 / 2-\epsilon^{\prime}}\right) \leq C e^{-c t^{\epsilon}},
$$

and

$$
\operatorname{Pr}\left(\left(f_{1}\left(1-\alpha^{-2}-t^{\epsilon / 3-1 / 3}\right)+f_{2}\left(1-\alpha^{-2}+t^{\epsilon / 2-1 / 2}\right)\right)-L(t) \geq t^{1 / 2-\epsilon^{\prime}}\right) \leq C e^{-c t^{\epsilon}} .
$$

So $\chi(t)-\chi_{0}(t)$ converges to 0 in a fairly strong sense; in particular, they must have the same limiting distribution and limiting moments.

Remark. The above proof could equally well be applied to the Poisson process; the beta distribution would then be replaced by a Poisson distribution.

For the signed involution case, with $[2 \alpha t]$ fixed points, $[2 \beta t]$ negated points, and $\left[2 t^{2}\right] 2$-cycles, again we let

$$
\chi_{\left[t^{2}\right],[2 \alpha t],[2 \beta t]}^{\bigotimes}=\frac{L_{\left[t^{2} / 2\right],[\alpha t],[\beta t]}^{\bigotimes}-2(\alpha+1 / \alpha) t}{\sqrt{2\left(1 / \alpha-1 / \alpha^{3}\right) t}} .
$$

Then the analogous argument proves that $\chi^{\prime}(t)$ also converges in moments and distribution to a standard normal.

\section{Steepest descent type analysis for Riemann-Hilbert problems}

In this section, we prove the asymptotics of orthogonal polynomials results stated in Section 5 by applying the steepest descent type method to the Riemann-Hilbert problem (RHP) (5.4). The steepest descent method for RHP's, the Deift-Zhou method, was introduced by Deift and Zhou in [18] and is developed further in [19], [16], and finally placed in a systematic form by Deift, Venakides and Zhou in [17]. The steepest descent analysis of the RHP (5.4) was first conducted in [3]. The analysis of [3] has many similarities with [13, 14, 15] where the asymptotics of orthogonal polynomials on the real line with respect to a general weight is obtained, leading to a proof of universality conjectures in random matrix theory.

As mentioned in the Introduction and Section 5, in this section we extend the analysis of [3] and obtain new estimates on the orthogonal polynomials $\pi_{k}(z ; t)$. The extension is done roughly in two categories. In [3], the quantity of interest was $Y_{21}(0 ; k ; t)$, and so the $z$-dependence of the error bound of $Y(z ; k ; t)$ was not considered carefully. But in the present paper we need the asymptotics of $Y(z)$ for general $z \in \mathbb{C}$, and also for the case when $z \rightarrow-1$ as $k, t \rightarrow \infty$. Hence the first category of our extension is to investigate how the error estimate depends on $z$. This task sometimes requires improved estimates of the solution $Y(z)$. See for example, (10.42) below where an improved $L^{1}$ norm bound of the jump matrix is needed. On the other hand, as we will see, the asymptotic solution $Y(z)$ is expressed in terms of the so-called the $g$-function. Thus we need detailed analysis of the $g$-function to obtain the asymptotics of the orthogonal polynomials. In the special case $z=0$, we have $g(0)=\pi i$ (Lemma 4.2 in [3]). Hence in [3], the analysis of the $g$-function was quite simple. But in the present paper, we need general values of $g(z)$, and this in some cases requires further analysis. Hence the analysis of 
the $g$-function is the second category of our extension. For example, see 110.109)-(10.121) below where we need a further analysis of the $g$-function.

Again the analysis in this section relies heavily on the analysis of [3] and we extend the method of [3]. Thus the analysis below overlaps in many parts with the analysis of [3]. However, for continuity of presentation and also for the convenience of readers, we include some calculations which overlap [3]. When the analysis overlaps that of [3], we only sketch the method, and instead we focus on new features to indicate how to prove the propositions in Section 5 .

We say that a RHP is normalized at $\infty$ if the solution $m$ satisfies the condition $m \rightarrow I$ as $z \rightarrow \infty$. Thus for instance, the RHP's (2.15), (10.1) are normalized at $\infty$, while the RHP (5.4) is not.

In 3, it turned out that the asymptotic analysis differs critically when $(2 t) / k \leq 1$ and $(2 t) / k>1$, due to the difference of (the support of) the associated equilibrium measure (see Lemma 4.3 [3]). Hence we discuss those two cases separately in Subsections 10.1 and 10.2, which extend Section 5 and Section 6 of [3], respectively. Each section is also divided into three subcases. In each subcase the corresponding case of the propositions in Section (except Proposition 5.8) are proved. Subsection 10.3 is new, and Proposition 5.8 is proved in this subsection.

\subsection{When $(2 t) / k \leq 1$.}

The following algebraic transformations (10.1)-(10.10) of RHP's are taken from (5.1)-(5.3) of [3].

Define

$$
\begin{array}{cc}
m^{(1)}(z ; k ; t):=Y(z ; k ; t)\left(\begin{array}{cc}
(-1)^{k} e^{t z} & 0 \\
0 & (-1)^{k} e^{-t z}
\end{array}\right), & |z|<1 \\
m^{(1)}(z ; k ; t):=Y(z ; k ; t)\left(\begin{array}{cc}
z^{-k} e^{t z^{-1}} & 0 \\
0 & z^{k} e^{-t z^{-1}}
\end{array}\right), & |z|>1 .
\end{array}
$$

Then $m^{(1)}$ solves a new RHP which is equivalent to the RHP (5.4) in the sense that a solution of one RHP yields algebraically a solution of the other RHP, and vice versa:

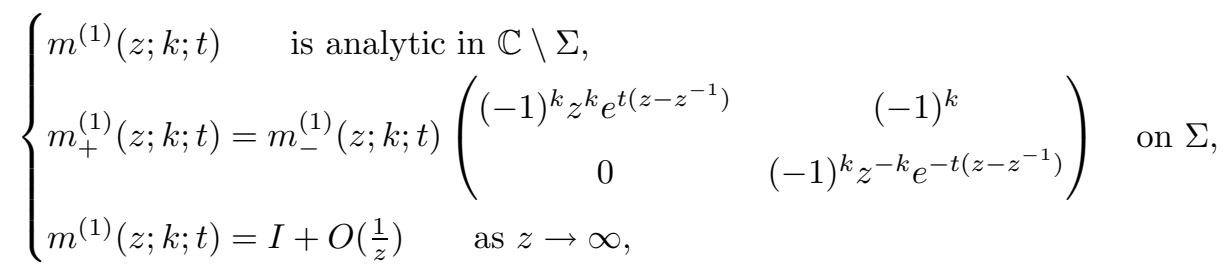

where $\Sigma$ is the unit circle oriented counterclockwise as before. Here and in the sequel, $m_{+}(z)$ (resp. $\left.m_{-}(z)\right)$ is understood as the limit from the left (reps. right) side of the contour as one goes along the orientation of the 
contour. Now we define $m^{(2)}(z ; k ; t)$ in terms of $m^{(1)}(z ; k ; t)$ as follows:

for even $k$,

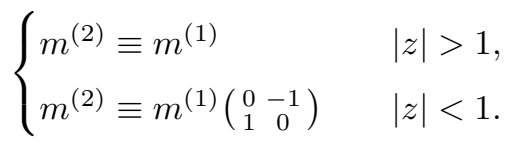

for odd $k$,

$$
\begin{cases}m^{(2)} \equiv\left(\begin{array}{cc}
1 & 0 \\
0 & -1
\end{array}\right) m^{(1)}\left(\begin{array}{cc}
1 & 0 \\
0 & -1
\end{array}\right) & |z|>1 \\
m^{(2)} \equiv\left(\begin{array}{cc}
1 & 0 \\
0 & -1
\end{array}\right) m^{(1)}\left(\begin{array}{cc}
0 & -1 \\
-1 & 0
\end{array}\right) & |z|<1\end{cases}
$$

Then $m^{(2)}(\cdot ; k ; t)$ solves another RHP

$$
\left\{\begin{array}{l}
m_{+}^{(2)}(z ; k ; t)=m_{-}^{(2)}(z ; k ; t) v^{(2)}(z ; k ; t) \text { on } \Sigma \\
m^{(2)}(z ; k ; t)=I+O\left(\frac{1}{z}\right) \text { as } z \rightarrow \infty
\end{array}\right.
$$

where

$$
v^{(2)}(z ; k ; t)=\left(\begin{array}{cc}
1 & -(-1)^{k} z^{k} e^{t\left(z-z^{-1}\right)} \\
(-1)^{k} z^{-k} e^{-t\left(z-z^{-1}\right)} & 0
\end{array}\right) .
$$

The jump matrix has the following factorization,

$$
v^{(2)}=\left(\begin{array}{cc}
1 & 0 \\
(-1)^{k} z^{-k} e^{-t\left(z-z^{-1}\right)} & 1
\end{array}\right)\left(\begin{array}{cc}
1 & -(-1)^{k} z^{k} e^{t\left(z-z^{-1}\right)} \\
0 & 1
\end{array}\right)=:\left(b_{-}^{(2)}\right)^{-1} b_{+}^{(2)},
$$

We note that through the changes $Y \rightarrow m^{(1)} \rightarrow m^{(2)}$, we have

$$
\begin{aligned}
Y_{11}(z ; k ; t) & =-(-1)^{k} e^{-t z} m_{12}^{(2)}(z ; k ; t), & & |z|<1, \\
Y_{21}(z ; k ; t) & =-e^{-t z} m_{22}^{(2)}(z ; k ; t), & & |z|<1, \\
Y_{11}(z ; k ; t) & =z^{k} e^{-t z^{-1}} m_{11}^{(2)}(z ; k ; t), & & |z|>1, \\
Y_{21}(z ; k ; t) & =(-z)^{k} e^{-t z^{-1}} m_{21}^{(2)}(z ; k ; t), & & |z|>1 .
\end{aligned}
$$

As in (5.4) of [3], the absolute value of the (12)-entry of the jump matrix $v^{(2)}$ is $e^{k F\left(\rho, \theta ; \frac{2 t}{k}\right)}$ where

$$
F(z ; \gamma)=F\left(\rho e^{i \theta} ; \gamma\right):=\frac{\gamma}{2}\left(\rho-\rho^{-1}\right) \cos \theta+\log \rho, \quad z=\rho e^{i \theta}
$$

The absolute value of the $(21)$-entry of $v^{(2)}$ is $e^{-k F\left(\rho e^{i \theta} ; \frac{2 t}{k}\right)}$. Note that

$$
F(\rho, \theta ; \gamma)=-F\left(\rho^{-1}, \theta ; \gamma\right)
$$

Figure 3 shows the curves $F(z ; \gamma)=0$. In $\Omega_{1}^{\text {sig }} \cup \Omega_{3}^{\text {sig }}, F>0$, and in $\Omega_{2}^{\text {sig }} \cup \Omega_{4}^{\text {sig }}, F<0$. The region $\Omega_{2}^{\text {sig }}$ becomes smaller as $\gamma$ increases, and when $\gamma=1$, the curve $F(z ; \gamma)=0$ contacts the unit circle $\Sigma$ at $z=-1$ with the angle $\pi / 3$.

We distinguish three subcases as in Section 5 of [3]. 


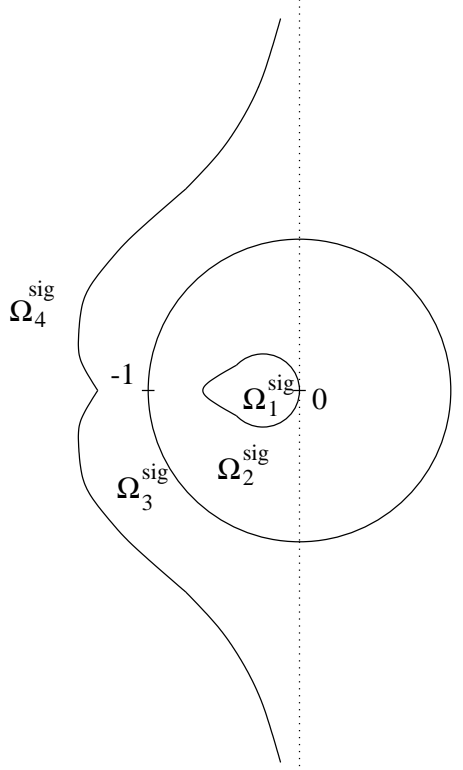

Figure 3: curves of $F(z ; \gamma)=0$ when $0<\gamma<1$

10.1.1 The case $0 \leq 2 t \leq a k$ for some $0<a<1$.

It is possible to fix $\rho_{a}<1$ such that the circle $\left\{z:|z|=\rho_{a}\right\}$ is in the region $\Omega_{2}^{\text {sig }}$ for all such $t$ and $k$. Define $m^{(3)}(z ; k ; t)$ by (see $(5.9)$ of [3])

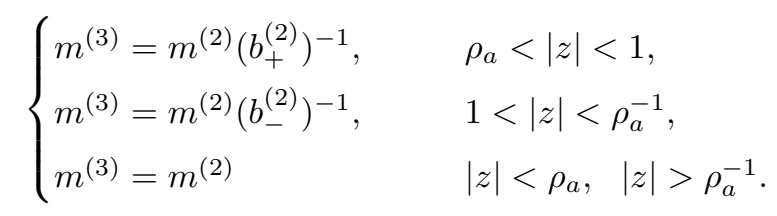

Then $m^{(3)}$ satisfies a new jump condition $m_{+}^{(3)}=m_{-}^{(3)} v^{(3)}$ on $\Sigma^{(3)}:=\left\{z:|z|=\rho_{a}, \rho_{a}^{-1}\right\}$, where $v^{(3)}=b_{+}^{(2)}$, $|z|=\rho_{a}$ and $v^{(3)}=\left(b_{-}^{(2)}\right)^{-1},|z|=\rho_{a}^{-1}$. This $\Sigma^{(3)}$ is not the best choice (see the next subsubsection). But for a simple and direct estimate, we use this choice in this subsubsection. From the choice of $\rho_{a}$, we have (see $(5.13)-(5.14)$ of [3])

$$
\left|v^{(3)}(z ; k ; t)-I\right| \leq e^{-c k} \quad \text { for all } z \in \Sigma^{(3)},
$$

which implies that $I-C_{w^{(3)}}$ is invertible and the norm of the inverse is uniformly bounded, where $w^{(3)}:=v^{(3)}-I$ and $C_{w^{(3)}}(f):=C_{-}\left(f w^{(3)}\right)$ on $L^{2}\left(\Sigma^{(3)},|d z|\right), C_{ \pm}$being Cauchy operators (see (2.5)-(2.9) in [3] and references therein). From the general theory of RHP's, we have

$$
m^{(3)}(z)=I+\frac{1}{2 \pi i} \int_{\Sigma^{(3)}} \frac{\left(\left(I-C_{w^{(3)}}\right)^{-1} I\right)(s) w^{(3)}(s)}{s-z} d s, \quad z \notin \Sigma^{(3)} .
$$

This implies the estimates (see (5.16) of [3])

$$
\left|m_{22}^{(3)}(0 ; k ; t)-1\right|, \quad\left|m_{12}^{(3)}(0 ; k ; t)\right| \leq C e^{-c k},
$$


which using (10.13), 10.7), (10.8) and (5.5), (5.6), yield Proposition 5.1 (i). This is precisely the result contained in $(5.17)$ of [3].

From (10.13), 10.7), (10.9) and (5.6), we have

$$
\begin{array}{lll}
\pi_{k}(z ; t)=-(-1)^{k} e^{-t z} m_{12}^{(3)}(z ; k ; t), & |z|<\rho_{a}, \\
\pi_{k}(z ; t)=z^{k} e^{-t z^{-1}} m_{11}^{(3)}(z ; k ; t), & |z|>\rho_{a}^{-1}, \\
\pi_{k}(z ; t)=z^{k} e^{-t z^{-1}} m_{11}^{(3)}(z ; k ; t)-(-1)^{k} e^{-t z} m_{12}^{(3)}(z ; k ; t), & \rho_{a}<|z|<\rho_{a}^{-1} .
\end{array}
$$

Let $0<b<1$ be a fixed number. From Figure 3, we could have chosen $\rho_{a}$ such that $\rho_{a}>b$. When $|z| \leq b$ and $|z| \geq b^{-1}$, we have $\operatorname{dist}\left(z, \Sigma^{(3)}\right) \geq c>0$. Since $z$ is uniformly bounded away from the contour, we can extend the argument leading to (5.17) in [3] where the uniform boundedness of 0 from the contour is used. Hence using (10.15), 10.17) and (10.18) imply that

$$
\begin{aligned}
\left|e^{t z} \pi_{k}(z ; t)\right| & \leq C e^{-c k}, & & |z| \leq b, \\
\left|e^{t z^{-1}} z^{-k} \pi_{k}(z ; t)\right| & \leq C e^{-c k}, & & |z| \geq b^{-1} .
\end{aligned}
$$

These are 5.29), (5.30) in Proposition 5.6 of the special case $x \geq 2^{1 / 3}(1-a) k^{2 / 3}$.

On the other hand, let $L>0$ be a fixed number. Set $\alpha=1-2^{4 / 3} k^{-1 / 3} w$ with $-L \leq w \leq L$ as in Proposition 5.6. Since $\rho_{a}$ is fixed, when $k$ is large, $\operatorname{dist}\left(-\alpha, \Sigma^{(3)}\right) \geq c>0$. Then from (10.15),

$$
\left|m_{11}^{(3)}(-\alpha ; k ; t)-1\right|, \quad\left|m_{12}^{(3)}(-\alpha ; k ; t)\right| \leq C e^{-c k} .
$$

Note that

$$
\begin{aligned}
\frac{1}{2}\left(s-s^{-1}\right) & \leq s-1, \quad s>0 \\
-\frac{1}{2}\left(s-s^{-1}\right)+\log s & \leq \frac{2}{3}(1-s)^{3}, \quad \frac{1}{2} \leq s \leq 1, \\
-\frac{1}{2}\left(s-s^{-1}\right)+\log s & \leq 0, \quad s \geq 1 .
\end{aligned}
$$

Thus for $\gamma \leq 1, s \geq \frac{1}{2}$,

$$
\begin{aligned}
F(-s ; \gamma) & =-\frac{\gamma}{2}\left(s-s^{-1}\right)+\log s=\frac{1-\gamma}{2}\left(s-s^{-1}\right)-\frac{1}{2}\left(s-s^{-1}\right)+\log s \\
& \leq(1-\gamma)(s-1)+\frac{2}{3}|s-1|^{3} .
\end{aligned}
$$

For large $k, \alpha \geq \frac{1}{2}$ for all $-L \leq w \leq L$, and hence

$$
\left|(-\alpha)^{k} e^{-t\left(\alpha-\alpha^{-1}\right)}\right|=e^{k F\left(-\alpha ; \frac{2 t}{k}\right)} \leq e^{\frac{32}{2}|w|^{3}-k\left(1-\frac{2 t}{k}\right) \frac{2^{4 / 3} w}{k^{1 / 3}}} \leq e^{\frac{32}{2} L^{3}} e^{2^{4 / 3} L k^{2 / 3}}=C e^{c k^{2 / 3}} .
$$

Similarly, since $\alpha^{-1} \geq \frac{1}{2}$ for large $k$,

$$
\left|(-\alpha)^{-k} e^{t\left(\alpha-\alpha^{-1}\right)}\right|=e^{k F\left(-\alpha^{-1} ; \frac{2 t}{k}\right)} \leq e^{k\left(1-\frac{2 t}{k}\right) \frac{2^{4 / 3} w}{k^{1 / 3} \alpha}+\frac{32}{2}|w|^{3} \alpha^{-3}} \leq C e^{c k^{2 / 3}} .
$$


Therefore from (10.19) and (10.22),

$$
\begin{aligned}
\left|e^{-t \alpha} \pi_{k}(-\alpha ; t)\right|=\left|(-\alpha)^{k} e^{-t\left(\alpha-\alpha^{-1}\right)} m_{11}^{(3)}(-\alpha ; k ; t)-(-1)^{k} m_{12}^{(3)}(-\alpha ; k ; t)\right| & \leq C e^{c k^{2 / 3}}, \\
\left|e^{-t \alpha^{-1}}(-\alpha)^{-k} \pi_{k}(-\alpha ; t)-1\right|=\left|m_{11}^{(3)}(-\alpha ; k ; t)-1-\alpha^{-k} e^{t\left(\alpha-\alpha^{-1}\right)} m_{12}^{(3)}(-\alpha ; k ; t)\right| & \leq C e^{-c k} .
\end{aligned}
$$

Noting $x \sim k^{2 / 3}$, these are (5.31), (5.32) in Proposition 5.6 of the special case $x \geq 2^{1 / 3}(1-a) k^{2 / 3}$. Thus we have extended the argument of (5.17) of [3] to the case when $z \rightarrow-1$. This is an example of the extension of the first category mentioned at the beginning of Section 10 (though it is straightforward to extend in this case).

10.1.2 The case $a k \leq 2 t \leq k-M 2^{-1 / 3} k^{1 / 3}$ for some $0<a<1$ and $M>M_{0}$.

In the previous section, the contour $\Omega^{(3)}$ was not the best choice. We could have chosen the steepest descent curve for $F(z ; \gamma)$. For the previous case, it was not necessary to use the steepest descent curve to obtain the desired results, but for the case at hand and in the future calculations, we need to use the steepest descent curve.

For fixed $\theta$ satisfying $0 \leq \theta<\pi / 2$ or $(3 \pi) / 2<\theta<2 \pi, F(\rho, \theta ; \gamma)$ is always negative for $0<\rho<1$, and as $\rho \downarrow 0$, it decreases to minus infinity. On the other hand, one can check that (see (5.5) in [3]) when $\gamma \leq 1$, the minimum of $F(\rho, \theta ; \gamma), 0<\rho \leq 1$ is attained, for fixed $\pi / 2 \leq \theta \leq(3 \pi) / 2$, at

$$
\rho=\rho_{\theta}:=\frac{1-\sqrt{1-\gamma^{2} \cos ^{2} \theta}}{-\gamma \cos \theta}
$$

and $F\left(\rho_{\theta}, \theta ; \gamma\right)<0$. And it is straightforward to check that for $0 \leq \gamma \leq 1, \pi / 2 \leq \theta \leq 3 \pi / 2$,

$$
F\left(\rho_{\theta}, \theta ; \gamma\right)=\sqrt{1-\gamma^{2} \cos ^{2} \theta}+\log \left[\frac{1-\sqrt{1-\gamma^{2} \cos ^{2} \theta}}{-\gamma \cos \theta}\right] \leq-\frac{2 \sqrt{2}}{3}(1+\gamma \cos \theta)^{3 / 2} .
$$

This is an extension of (5.13) in [3] where only the case $\theta=\pi$ was considered. We need this improved version to obtain a better $L^{1}$ estimate of $v^{(3)}-I$ in the sequel. Also $F\left(\rho_{\theta}, \theta ; \gamma\right)$ is increasing in $\pi / 2 \leq \theta \leq \pi$ and is decreasing in $\pi \leq \theta \leq 3 \pi / 2$. In fact, the saddle points for $\frac{\gamma}{2}\left(z-z^{-1}\right)+\log z$ are $z=-\rho_{\pi}$ and $z=-\rho_{\pi}^{-1}$.

In this time, define $\Sigma^{(3)}:=\Sigma_{\text {in }}^{(3)} \cup \Sigma_{\text {out }}^{(3)}$ by, as in (5.6) of [3],

$$
\begin{aligned}
& \Sigma_{\text {in }}^{(3)}=\left\{\rho_{\theta} e^{i \theta}: 3 \pi / 4 \leq \theta \leq 5 \pi / 4\right\} \cup\left\{\rho_{3 \pi / 4} e^{i \theta}: 0 \leq \theta \leq 3 \pi / 4,5 \pi / 4 \leq \theta<2 \pi\right\}, \\
& \Sigma_{\text {out }}^{(3)}=\left\{\rho_{\theta}^{-1} e^{i \theta}: 3 \pi / 4 \leq \theta \leq 5 \pi / 4\right\} \cup\left\{\rho_{3 \pi / 4}^{-1} e^{i \theta}: 0 \leq \theta \leq 3 \pi / 4,5 \pi / 4 \leq \theta<2 \pi\right\},
\end{aligned}
$$

where $\rho_{\theta}$ is defined in (10.31) with $\gamma=(2 t) / k$. Orient $\Sigma^{(3)}$ as in Figure 4 . Note that $\Sigma^{(3)}$ lies in $\Omega_{2}^{\text {sig }}$ and for $3 \pi / 4 \leq \theta \leq 5 \pi / 4$, it is the steepest descent curve. The reason why we choose a part of the circle as the contour for the remaining angles is to ensure the uniform boundedness of the Cauchy operators (see Section 5 of [3].) This does not affect the asymptotics since as we will see the main contribution to the asymptotics comes from neighborhood of $z=-1$.

Define the regions $\Omega_{j}^{(3)}, j=1, \cdots, 4$ as in Figure 4 . Define $m^{(3)}(z ; k ; t)$ by, as in $(5.9)$ of [3],

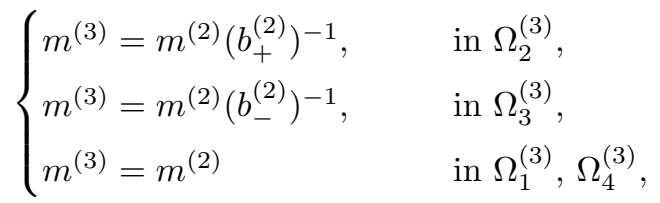




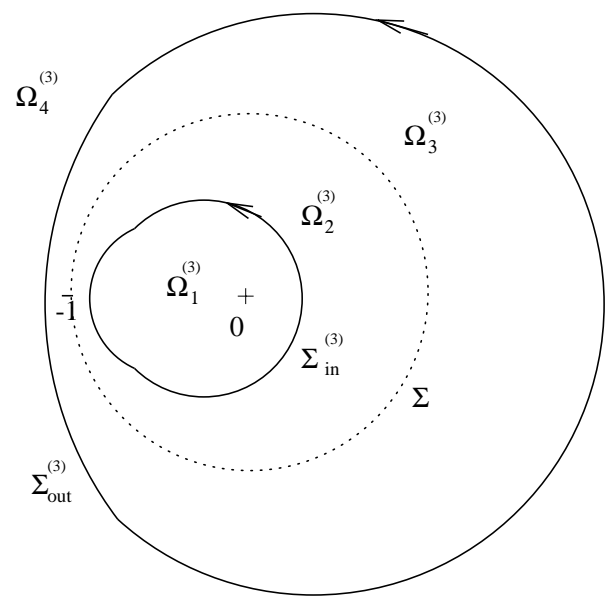

Figure 4: $\Sigma^{(3)}$ and $\Omega^{(3)}$ when $\gamma<1$

where $b_{ \pm}^{(2)}$ are defined in 10.6). Then $m^{(3)}$ solves a new RHP with the jump matrix $v^{(3)}(z ; k ; t)$ where

$$
\begin{cases}v^{(3)}=\left(\begin{array}{cc}
1 & -(-1)^{k} z^{k} e^{t\left(z-z^{-1}\right)} \\
0 & 1
\end{array}\right) & \text { on } \quad \Sigma_{\text {in }}^{(3)} \\
v^{(3)}=\left(\begin{array}{cc}
1 & 0 \\
(-1)^{k} z^{-k} e^{-t\left(z-z^{-1}\right)} & 1
\end{array}\right) & \text { on } \quad \Sigma_{\text {out }}^{(3)} .\end{cases}
$$

Set $w^{(3)}:=v^{(3)}-I$. For $z \in \Sigma_{i n}^{(3)}$, from the choice of $\Sigma^{(3)}$ and $(10.32)$, the (12)-entry of the jump matrix satisfies for $3 \pi / 4 \leq \arg z \leq 5 \pi / 4$,

$$
\left|z^{k} e^{t\left(z-z^{-1}\right)}\right|=e^{k F\left(\rho_{\theta}, \theta ; \frac{2 t}{k}\right)} \leq e^{-\frac{2 \sqrt{2}}{3} k\left(1+\frac{2 t}{k} \cos \theta\right)^{3 / 2}} \leq e^{-\frac{2 \sqrt{2}}{3} k\left(1-\frac{2 t}{k}\right)^{3 / 2}} \leq e^{-\frac{2}{3} M^{3 / 2}},
$$

and for $0 \leq \arg z \leq 3 \pi / 4$ or $5 \pi / 4 \leq \arg z<2 \pi$,

$$
\left|z^{k} e^{t\left(z-z^{-1}\right)}\right|=e^{k F\left(\rho_{3 \pi / 4}, \theta ; \frac{2 t}{k}\right)} \leq e^{k F\left(\rho_{3 \pi / 4}, \frac{3 \pi}{4} ; \frac{2 t}{k}\right)} \leq e^{-\frac{2 \sqrt{2}}{3} k\left(1+\frac{2 t}{k} \cos \frac{3 \pi}{4}\right)^{3 / 2}} \leq e^{-\frac{1}{24} k} .
$$

From (10.12), similar estimates hold for $z^{-k} e^{-t\left(z-z^{-1}\right)}, z \in \Sigma_{\text {out }}^{(3)}$. Thus we have

$$
\left\|w^{(3)}\right\|_{L^{\infty}\left(\Sigma^{(3)}\right)} \leq C e^{-\frac{2 \sqrt{2}}{3} k\left(1-\frac{2 t}{k}\right)^{3 / 2}} .
$$

Also there exists $M_{0}$ such that for $M>M_{0},\left\|C_{w^{(3)}}\right\|_{L^{2}\left(\Sigma^{(3)}\right) \rightarrow L^{2}\left(\Sigma^{(3)}\right)} \leq c_{1}<1$, and hence (10.15) holds. This is precisely (5.18) of [3]. For this derivation, we do not need the extension (10.32) of (5.13) in [3]. But for the improved $L^{1}$ norm estimate of $w^{(3)}$, which we do now, we need (10.32).

Note that $|d z| \leq C|d \theta|$ on $\Sigma^{(3)}$. Using the estimates in (10.36) and (10.37),

$$
\int_{\Sigma_{i n}^{(3)}}\left|z^{k} e^{t\left(z-z^{-1}\right)}\right||d z| \leq C \int_{\frac{3 \pi}{4}}^{\frac{5 \pi}{4}} e^{-\frac{2 \sqrt{2}}{3} k\left(1+\frac{2 t}{k} \cos \theta\right)^{3 / 2}} d \theta+C \int_{[0,2 \pi) \backslash[3 \pi / 4,5 \pi / 4]} e^{-\frac{1}{24} k} d \theta .
$$


The second integral is clearly less than $C e^{-\frac{1}{24} k}$. For the first integral, recall the inequality $(x+y)^{a} \geq x^{a}+y^{a}$, $x, y>0, a \geq 1$. Then using the inequality $1+\cos \theta \geq \frac{1}{2 \sqrt{2}}(\theta-\pi)^{2}$ for $\theta \in\left[\frac{3 \pi}{4}, \frac{5 \pi}{4}\right]$, together with the condition $a k \leq 2 t$, the first integral is less than or equal to

$$
\int_{\frac{3 \pi}{4}}^{\frac{5 \pi}{4}} e^{-\frac{2 \sqrt{2}}{3} k\left[\left(1-\frac{2 t}{k}\right)^{3 / 2}+\left(\frac{2 t}{k}(1+\cos \theta)\right)^{3 / 2}\right]} d \theta \leq e^{-\frac{2 \sqrt{2}}{3} k\left(1-\frac{2 t}{k}\right)^{3 / 2}} \int_{\frac{3 \pi}{4}}^{\frac{5 \pi}{4}} e^{-\frac{a^{3 / 2}}{3 \cdot 2^{3 / 4} k|\theta-\pi|^{3}}} d \theta
$$

where last inequality is less than or equal to $C k^{-1 / 3}$ for some constant $C>0$. Therefore adjusting constants, we obtain

$$
\int_{\Sigma_{i n}^{(3)}}\left|z^{k} e^{t\left(z-z^{-1}\right)}\right||d z| \leq \frac{C}{k^{1 / 3}} e^{-\frac{2 \sqrt{2}}{3} k\left(1-\frac{2 t}{k}\right)^{3 / 2}}
$$

We have similar estimates on $\Sigma_{\text {out }}^{(3)}$. Therefore,

$$
\left\|w^{(3)}\right\|_{L^{1}\left(\Sigma^{(3)}\right)} \leq \frac{C}{k^{1 / 3}} e^{-\frac{2 \sqrt{2}}{3} k\left(1-\frac{2 t}{k}\right)^{3 / 2}} .
$$

This is a refinement of (5.23) in [3].

Now from (10.15), we have

$$
m^{(3)}(z)=I+\frac{1}{2 \pi i} \int_{\Sigma^{(3)}} \frac{w^{(3)}(s)}{s-z} d s+\frac{1}{2 \pi i} \int_{\Sigma^{(3)}} \frac{\left[\left(I-C_{w^{(3)}}\right)^{-1} C_{w^{(3)}} I\right](s) w^{(3)}(s)}{s-z} d s, \quad z \notin \Sigma^{(3)} .
$$

In [3], only the term $m_{22}^{(3)}(z)$ (at $z=0$ ) was of interest. But then $w_{22}^{(3)}=0$, and the first integral in (10.43) was zero. As computed in (5.20) of [3], the second integral is bounded by the product of the $L^{\infty}$ and $L^{1}$ norms of $w^{(3)}$, and then due to (10.38), the estimate $\left\|w^{(3)}\right\|_{L^{1}} \leq C k^{-1 / 3}$ in (5.23) of [3] was enough to control $m_{22}^{(3)}$. But in the present paper, we need estimates of $m_{12}^{(3)}$ for $\pi_{k}(z)$, and hence we need an estimate of the first integral which is same as a bound on the $L^{1}$ norm of $w^{(3)}$. The $L^{1}$ bound (5.23) obtained in [3] is not good enough for this purpose, and we need an improved estimate on the $L^{1}$ norm of $w^{(3)}$. Now by (10.42), the first integral is less than or equal to

$$
\frac{C}{\operatorname{dist}\left(z, \Sigma^{(3)}\right) k^{1 / 3}} e^{-\frac{2 \sqrt{2}}{3} k\left(1-\frac{2 t}{k}\right)^{3 / 2}}
$$

while, as in (5.20) of [3], the second integral is less than or equal to, by using (10.38) and (10.42),

$$
\begin{aligned}
& \frac{1}{2 \pi \operatorname{dist}\left(z, \Sigma^{(3)}\right)}\left\|\left(I-C_{w^{(3)}}\right)^{-1} C_{w^{(3)}} I\right\|_{L^{2}}\left\|w^{(3)}\right\|_{L^{2}} \\
& \leq \frac{1}{2 \pi \operatorname{dist}\left(z, \Sigma^{(3)}\right)}\left\|\left(I-C_{w^{(3)}}\right)^{-1}\right\|_{L^{2} \rightarrow L^{2}}\left\|C_{w^{(3)}} I\right\|_{L^{2}}\left\|w^{(3)}\right\|_{L^{2}} \\
& \leq \frac{C}{\operatorname{dist}\left(z, \Sigma^{(3)}\right)}\left\|w^{(3)}\right\|_{L^{2}}^{2} \\
& \leq \frac{C}{\operatorname{dist}\left(z, \Sigma^{(3)}\right)}\left\|w^{(3)}\right\|_{L^{\infty}}\left\|w^{(3)}\right\|_{L^{1}} \\
& \leq \frac{C}{\operatorname{dist}\left(z, \Sigma^{(3)}\right) k^{1 / 3}} e^{-\frac{4 \sqrt{2}}{3} k\left(1-\frac{2 t}{k}\right)^{3 / 2}} .
\end{aligned}
$$


When $z=0$, $\operatorname{dist}\left(z, \Sigma^{(3)}\right) \geq c_{1}>0$, hence using (10.34), 10.7), (10.8) and (5.5), (5.6), we obtain Proposition 5.1 (ii).

As in (10.17)-(10.19), from (10.13), (10.7), (10.9) and (5.6), we have

$$
\begin{array}{ll}
\pi_{k}(z ; t)=-(-1)^{k} e^{-t z} m_{12}^{(3)}(z ; k ; t), & z \in \Omega_{1}^{(3)}, \\
\pi_{k}(z ; t)=z^{k} e^{-t z^{-1}} m_{11}^{(3)}(z ; k ; t), & z \in \Omega_{4}^{(3)}, \\
\pi_{k}(z ; t)=z^{k} e^{-t z^{-1}} m_{11}^{(3)}(z ; k ; t)-(-1)^{k} e^{-t z} m_{12}^{(3)}(z ; k ; t), & z \in \Omega_{2}^{(3)} \cup \Omega_{3}^{(3)} .
\end{array}
$$

Define $x$ by

$$
\frac{2 t}{k}=1-\frac{x}{2^{1 / 3} k^{2 / 3}}
$$

as in Proposition 5.6. Let $0<b<1$ be a fixed number. Given $b$, from the beginning, we could have chosen $0<a<1$ such that

$$
\rho_{\theta_{b}}=\frac{1-\sqrt{1-a^{2} \cos ^{2} \theta_{b}}}{-a \cos \theta_{b}}
$$

is strictly greater than $b$ for some $\pi / 2 \leq \theta_{b}<\pi$. Note that in (10.33) defining $\Sigma^{(3)}$, the choice of $3 \pi / 4$ and $5 \pi / 4$ was arbitrary. Instead of $3 \pi / 4$ and $5 \pi / 4$, this time we use $\theta_{b}$ and $2 \pi-\theta_{b}$, and carry this forward through the later calculations. Thus we obtain the same estimates of $(10.44)$ and $(10.45)$ with different constants $C$. Now $|z| \leq b$ lies in $\Omega_{1}^{(3)}$ and $|z| \geq b^{-1}$ lies in $\Omega_{4}^{(3)}$. Since the distance $\operatorname{dist}\left(z, \Sigma^{(3)}\right) \geq c_{2}>0$, using (10.43), (10.44), (10.45), (10.46) and (10.47), we obtain (5.29) and (5.30) in Proposition 5.6 for $M \leq x \leq(1-a) 2^{1 / 3} k^{2 / 3}$. Since in (10.20) and (10.21) in Subsubsection 10.1.1, the choice of $0<a<1$ was arbitrary, we obtain (5.29) and (5.30) in Proposition 5.6 for all $x \geq M$.

On the other hand, let $0<L<2^{-3 / 2} \sqrt{M}$ be a fixed number. Set $\alpha=1-2^{4 / 3} k^{-1 / 3} w$ with $-L \leq w \leq L$ as in Proposition 5.6. From the inequality $\frac{1-\sqrt{1-\gamma^{2}}}{\gamma} \leq 1-\sqrt{1-\gamma}$ for all $0 \leq \gamma \leq 1$, we have

$$
\rho_{\pi}=\frac{1-\sqrt{1-\left(\frac{2 t}{k}\right)^{2}}}{\frac{2 t}{k}} \leq 1-\sqrt{1-\frac{2 t}{k}} \leq 1-\frac{\sqrt{M}}{2^{1 / 6} k^{1 / 3}} .
$$

But

$$
\alpha=1-\frac{2^{4 / 3} w}{k^{1 / 3}} \geq 1-\frac{2^{4 / 3} L}{k^{1 / 3}} .
$$

Hence $\operatorname{dist}\left(-\alpha, \Sigma^{(3)}\right) \geq C k^{-1 / 3}$. Thus from (10.43), (10.44) and (10.45), we have

$$
\left|m^{(3)}(-\alpha ; k ; t)-I\right| \leq C e^{-\frac{2}{3} x^{3 / 2}},
$$

which together with (10.48) (note that $-\alpha \in \Omega_{2}^{(3)} \cup \Omega_{3}^{(3)}$ ), implies that

$$
\begin{aligned}
\left|e^{-t \alpha} \pi_{k}(-\alpha ; t)\right| & \leq\left|(-\alpha)^{k} e^{-t\left(\alpha-\alpha^{-1}\right)}\right|\left(1+C e^{-c|x|^{3 / 2}}\right)+C e^{-c|x|^{3 / 2}}, \\
\left|e^{-t \alpha^{-1}}(-\alpha)^{-k} \pi_{k}(-\alpha ; t)-1\right| & \leq C e^{-c|x|^{3 / 2}}+\left|(-\alpha)^{-k} e^{t\left(\alpha-\alpha^{-1}\right)}\right| C e^{-c|x|^{3 / 2}} .
\end{aligned}
$$


For large $k, \alpha \geq \frac{1}{2}$ for all $-L \leq w \leq L$, hence using (10.26), we obtain as in (10.27) and (10.28),

$$
\left|(-\alpha)^{k} e^{-t\left(\alpha-\alpha^{-1}\right)}\right|=e^{k F\left(-\alpha ; \frac{2 t}{k}\right)} \leq e^{-2 w x+\frac{32}{2}|w|^{3}} \leq C e^{c|x|},
$$

and

$$
\left|(-\alpha)^{-k} e^{t\left(\alpha-\alpha^{-1}\right)}\right|=e^{k F\left(-\alpha^{-1} ; \frac{2 t}{k}\right)} \leq C e^{c|x|}
$$

Thus from (10.54) and (10.55), we obtain (5.31), (5.32) in Proposition 5.6.

10.1.3 The case $k-M 2^{-1 / 3} k^{1 / 3} \leq 2 t \leq k$ for some $M>0$.

In this case, as $k \rightarrow \infty$ the point $z=-\rho_{\pi}$ on the deformed contour $\Sigma^{(3)}$ defined in (10.33) approaches $z=-1$ rapidly, and so we need to pay special attention to the neighborhood of $z=-1$. More precisely, we need to introduce the so-called parametrix for the RHP around $z=-1$, which is an approximate local solution.

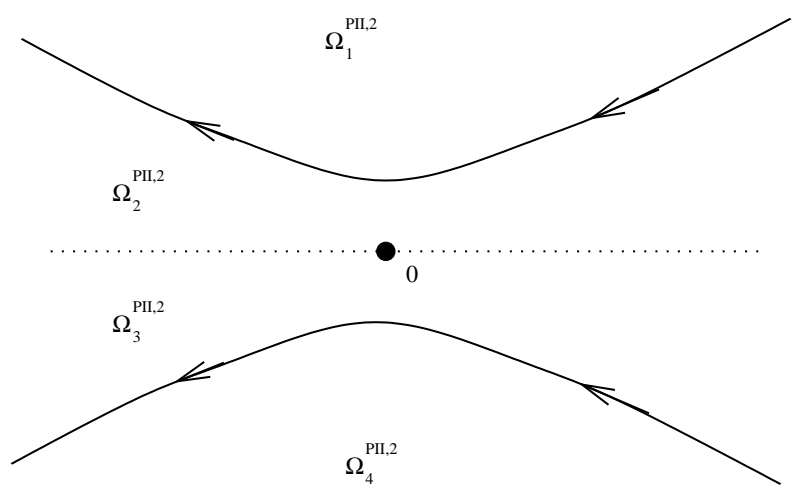

Figure 5: $\Sigma^{P I I, 2}$ and $\Omega_{j}^{P I I, 2}$

Recall the RHP (2.15) for the Painlevé II equation. Let $\Sigma^{P I I, 2}=\Sigma_{1}^{P I I, 2} \cup \Sigma_{2}^{P I I, 2}$ be a contour of the general shape indicated in Figure 5 . Asymptotically for large $z$, the curves are straight lines of angle less than $\pi / 3$. See the paragraph after (2.18) in [3] for more precise discussions on the curve. We will define the exact shape of $\Sigma^{P I I, 2}$ below. Define $m^{P I I, 2}(z ; x)$ by

$$
\begin{cases}m^{P I I, 2}(z, x)=m(z ; x)\left(\begin{array}{cc}
1 & e^{-2 i\left(\frac{4}{3} z^{3}+x z\right)} \\
0 & 1
\end{array}\right) & \text { in } \Omega_{2}^{P I I, 2}, \\
m^{P I I, 2}(z, x)=m(z ; x)\left(\begin{array}{ccc}
1 & 0 \\
e^{2 i\left(\frac{4}{3} z^{3}+x z\right)} & 1
\end{array}\right) & \text { in } \Omega_{3}^{P I I, 2}, \\
m^{P I I, 2}(z, x)=m(z ; x) & \text { in } \Omega_{1}^{P I I, 2}, \Omega_{4}^{P I I, 2},\end{cases}
$$

where $m(z ; x)$ is the solution of the RHP for the PII equation given in (2.15). Then $m^{P I I, 2}$ solves a new RHP 
(see $(2.19)$ of [3])

$$
\left\{\begin{array}{l}
m^{P I I, 2} \quad \text { is analytic in } \mathbb{C} \backslash \Sigma^{P I I, 2}, \\
m_{+}^{P I I, 2}=m_{-}^{P I I, 2}\left(\begin{array}{cc}
1 & -e^{-2 i\left(\frac{4}{3} z^{3}+x z\right)} \\
0 & 1
\end{array}\right) \quad \text { in } \Sigma_{1}^{P I I, 2}, \\
m_{+}^{P I I, 2}=m_{-}^{P I I, 2}\left(\begin{array}{cr}
1 & 0 \\
e^{2 i\left(\frac{4}{3} z^{3}+x z\right)} & 1
\end{array}\right) \quad \text { in } \Sigma_{2}^{P I I, 2}, \\
m^{P I I, 2}=I+O\left(\frac{1}{z}\right) \quad \text { as } z \rightarrow \infty .
\end{array}\right.
$$

Also $m_{1}^{P I I, 2}(x)$ defined by $m^{P I I, 2}(z ; x)=I+\frac{m_{1}^{P I I, 2}(x)}{z}+O\left(z^{-2}\right)$ satisfies

$$
m_{1}^{P I I, 2}(x)=m_{1}(x),
$$

where $m_{1}(x)$ is defined in a similar manner in (2.16).

Set $x$ by

$$
\frac{2 t}{k}=1-\frac{x}{2^{1 / 3} k^{2 / 3}}
$$

We define $\Sigma^{(3)}$ and $m^{(3)}$ as in (10.33) and (10.34). Now we proceed as in (5.25)-(5.35) of [3]. Let $\mathcal{O}$ be the ball of radius $\epsilon$ around $z=-1$, where $\epsilon>0$ is a small fixed number. Define the map (see the equation displayed between (5.26) and (5.27) of [3)

$$
\lambda(z):=-i 2^{-4 / 3} k^{1 / 3} \frac{1}{2}\left(z-z^{-1}\right)
$$

in $\mathcal{O}$. Define $\Sigma^{P I I, 2}$ by $\Sigma^{P I I, 2} \cap \lambda(\mathcal{O}):=\lambda\left(\Sigma^{(3)} \cap \mathcal{O}\right)$ and extend it smoothly outside $\lambda(\mathcal{O})$ as indicated in (5.29) of [3]. And define $m^{P I I, 2}$ as above using this contour. Now we define the parametrix by (see the equation displayed between (5.30) and (5.31) of [3])

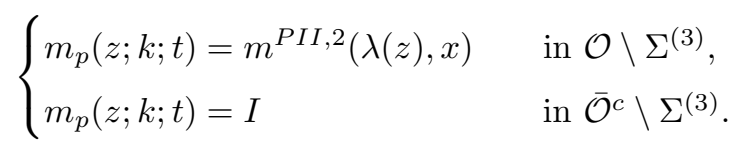

It is proved in (5.25)-(5.34) in [3] that if we take $\epsilon$ small enough but fix it, then the ratio

$$
R(z ; k ; t):=m^{(3)} m_{p}^{-1}
$$

solves a new RHP

$$
\left\{\begin{array}{l}
R(z ; k ; t) \quad \text { is analytic in } \mathbb{C} \backslash \Sigma_{R}, \\
R_{+}(z ; k ; t)=R_{-}(z ; k ; t) v_{R}(z ; k ; t), \quad \text { on } \Sigma_{R}, \\
R(z ; k ; t)=I+O\left(\frac{1}{z}\right), \quad \text { as } z \rightarrow \infty,
\end{array}\right.
$$


where $\Sigma_{R}:=\partial \mathcal{O} \cup \Sigma^{(3)}$, and the jump matrix satisfies (see (5.34) of [3])

$$
\begin{cases}\left\|v_{R}-I\right\|_{L^{\infty}} \leq \frac{C}{k^{2 / 3}} & \text { on } \mathcal{O} \cap \Sigma^{(3)}, \\ \left\|v_{R}-I\right\|_{L^{\infty}} \leq C e^{-c k} & \text { on } \mathcal{O}^{c} \cap \Sigma^{(3)}, \\ \left\|v_{R}-I+\frac{m_{1}^{P I I, 2}(x)}{\lambda(z)}\right\|_{L^{\infty}} \leq \frac{C}{k^{2 / 3}} & \text { on } \partial \mathcal{O}, \text { as } k \rightarrow \infty,\end{cases}
$$

with some positive constants $C$ and $c$ which may depend on $M$. Set $w_{R}:=v_{R}-I$. Using (10.15) which holds generally, we have (see (5.35) and the preceding calculations in [3])

$$
\begin{aligned}
R(z ; k ; t) & =I+\frac{1}{2 \pi i} \int_{\Sigma_{R}} \frac{\left(\left(I-C_{w_{R}}\right)^{-1} I\right)(s)\left(w_{R}(s)\right)}{s-z} d s \\
& =I+\frac{1}{2 \pi i} \int_{\Sigma_{R}} \frac{v_{R}(s)-I}{s-z} d s+\frac{1}{2 \pi i} \int_{\Sigma_{R}} \frac{\left[\left(I-C_{w_{R}}\right)^{-1} C_{w_{R}} I\right](s) w_{R}(s)}{s-z} d s .
\end{aligned}
$$

Now the absolute value of the second integral is less than or equal to (recall that $|\lambda(z)|=O\left(k^{-1 / 3}\right)$ for $z \in \partial \mathcal{O}$ )

$$
\begin{aligned}
& \frac{C}{\operatorname{dist}\left(z, \Sigma_{R}\right)}\left\|\left(I-C_{w_{R}}\right)^{-1}\right\|_{L^{2}\left(\Sigma_{R}\right) \rightarrow L^{2}\left(\Sigma_{R}\right)}\left\|C_{w_{R}} I\right\|_{L^{2}\left(\Sigma_{R}\right)}\left\|w_{R}\right\|_{L^{2}\left(\Sigma_{R}\right)} \\
& \leq \frac{C}{\operatorname{dist}\left(z, \Sigma_{R}\right)}\left\|w_{R}\right\|_{L^{2}\left(\Sigma_{R}\right)}^{2} \\
& \leq \frac{\epsilon C}{\operatorname{dist}\left(z, \Sigma_{R}\right) k^{2 / 3}},
\end{aligned}
$$

and similarly, the first integral satisfies

$$
\left|\frac{1}{2 \pi i} \int_{\Sigma_{R}} \frac{v_{R}(s)-I}{s-z} d s+\frac{m_{1}^{P I I, 2}(x)}{2 \pi i} \int_{\partial \mathcal{O}} \frac{1}{\lambda(s)(s-z)} d s\right| \leq \frac{\epsilon C}{\operatorname{dist}\left(z, \Sigma_{R}\right) k^{2 / 3}} .
$$

Hence

$$
\left|m^{(3)}(z ; k ; t)\left(m_{p}(z ; k ; t)\right)^{-1}-I+\frac{m_{1}^{P I I, 2}(x)}{2 \pi i} \int_{\partial \mathcal{O}} \frac{1}{\lambda(s)(s-z)} d s\right| \leq \frac{\epsilon C}{\operatorname{dist}\left(z, \Sigma_{R}\right) k^{2 / 3}} .
$$

This is an extension of (5.35) to the case when $z \neq 0$.

For $z=0$, from (10.63) and (10.64), $R(0)=m^{(3)}(0)$, and $\operatorname{dist}\left(0, \Sigma_{R}\right) \geq c_{1}>0$. Note that $\lambda(s)$ is analytic in $\mathcal{O}$ except at $s=-1$, and

$$
\lambda(s)=-i 2^{-4 / 3} k^{1 / 3}\left[(s+1)+\frac{1}{2}(s+1)^{2}+\cdots\right], \quad s \sim-1 .
$$

By a residue calculation for (10.70), we have, as in (5.35) of [3])

$$
m^{(3)}(0 ; k ; t)=I+\frac{i 2^{4 / 3} m_{1}^{P I I, 2}(x)}{k^{1 / 3}}+O\left(\frac{1}{k^{2 / 3}}\right) .
$$

Thus using (2.17) and (2.18), from (5.5), (5.6), 10.7), (10.8) and (10.34), we obtain Proposition 5.1 (iii) of the case when $0 \leq x \leq M$.

We now prove Proposition 5.2 when $x \geq 0$. Since the choice of $M$ was arbitrary in our calculations, for fixed $x$ we choose $M>0$ large enough so that $x<M$. Let $z \in \mathbb{C} \backslash \Sigma$ be fixed. We first assume $|z|<1$. 
By modifying the contour $\Sigma^{(3)}$, if necessary, as in (10.50) and the following paragraph, we have $z \in \Omega_{1}^{(3)}$ and $\operatorname{dist}\left(z, \Sigma^{(3)}\right) \geq c_{1}>0$. Thus from (10.70), $|R(z)-I| \leq C k^{-1 / 3}$ with some constant $C$ which depends on $x$. Thus from (5.6), (10.7), (10.34) and (10.64), we obtain the first limit of (5.13). Similar calculation applies to the case $|z|>1$, and we obtain the first limit of (5.14). The second limits of (5.13) and (5.14) follow from the first limits of (5.14) and (5.13) respectively by replacing $z \rightarrow 1 / z$. Hence this extends the calculation (5.35) of [3] to the case when $z$ is bounded away from the contour.

Finally, we prove Proposition 5.4 when $x>0$. Set

$$
\alpha=1-\frac{2^{4 / 3} w}{k^{1 / 3}}, \quad w \text { fixed, }
$$

and

$$
\frac{2 t}{k}=1-\frac{x}{2^{1 / 3} k^{2 / 3}}, \quad x>0 \text { fixed. }
$$

In this case, $-\alpha \in \mathcal{O}$. By a residue calculation again, for $w$ not equal to 0 ,

$$
\begin{aligned}
\frac{1}{2 \pi i} \int_{\partial \mathcal{O}} \frac{1}{\lambda(s)(s+\alpha)} d s & =\frac{i 2^{4 / 3}}{(-1+\alpha) k^{1 / 3}}+\frac{1}{\lambda(-\alpha)} \\
& =-\frac{i}{w}+\frac{i}{w+\frac{2^{1 / 3}}{k^{1 / 3}} w^{2}+\cdots}=O\left(\frac{1}{k^{1 / 3}}\right) .
\end{aligned}
$$

When $w=0$, we have the same order $O\left(k^{-1 / 3}\right)$ by a similar calculation. On the other hand, since

$$
\rho_{\pi}=\frac{1-\sqrt{1-\left(\frac{2 t}{k}\right)^{2}}}{\frac{2 t}{k}}=1-\frac{2^{1 / 3} \sqrt{x}}{k^{1 / 3}}+\frac{x}{2^{1 / 3} k^{2 / 3}}+O\left(\frac{1}{k}\right),
$$

using (10.73) we have $\operatorname{dist}\left(-\alpha, \Sigma_{R}\right) \geq C k^{-2 / 3}$. Thus we obtain from (10.70)

$$
|R(-\alpha ; k ; t)-I| \leq \epsilon C
$$

Using $\lambda(-\alpha) \sim-i w$, from (10.63) and (10.64), we have

$$
\lim _{k \rightarrow \infty} m^{(3)}(-\alpha ; k ; t)=m^{P I I, 2}(-i w, x)
$$

since $\epsilon$ is arbitrarily small. On the other hand, from the conditions on $t$ and $\alpha$, we have

$$
\lim _{k \rightarrow \infty} \alpha^{k} e^{-t\left(\alpha-\alpha^{-1}\right)}=e^{\frac{8}{3} w^{3}-2 x w}
$$

Thus using (10.34), we obtain

$$
\begin{aligned}
& \lim _{k \rightarrow \infty} m^{(2)}(-\alpha ; k ; t)=m^{P I I, 2}(-i w, x), \quad-\alpha \in \Omega_{1}^{(3)}, \Omega_{4}^{(3)}, \\
& \lim _{k \rightarrow \infty} m^{(2)}(-\alpha ; k ; t)=m^{P I I, 2}(-i w, x)\left(\begin{array}{cc}
1 & -e^{\frac{8}{3} w^{3}-2 x w} \\
0 & 1
\end{array}\right), \quad-\alpha \in \Omega_{2}^{(3)}, \\
& \lim _{k \rightarrow \infty} m^{(2)}(-\alpha ; k ; t)=m^{P I I, 2}(-i w, x)\left(\begin{array}{cc}
1 & 0 \\
-e^{-\frac{8}{3} w^{3}+2 x w} & 1
\end{array}\right), \quad-\alpha \in \Omega_{3}^{(3)} .
\end{aligned}
$$


Now finally using (10.58), for each fixed $w$ and $x$, we have

$$
\lim _{k \rightarrow \infty} m^{(2)}(-\alpha ; k ; t)=m(-i w ; x) .
$$

From 10.7)-(10.10) and (10.79), this implies Proposition 5.4 of the case when $x>0$. This was a new computation we had to do in the present paper in order to include the case when $\alpha \rightarrow 1$.

\subsection{When $(2 t) / k>1$.}

Throughout this subsection, we set

$$
\gamma:=\frac{2 t}{k}>1
$$

We need some definitions from [3]. Set $0<\theta_{c}<\pi$ by $\sin ^{2} \frac{\theta_{c}}{2}=\frac{1}{\gamma}$. Define a probability measure on an arc (see (4.13) of [3]),

$$
d \mu(\theta):=\frac{\gamma}{\pi} \cos \left(\frac{\theta}{2}\right) \sqrt{\frac{1}{\gamma}-\sin ^{2}\left(\frac{\theta}{2}\right)} d \theta, \quad-\theta_{c} \leq \theta \leq \theta_{c},
$$

and define a constant (see (4.14) of [3])

$$
l:=-\gamma+\log \gamma+1
$$

Now we introduce the so-called $g$-function (see (4.8) of [3]),

$$
g(z ; k ; t):=\int_{-\theta_{c}}^{\theta_{c}} \log \left(z-e^{i \theta}\right) d \mu(\theta), \quad z \in \mathbb{C} \backslash \Sigma \cup(-\infty,-1] .
$$

The measure $d \mu(\theta)$ is the equilibrium measure of a certain variational problem and the constant $l$ is a related constant (see Section 4 of [3]). For each $|\theta| \leq \theta_{c}$, the branch is chosen such that $\log \left(z-e^{i \theta}\right)$ is analytic in $\mathbb{C} \backslash(-\infty,-1] \cup\left\{e^{i \phi}:-\pi \leq \phi \leq \theta\right\}$ and behaves like $\log z$ as $z \in \mathbb{R} \rightarrow+\infty$. The basic properties of $g(z)$ are summarized in Lemma 4.2 of [3]. In general, the role of the $g$-function in RHP analysis, first introduced in [19] and then generalized in [17, is to replace exponentially growing terms in the jump matrix by oscillating or exponentially decaying terms. In [13], the authors introduced a $g$-function of a form similar to (10.87) to analyze an RHP associated to orthogonal polynomials on the real line. The above $g$-function (10.87) introduced in [3] is an adaptation of their work to the circle case. When $0 \leq \gamma \leq 1$, the related equilibrium measure is (see (4.12) of [3])

$$
d \mu(\theta)=\frac{1}{2 \pi}(1+\gamma \cos \theta) d \theta, \quad-\pi \leq \theta<\pi,
$$

with the related constant $l=0$, and hence (see (4.15) of [3])

$$
g(z)= \begin{cases}\log z-\frac{\gamma}{2 z}, & |z|>1, z \notin(-\infty,-1) \\ -\frac{\gamma}{2} z+\pi i, & |z|<1 .\end{cases}
$$

Since $g(z)$ is explicit in this case, we did not introduce it of the form (10.87) in the previous subsection. 
Then up to (10.104, we follows the procedure in Section 6 of [3]. Write $\Sigma=C_{1} \cup \overline{C_{2}}$ where $C_{2}:=\left\{e^{i \theta}\right.$ : $\left.-\theta_{c}<\theta<\theta_{c}\right\}$ and $C_{1}:=\Sigma \backslash \overline{C_{2}}$. Define $m^{(1)}(z ; k ; t)$ by

$$
m^{(1)}(z ; k ; t):=e^{\frac{k l}{2} \sigma_{3}} Y(z ; k ; t) e^{-k g(z ; k ; t) \sigma_{3}} e^{-\frac{k l}{2} \sigma_{3}},
$$

where $\sigma_{3}=\left(\begin{array}{cc}1 & 0 \\ 0 & -1\end{array}\right)$. Then $m^{(1)}$ solves (see (6.1) in [3]) a new RHP

$$
\left\{\begin{array}{l}
m^{(1)}(z ; k ; t) \quad \text { is analytic in } \mathbb{C} \backslash \Sigma, \\
m_{+}^{(1)}(z ; k ; t)=m_{-}^{(1)}(z ; k ; t)\left(\begin{array}{cr}
e^{-2 k \widetilde{\alpha}(z ; k ; t)} & (-1)^{k} \\
0 & e^{2 k \widetilde{\alpha}(z ; k ; t)}
\end{array}\right) \text { on } C_{2}, \\
m_{+}^{(1)}(z ; k ; t)=m_{-}^{(1)}(z ; k ; t)\left(\begin{array}{cc}
1 & (-1)^{k} e^{-2 k \widetilde{\alpha}-(z ; k ; t)} \\
0 & 1
\end{array}\right) \text { on } C_{1}, \\
m^{(1)}(z ; k ; t)=I+O\left(\frac{1}{z}\right) \quad \text { as } \quad z \rightarrow \infty
\end{array}\right.
$$

where $\widetilde{\alpha}(z ; k ; t)$ is defined by (see Lemma 6.1 in [3])

$$
\widetilde{\alpha}(z ; k ; t):=-\frac{\gamma}{4} \int_{e^{i \theta_{c}}}^{z} \frac{s+1}{s^{2}} \sqrt{\left(s-e^{i \theta_{c}}\right)\left(s-e^{-i \theta_{c}}\right)} d s, \quad \xi:=e^{i \theta_{c}} .
$$

(Notation: We use $\widetilde{\alpha}$ here instead of $\alpha$ in [3] to avoid confusion with $\alpha$ in (10.140) below.) The branch is chosen such that $\sqrt{\left(s-e^{i \theta_{c}}\right)\left(s-e^{-i \theta_{c}}\right)}$ is analytic in $\mathbb{C} \backslash \overline{C_{1}}$ and behaves like $s$ as $s \in \mathbb{R} \rightarrow+\infty$.

Define $m^{(2)}(z ; k ; t)$ as in (10.3). Then $m^{(2)}$ solves a new RHP, normalized as $z \rightarrow \infty$, with the jump matrices $((6.2)$ of [3] $)$

$$
\begin{cases}v^{(2)}(z ; k ; t)=\left(\begin{array}{cc}
1 & -e^{-2 k \widetilde{\alpha}(z ; k ; t)} \\
e^{2 k \widetilde{\alpha}(z ; k ; t)} & 0
\end{array}\right) & \text { on } C_{2}, \\
v^{(2)}(z ; k ; t)=\left(\begin{array}{cc}
e^{-2 k \widetilde{\alpha}-(z ; k ; t)} & -1 \\
1 & 0
\end{array}\right) & \text { on } C_{1} .\end{cases}
$$

Through the changes $Y \rightarrow m^{(1)} \rightarrow m^{(2)}$, we have

$$
\begin{array}{rlrl}
Y_{11}(z ; k ; t) & =-e^{k g(z ; k ; t)} m_{12}^{(2)}(z ; k ; t), & & |z|<1, \\
Y_{21}(z ; k ; t) & =-(-1)^{k} e^{k g(z ; k ; t)+k l} m_{22}^{(2)}(z ; k ; t), & & |z|<1, \\
Y_{11}(z ; k ; t) & =e^{k g(z ; k ; t)} m_{11}^{(2)}(z ; k ; t), & & |z|>1, \\
Y_{21}(z ; k ; t) & =(-1)^{k} e^{k g(z ; k ; t)+k l} m_{21}^{(2)}(z ; k ; t), & |z|>1 .
\end{array}
$$

Set $\Sigma^{(3)}:=\overline{C_{1}} \cup \widetilde{C}_{\text {inside }} \cup \widetilde{C}_{\text {outside }}$ as in the Figure 6 , which divides $\mathbb{C}$ into four regions, $\Omega_{j}^{(3)}, j=1, \cdots, 4$. Again, there is a certain freedom choosing the shape of $\widetilde{C}_{\text {inside }}$ and $\widetilde{C}_{\text {outside. For }}$. Foxample, $\widetilde{C}_{\text {inside }}$ (resp., $\widetilde{C}_{\text {outside }}$ ) can be any smooth curve lying in $\Omega_{2}^{(3)}$ (resp. $\Omega_{3}^{(3)}$ ) connecting $e^{i \theta_{c}}$ and $e^{-i \theta_{c}}$; the precise requirement 


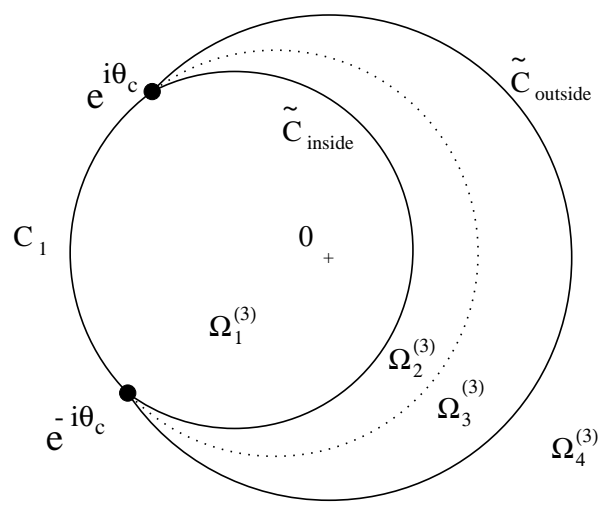

Figure 6: $\Sigma^{(3)}$ and $\Omega^{(3)}$ when $\gamma>1$

is given in [3] (see also (10.100)-(10.102 below). Define $m^{(3)}(z ; k ; t)$ by (see p.1151 of [3])

$$
\begin{cases}m^{(3)}=m^{(2)}\left(\begin{array}{cc}
1 & -e^{-2 k \widetilde{\alpha}(z ; k ; t)} \\
0 & 1
\end{array}\right)^{-1} & \text { in } \Omega_{2}^{(3)}, \\
m^{(3)}=m^{(2)}\left(\begin{array}{cc}
1 & 0 \\
e^{2 k \widetilde{\alpha}(z ; k ; t)} & 1
\end{array}\right) & \text { in } \Omega_{3}^{(3)}, \\
m^{(3)}=m^{(2)} & \text { in } \Omega_{1}^{(3)}, \Omega_{4}^{(3)} .\end{cases}
$$

Then $m^{(3)}$ solves a RHP, normalized as $z \rightarrow \infty$, with the jump matrix given by

$$
v^{(3)}(z ; k ; t)= \begin{cases}\left(\begin{array}{cc}
1 & -e^{-2 k \widetilde{\alpha}(z ; k ; t)} \\
0 & 1
\end{array}\right) & \text { on } \widetilde{C}_{\text {inside }}, \\
\left(\begin{array}{cc}
1 & 0 \\
e^{2 k \widetilde{\alpha}(z ; k ; t)} & 1
\end{array}\right) & \text { on } \widetilde{C}_{\text {outside }}, \\
\left(\begin{array}{cc}
e^{-2 k \widetilde{\alpha}-(z ; k ; t)} & -1 \\
1 & 0
\end{array}\right) & \text { on } C_{1} .\end{cases}
$$

From the properties of $g(z)$, it is proved in [3] (between (6.4) and (6.4)) that

$$
\begin{gathered}
e^{-k \widetilde{\alpha}-(z ; k ; t)} \rightarrow 0 \quad \text { as } \quad k \rightarrow \infty, \quad z \in C_{1}, \\
e^{-k \widetilde{\alpha}(z ; k ; t)} \rightarrow 0 \quad \text { as } \quad k \rightarrow \infty, \quad z \in \widetilde{C}_{\text {inside }}, \\
e^{k \widetilde{\alpha}(z ; k ; t)} \rightarrow 0 \quad \text { as } \quad k \rightarrow \infty, \quad z \in \widetilde{C}_{\text {outside }} .
\end{gathered}
$$

The choice of $\widetilde{C}_{\text {inside }}$ and $\widetilde{C}_{\text {outside }}$ is precisely for these properties. Here the convergence is uniform for any compact part of the each contour away from the end points $e^{i \theta_{c}}$ and $e^{-i \theta_{c}}$, but is not uniform on the whole contour. This gives rise the technical difficulty which will be overcome below using the idea of parametrix. 
Formally $v^{(3)} \rightarrow v^{\infty}$ as $k \rightarrow \infty$ where

$$
\begin{cases}v^{\infty}(z)=\left(\begin{array}{ll}
1 & 0 \\
0 & 1
\end{array}\right) & \text { on } \widetilde{C}_{\text {inside }} \cup \widetilde{C}_{\text {outside }} \\
v^{\infty}(z)=\left(\begin{array}{cc}
0 & -1 \\
1 & 0
\end{array}\right) & \text { on } C_{1} .\end{cases}
$$

Thus we expect that $m^{(3)}$ converges to $m^{\infty}$, the solution of the RHP $m_{+}^{\infty}=m_{-}^{\infty} v^{\infty}$ with $m^{\infty} \rightarrow I$ as $z \rightarrow \infty$. The solution $m^{\infty}$ is easily given by (see Lemma 6.2 in [3])

$$
m^{\infty}(z)=\left(\begin{array}{cc}
\frac{1}{2}\left(\beta+\beta^{-1}\right) & \frac{1}{2 i}\left(\beta-\beta^{-1}\right) \\
-\frac{1}{2 i}\left(\beta-\beta^{-1}\right) & \frac{1}{2}\left(\beta+\beta^{-1}\right)
\end{array}\right),
$$

where $\beta(z):=\left(\frac{z-e^{i \theta_{c}}}{z-e^{-i \theta_{c}}}\right)^{1 / 4}$, which is analytic $\mathbb{C} \backslash \bar{C}_{1}$ and $\beta \sim+1$ as $z \in \mathbb{R} \rightarrow+\infty$

\subsubsection{The case $2 t \geq a k$ for some $a>1$.}

The parametrix $m_{p}(z)$ is introduced in (6.25)-(6.31) in [3], and has the following properties. In the neighborhood $\mathcal{O}$ of size $\epsilon$ around the points $e^{i \theta_{c}}$ and $e^{-i \theta_{c}}, m_{p}(z)$ is constructed using the Airy function in such a way that $\left(m_{p}(z)\right)_{+}=\left(m_{p}(z)\right)_{-} v^{(3)}(z)$ for $z \in \Sigma^{(3)} \cap \mathcal{O}$, and $\left\|m_{p}\left(m^{\infty}\right)^{-1}-I\right\|_{L^{\infty}(\partial \mathcal{O})}=O\left(k^{-1}\right)$. In $\mathbb{C} \backslash \mathcal{O}$, we set $m_{p}(z):=m^{\infty}(z)$. Then the ratio $R(z):=m^{(3)}(z) m_{p}^{-1}(z)$ has no jump on $\Sigma^{(3)} \cap \mathcal{O}$, and has a jump $v_{R}:=w_{R}+I$ converging to $I$ uniformly of order $O\left(k^{-1}\right)$ on $\partial \mathcal{O}$, and of order $O\left(e^{-c k}\right)$ on $\Sigma^{(3)} \cap \mathcal{O}^{c}$ as $k \rightarrow \infty$. This implies that $R(z)=I+O\left(k^{-1}\right)$ for any $z \in \mathbb{C} \backslash \Sigma_{p}, \Sigma_{p}:=\left(\Sigma^{(3)} \cap \mathcal{O}^{c}\right) \cup \partial \mathcal{O}$. Moreover following the arguments in Section 8 of [14], the error is uniform up to the boundary in each open regions in $\mathbb{C} \backslash \Sigma_{p}$. In particular, for

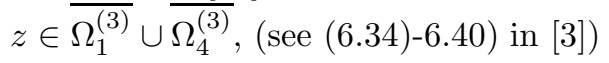

$$
m^{(3)}(z)=\left(I+O\left(k^{-1}\right)\right) m^{\infty}(z) .
$$

Here the error is uniform for $a k \leq 2 t \leq b k$ for some $0<a<b$. For the case $(2 t) / k \rightarrow \infty$, by shrinking the size of $\mathcal{O}$ properly, we again obtain uniform error (see [2]). Therefore for any $a>0$, we obtain uniformity in (10.105) for $a k \leq 2 t$.

When $z=0, \beta(0)=-i e^{i \theta_{c} / 2}$ and $g(0)=\pi i$ (Lemma 4.2 (vi) of [3]), Also (10.98) says $m^{(3)}(0)=m^{(2)}(0)$. Thus Proposition 5.1 (v) follows from (10.94) and (10.95), as in (6.4) of [3].

Now we consider Proposition 5.6 of the case where $x \leq-2^{1 / 3}(a-1) k^{2 / 3}$. For $z=-\alpha$ real, $|\beta(-\alpha)|=1$, so $m^{\infty}(-\alpha)$ is bounded. Hence from (10.98) and (10.94), (10.96), we have for $\alpha \geq 1$,

$$
\left|\pi_{k}(-\alpha ; k ; t)\right| \leq C\left|e^{k g(-\alpha ; k ; t)}\right| .
$$

Then we proceed as in 10.109)-(10.121) of the following subsubsection to obtain the proper estimate. 
10.2.2 The case $k+M 2^{-1 / 3} k^{1 / 3} \leq 2 t \leq a k$ for some $a>1$ and $M>M_{0}$.

In this case, the points $e^{i \theta_{c}}$ and $e^{-i \theta_{c}}$ are allowed to approach -1 , but the rate is restricted:

$$
\left|e^{i \theta_{c}}+1\right|=2\left(1-\frac{k}{2 t}\right)^{1 / 2} \geq \frac{\sqrt{M}}{k^{1 / 3}}
$$

for $k$ large. We now take the neighborhood $\mathcal{O}$ to be of size $\epsilon \sqrt{\frac{2 t}{k}-1}$ around $e^{i \theta_{c}}$ and $e^{-i \theta_{c}}$. From $(10.107), \mathcal{O}$ consists of two disjoint disks and their boundaries do not touch the real axis. We introduce the same parametrix $m_{p}$ as in the previous subsubsection. Then we have a similar result: there is $M_{0}>0$ such that for $M>M_{0}$,

$$
m^{(3)}(z)=\left(I+O\left(\frac{1}{k\left(\frac{2 t}{k}-1\right)}\right)\right) m^{\infty}(z)
$$

for $z \in \overline{\Omega_{1}^{(3)}} \cup \overline{\Omega_{4}^{(3)}}$; this is proved in $(6.34)-(6.40)$ of $[3]$.

When $z=0$, as in the previous subsubsection, we obtain Proposition 5.1 (iv), as in [3].

Now we prove Proposition 5.6 when $x \leq-M$. As in the previous subsubsection, we have

$$
\left|\pi_{k}(-\alpha ; k ; t)\right| \leq C\left|e^{k g(-\alpha ; k ; t)}\right|
$$

Now we need an estimate of $g(-\alpha ; k ; t)$; as we mentioned at the beginning of Section 10 , this is the second way in which we must extend [3]. Note that

$$
\begin{aligned}
\operatorname{Re}(g(-\alpha)) & =\frac{1}{2} \int_{-\theta_{c}}^{\theta_{c}} \log \left(1+\alpha^{2}+2 \alpha \cos \theta\right) d \mu(\theta) \\
& =\log 2+\frac{1}{2} \log \frac{\alpha}{\gamma}+I(s)
\end{aligned}
$$

where

$$
I(s)=\frac{1}{\pi} \int_{-1}^{1} \log \left(s^{2}-x^{2}\right) \sqrt{1-x^{2}} d x, \quad s:=\frac{\sqrt{\gamma}(1+\alpha)}{2 \sqrt{\alpha}}>1 .
$$

The inequality $s>1$ follows from the arithmetic-geometric mean inequality and the assumption $\gamma>1$. A residue calculation gives us

$$
I^{\prime}(y)=\frac{1}{\pi} \int_{-1}^{1} \frac{2 y}{y^{2}-x^{2}} \sqrt{1-x^{2}} d x=2 y-2 \sqrt{y^{2}-1}, \quad y>1 .
$$

Integrating from 1 to $s>1$, we have

$$
I(s)=s^{2}-1-2 \int_{1}^{s} \sqrt{y^{2}-1} d y+I(1) .
$$

The constant $I(1)$ can be evaluated (cf. Lemma 4.3 (ii)-(a) in 3 ):

$$
I(1)=\frac{1}{\pi} \int_{-\frac{\pi}{2}}^{\frac{\pi}{2}} \log \left(\sin ^{2} \theta\right) \sin ^{2} \theta d \theta=\frac{1}{2}-\log 2 .
$$


Thus we have

$$
\operatorname{Re}(g(-\alpha))=-\frac{1}{2}+\frac{1}{2} \log \frac{\alpha}{\gamma}+s^{2}-s \sqrt{s^{2}-1}+\log \left(s+\sqrt{s^{2}-1}\right) .
$$

Assume $0<\alpha \leq 1$. We change the variables $\gamma, \alpha$ into $s, \xi$ where $s$ is defined in (10.111) and

$$
\xi:=\left(\frac{\gamma}{\alpha}\right)^{1 / 2}>1
$$

Then

$$
F(\xi):=g(-\alpha)-\frac{\gamma}{2} \alpha=-\frac{1}{2}-\log \xi-\frac{1}{2} \xi^{2}+2 s \xi-s^{2}-s \sqrt{s^{2}-1}+\log \left(s+\sqrt{s^{2}-1}\right) .
$$

Differentiating with respect to $\xi$, we find

$$
F^{\prime}(\xi)=-\frac{1}{\xi}-\xi+2 s
$$

Thus the maximum of $F$ occurs at $\xi=s+\sqrt{s^{2}-1}$. But $F\left(s+\sqrt{s^{2}-1}\right)=0$, hence $F(\xi) \leq 0$. Thus we obtain

$$
\left|e^{-t \alpha} \pi_{k}(-\alpha ; k)\right| \leq C e^{k \operatorname{Re}\left(g(-\alpha ; k ; t)-\frac{\gamma}{2} \alpha\right)} \leq C, \quad 0<\alpha \leq 1 .
$$

For $\alpha \geq 1$, note that

$$
\operatorname{Re}(g(-\alpha))=\log \alpha+\operatorname{Re}\left(g\left(-\alpha^{-1}\right)\right)
$$

Thus using (10.119), we have

$$
\left|e^{-t \alpha^{-1}}(-\alpha)^{-k} \pi_{k}(-\alpha ; k)\right| \leq C e^{k \operatorname{Re}\left(g(-\alpha ; k ; t)-\frac{\gamma}{2} \alpha^{-1}-\log \alpha\right)}=C e^{k \operatorname{Re}\left(g\left(-\alpha^{-1} ; k ; t\right)-\frac{\gamma}{2} \alpha^{-1}\right)} \leq C .
$$

10.2.3 Case $k<2 t \leq k+M 2^{-1 / 3} k^{1 / 3}$ for some $M>0$.

First we introduce $m^{P I I, 3}$ as in (2.22)-(2.28) of [3]. Set

$$
g^{P I I}(z):=\frac{4}{3}\left(z^{2}+\frac{x}{2}\right)^{3 / 2},
$$

which is analytic in $\mathbb{C} \backslash\left[-\sqrt{\frac{-x}{2}}, \sqrt{\frac{-x}{2}}\right]$ and behaves like $\frac{4}{3} z^{3}+x z+\frac{x^{2}}{8 z}+O\left(z^{-3}\right)=: \theta_{P I I}(z)+O\left(z^{-1}\right)$ as $z \rightarrow+\infty$. Let $\Sigma^{P I I, 3}:=\cup_{j=1}^{5} \Sigma_{j}^{P I I, 3}$ as shown in Figure 7 . The angles of the rays with the real line are between 0 and $\pi / 3$. Recall that $m(z ; x)$ solves $(2.15)$ the RHP for PII equation. Define $m^{P I I, 3}(z ; x)$ by

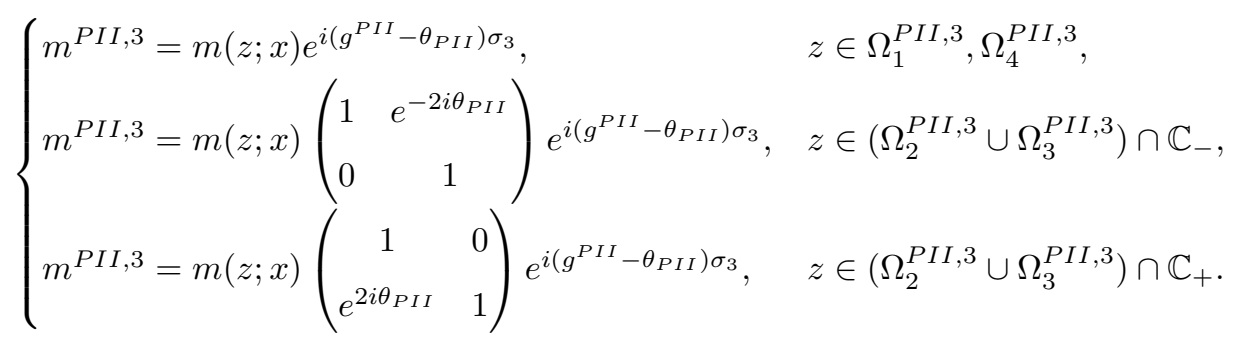




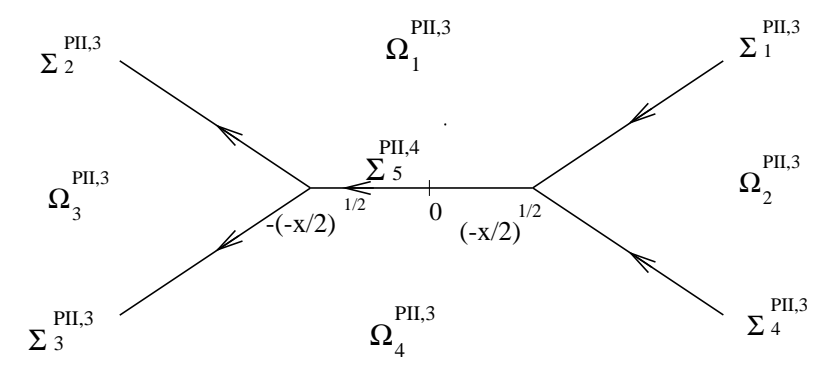

Figure 7: $\Sigma^{P I I, 3}$ and $\Omega_{j}^{P I I, 3}$

Then $m^{(3)}$ solves the RHP (see (2.25) in [3]) normalized at $\infty$ with the jump matrix

$$
v^{(3)}(z ; k ; t)= \begin{cases}\left(\begin{array}{cc}
1 & 0 \\
e^{2 i g^{P I I}} & 1
\end{array}\right) & \text { on } \Sigma_{1}^{P I I, 3}, \Sigma_{2}^{P I I, 3} \\
\left(\begin{array}{cc}
1 & -e^{-2 i g^{P I I}} \\
0 & 1
\end{array}\right) & \text { on } \Sigma_{3}^{P I I, 3}, \Sigma_{4}^{P I I, 3} \\
\left(\begin{array}{cc}
e^{-2 i g_{-}^{P I I}} & -1 \\
1 & 0
\end{array}\right) & \text { on } \Sigma_{5}^{P I I, 3} .\end{cases}
$$

Also we have

$$
m_{1}(x)=m_{1}^{P I I, 3}(x)-\left(\frac{i x^{2}}{8}\right) \sigma_{3}
$$

where $m^{P I I, 3}(z ; x)=I+\frac{m_{1}^{P I I, 3}(x)}{z}+O\left(z^{-2}\right)$ as $z \rightarrow \infty$.

Now as before, set $x$ by

$$
\frac{2 t}{k}=1-\frac{x}{2^{1 / 3} k^{2 / 3}}
$$

Hence we have $-M \leq x<0$ in this subsubsection. We now proceed as in case (iii) of Section 6 in $[3]$. Define the parametrix

$$
\begin{cases}m_{p}(z ; k ; t)=m^{P I I, 3}(\lambda(z), x) & \text { in } \quad \mathcal{O} \backslash \Sigma^{(3)}, \\ m_{p}(z ; k ; t)=I & \text { in } \quad \overline{\mathcal{O}}^{c} \backslash \Sigma^{(3)},\end{cases}
$$

where $\lambda(z)$ is defined in (10.62) and $\mathcal{O}$ is a small neighborhood of size $\epsilon>0$ around $z=-1$ (see [3] case (iii) of Section 6 for details). As in Subsubsection 10.1.3, the ratio $R(z ; k ; t):=m^{(3)} m_{p}^{-1}$ satisfies a new RHP, normalized at $\infty$, with jump matrix $v_{R}$ satsifying the estimate (10.66) where $m_{1}^{P I I, 2}(x)$ is replaced by $m_{1}^{P I I, 3}(x)$. Hence we have

$$
\left|m^{(3)}(z ; k ; t)\left(m_{p}(z ; k ; t)\right)^{-1}-I+\frac{m_{1}^{P I I, 3}(x)}{2 \pi i} \int_{\partial \mathcal{O}} \frac{1}{\lambda(s)(s-z)} d s\right| \leq \frac{\epsilon C}{\operatorname{dist}\left(z, \Sigma_{R}\right) k^{2 / 3}},
$$

which is hidden in the derivation of (6.19) of [3]. 
Then as in (10.72), we have

$$
m^{(3)}(0 ; k ; t)=I+\frac{i 2^{4 / 3} m_{1}^{P I I, 3}(x)}{k^{1 / 3}}+O\left(\frac{1}{k^{2 / 3}}\right),
$$

which is a (direct) extension of (6.19) in [3]. Now from (10.98) and (10.125) (see (6.19) of [3]),

$$
m^{(2)}(0 ; k ; t)=I+\frac{i 2^{4 / 3} m_{1}(x)-2^{-5 / 3} x^{2} \sigma_{3}}{k^{1 / 3}}+O\left(\frac{1}{k^{2 / 3}}\right) .
$$

Hence using $e^{k l}=1-\frac{x^{2}}{2^{5 / 3} k^{1 / 3}}+O\left(k^{-2 / 3}\right)$ and $g(0)=\pi i$, 10.94) and (10.95) yield Proposition 5.1 (iii) in the case $-M \leq x<0$, as in [3].

For the proof of Proposition 5.2, note that, as before, for each fixed $z \in \mathbb{C} \backslash \Sigma$, we can use the freedom of the shape of $\Sigma^{(3)}$ (and $\Sigma_{R}$ ) so that $z \in \Omega_{1}^{(3)} \cup \Omega_{4}^{(3)}$, and $\operatorname{dist}\left(z, \Sigma_{R}\right) \geq c_{1}>0$. Thus we obtain

$$
\lim _{k \rightarrow \infty} m^{(2)}(z ; k ; t)=I, \quad z \in \mathbb{C} \backslash \Sigma \text { fixed. }
$$

From (10.94) and (10.96), we have

$$
\begin{array}{llrl}
\lim _{k \rightarrow \infty} e^{-k g(z ; k ; t)} \pi_{k}(z) & =0, & & |z|<1, \\
\lim _{k \rightarrow \infty} e^{-k g(z ; k ; t)} \pi_{k}(z) & =1, & & |z|>1 .
\end{array}
$$

This is an extension of the calculation of [3] where $z=0$ is given. Now in order to prove Proposition 5.2, we need further analysis which is an extension in the second category as mentioned as the beginning of Section 10 . Since $\gamma=(2 t) / k$, for the proof of Proposition 5.2, it is enough to show that

$$
\begin{aligned}
\lim _{k \rightarrow \infty}(-1)^{k} e^{k\left[g(z ; k ; t)+\frac{\gamma}{2} z\right]} & =1, & & |z|<1, \\
\lim _{k \rightarrow \infty} e^{k\left[g(z ; k ; t)+\frac{\gamma}{2} z^{-1}-\log z\right]} & =1, & & |z|>1 .
\end{aligned}
$$

But the proof of Lemma 4.3 (ii) of [3] says that for $|z|>1, z \notin(-\infty,-1)$,

$$
g(z)=\frac{1}{2} \log z-\frac{\gamma}{4}\left(z+z^{-1}\right)+\frac{\gamma}{2}+\frac{\gamma}{4} \int_{1_{+0}}^{z} \frac{s+1}{s^{2}} \sqrt{\left(s-e^{i \theta_{c}}\right)\left(s-e^{-i \theta_{c}}\right)} d s+g_{-}(1),
$$

where the integral is taken over a curve from $1_{+0}$ to $z$ lying in $\{z \in \mathbb{C}:|z|>1, z \notin(-\infty,-1)\}$. Here $\sqrt{\left(s-e^{i \theta_{c}}\right)\left(s-e^{-i \theta_{c}}\right)}$ is analytic in $\mathbb{C} \backslash C_{1}$ and behaves like $s$ as $s \in \mathbb{R} \rightarrow+\infty$, and $\log z$ is analytic in $\mathbb{C} \backslash(-\infty, 0]$ and is real for $z \mathbb{R}_{+}$. Calculations in the same proof, together with Lemma 4.2 (viii) of [3], give us $g_{-}(1)=-\frac{1}{2}-\frac{1}{2} \log \gamma$. Also using $\sin ^{2} \frac{\theta_{c}}{2}=\frac{1}{\gamma}$, for $|s|>1, s \notin(-\infty,-1)$,

$$
\sqrt{\left(s-e^{i \theta_{c}}\right)\left(s-e^{-i \theta_{c}}\right)}=(s+1)-\frac{2 s}{s+1}(\gamma-1)+O\left((\gamma-1)^{2}\right) .
$$

Thus expanding in $\gamma-1$, we have

$$
g(z)+\frac{\gamma}{2} z^{-1}-\log z=O\left((\gamma-1)^{2}\right)=O\left(k^{-4 / 3}\right),
$$


which implies (10.135). Similar calculations using for $|z|<1, z \notin(-1,0]$,

$$
g(z)=\frac{1}{2} \log z-\frac{\gamma}{4}\left(z+z^{-1}\right)+\frac{\gamma}{2}+\frac{\gamma}{4} \int_{1_{+0}}^{z} \frac{s+1}{s^{2}} \sqrt{\left(s-e^{i \theta_{c}}\right)\left(s-e^{-i \theta_{c}}\right)} d s+g_{+}(1),
$$

and $g_{+}(1)=-\frac{1}{2}-\frac{1}{2} \log z+\pi i$ yield (10.134).

For the proof of Proposition 5.4 when $x<0$, set

$$
\alpha=1-\frac{2^{4 / 3} w}{k^{1 / 3}} .
$$

In this case, we need a new argument as $\alpha \rightarrow 1$. When $w$ and $x$ are fixed, again as in (10.77), we have $\lim _{k \rightarrow \infty} R(-\alpha ; k ; t)=I$, which implies that

$$
\lim _{k \rightarrow \infty} m^{(3)}(-\alpha ; k ; t)=m^{P I I, 3}(-i w, x) .
$$

From (6.8) of [3], we have

$$
\lim _{k \rightarrow \infty} k \widetilde{\alpha}(-\alpha ; k ; t)=i \frac{4}{3}\left((-i w)^{2}+\frac{x}{2}\right)^{3 / 2}=i g^{P I I}(-i w),
$$

which from (10.98) and 10.123) implies

$$
\lim _{k \rightarrow \infty} m^{(2)}(-\alpha ; k ; t)=m(-i w, x) e^{i\left(g^{P I I}(-i w)-\theta_{P I I}(-i w)\right) \sigma_{3}} .
$$

Now we compute the large $k$ limit of $k g(-\alpha ; k ; t)-t \alpha$ when $w>0$, and of $k g(-\alpha ; k ; t)-t \alpha^{-1}-\log \alpha$ when $w<0$. For $-\pi<\theta<\pi, \lim _{\epsilon \downarrow 0} \arg \left(-\alpha+i \epsilon-e^{i \theta}\right)=\pi+\tan ^{-1}\left(\frac{\sin \theta}{\alpha+\cos \theta}\right)$ where $-\pi<\tan ^{-1} \phi<\pi$. Since $\tan ^{-1}\left(\frac{\sin \theta}{\alpha+\cos \theta}\right)$ is odd in $\theta$, we have from 10.115),

$$
\begin{aligned}
\lim _{\epsilon \downarrow 0} g(-\alpha+i \epsilon) & =\lim _{\epsilon \downarrow 0} \operatorname{Re} g(-\alpha+i \epsilon)+\pi i \\
& =-\frac{1}{2}+\frac{1}{2} \log \frac{\alpha}{\gamma}+s^{2}-s \sqrt{s^{2}-1}+\log \left(s+\sqrt{s^{2}-1}\right)+\pi i
\end{aligned}
$$

where $s=\frac{\sqrt{\gamma}(1+\alpha)}{2 \sqrt{\alpha}}>1$. Under the stated conditions on $\gamma$ and $\alpha$, as $k \rightarrow \infty$,

$$
\begin{aligned}
\frac{\sqrt{\gamma}(1+\alpha)}{2 \sqrt{\alpha}} & =1+\left(\frac{w^{2}}{2^{1 / 3}}-\frac{x}{2^{4 / 3}}\right) \cdot \frac{1}{k^{2 / 3}}+\frac{2 w^{3}}{k}+O\left(\frac{1}{k^{-4 / 3}}\right), \\
\frac{\alpha}{\gamma} & =1-\frac{2^{4 / 3} w}{k^{1 / 3}}+\frac{x}{2^{1 / 3} k^{2 / 3}}-\frac{2 x w}{k}+O\left(\frac{1}{k^{-4 / 3}}\right) .
\end{aligned}
$$

Note that $\lim _{\epsilon \downarrow 0} g(-\alpha+i \epsilon)-\lim _{\epsilon \downarrow 0} g(-\alpha-i \epsilon)$ is $2 \pi i$ for $\alpha>1$, and is 0 for $0<\alpha<1$. Therefore we obtain

$$
\begin{aligned}
\lim _{k \rightarrow \infty}(-1)^{k} e^{k g(-\alpha)-t \alpha} & =e^{\frac{4}{3} w^{3}-x w-\frac{4}{3}\left(w^{2}-\frac{x}{2}\right)^{3 / 2}}, \quad w>0, \\
\lim _{k \rightarrow \infty}(-1)^{k} e^{k g(-\alpha)-t \alpha^{-1}-k \log \alpha} & =e^{-\frac{4}{3} w^{3}+x w-\frac{4}{3}\left(w^{2}-\frac{x}{2}\right)^{3 / 2}}, \quad w<0 .
\end{aligned}
$$

Also being careful of branch, we have

$$
\begin{array}{ll}
i\left(g^{P I I}(-i w)-\theta_{P I I}(-i w)\right)=\frac{4}{3} w^{3}-x w-\frac{4}{3}\left(w^{2}-\frac{x}{2}\right)^{3 / 2}, \quad w>0, \\
i\left(g^{P I I}(-i w)-\theta_{P I I}(-i w)\right)=\frac{4}{3} w^{3}-x w+\frac{4}{3}\left(w^{2}-\frac{x}{2}\right)^{3 / 2}, \quad w<0 .
\end{array}
$$

Since $\lim _{k \rightarrow \infty} e^{k l}=1$, using (10.94)-110.97) and 10.143), this implies (5.21)-(5.24) when $x<0$. 


\subsection{Proof of Proposition 5.8}

The analysis in this section is new, and is needed for the proof of Proposition 5.8 . Let $\alpha>1$ be fixed. Set

$$
\frac{t}{k}=\frac{\alpha}{\alpha^{2}+1}-\frac{\alpha\left(\alpha^{2}-1\right)^{1 / 2}}{\left(\alpha^{2}+1\right)^{3 / 2}} \cdot \frac{x}{\sqrt{k}}, \quad x \in \mathbb{R} \backslash\{0\} \text { is fixed. }
$$

We are interested in the asymptotics of $e^{-\alpha t}(-\alpha)^{l} \pi_{k}\left(-\alpha^{-1} ; t\right)$. Since $\frac{2 \alpha}{\alpha^{2}+1}<1$ and $x$ is fixed, we are in the case of Subsubsection 10.1.1 and/or 10.1.2. We define $m^{(1)}$ and $m^{(2)}$ as in (10.1) and (10.3). Recall that we have certain freedom in the choice of $\Sigma^{(3)}$. We choose a contour passing through the saddle points of

$$
f(z):=\frac{t}{k}\left(z-z^{-1}\right)+\log (-z)
$$

the exponent of the (12) entry of $v^{(2)}$ divided by $k$. The saddle points (see (10.31) and the following discussion) are $-\rho_{\pi}$ and $-\rho_{\pi}^{-1}$, where

$$
\rho_{\pi}=\frac{1-\sqrt{1-(2 t / k)^{2}}}{2 t / k}=\frac{1}{\alpha}-\frac{\left(\alpha^{2}+1\right)^{1 / 2}}{\alpha\left(\alpha^{2}-1\right)^{1 / 2}} \cdot \frac{x}{\sqrt{k}}+O\left(\frac{1}{k}\right)=: \rho_{c}+O\left(\frac{1}{k}\right) .
$$

Take $\delta>0$ and $\epsilon>0$ small such that $\Sigma_{c}:=\left\{-\rho_{c}+i s:-k^{\delta-1 / 2} \leq s \leq \epsilon k^{\delta-1 / 2}\right\}$ lies inside the open unit disc for all $k \geq 1$. Define (see Figure 8) $\Sigma^{(3)}:=\Sigma_{\text {in }}^{(3)} \cup \Sigma_{\text {out }}^{(3)}$ by $\Sigma_{\text {in }}^{(3)}:=\Sigma_{c} \cup \Sigma_{r}$ and $\Sigma_{\text {out }}^{(3)}:=\left\{r^{-1} e^{i \phi}: r e^{i \phi} \in \Sigma_{\text {in }}^{(3)}\right\}$,

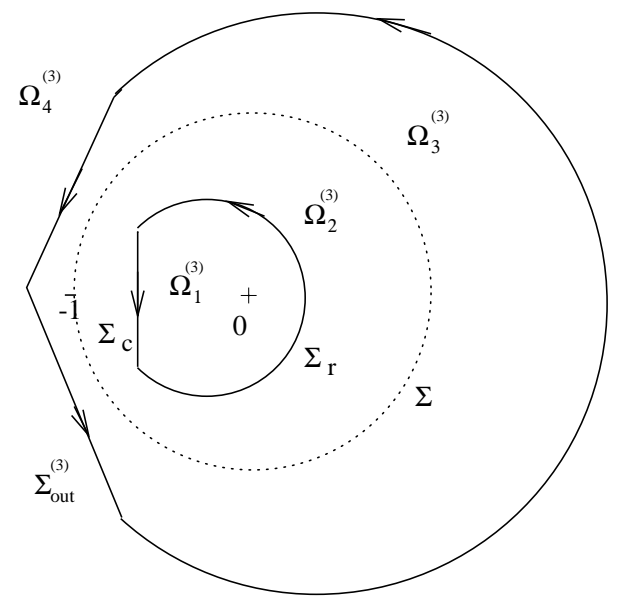

Figure 8: $\Sigma^{(3)}$ and $\Omega^{(3)}$

where $\Sigma_{r}:=\left\{\left|-\rho_{c}+i \epsilon k^{\delta-1 / 2}\right| e^{i \theta}:|\theta|<\theta_{0}\right\},-\rho_{c}+i \epsilon k^{\delta-1 / 2}=\left|-\rho_{c}+i \epsilon k^{\delta-1 / 2}\right| e^{i \theta_{0}}$. Let $\Omega_{j}^{(3)}$ be as in Figure 8 and define $m^{(3)}$ as in (10.34). As in (10.46)-(10.48), the quantities we are interested in are

$$
\begin{aligned}
e^{-\alpha t}(-\alpha)^{k} \pi_{k}\left(-\alpha^{-1} ; t\right) & =-\alpha^{k} e^{-t\left(\alpha-\alpha^{-1}\right)} m_{12}^{(3)}\left(-\alpha^{-1} ; k ; t\right), & x<0, \\
e^{-\alpha t}(-\alpha)^{k} \pi_{k}\left(-\alpha^{-1} ; t\right) & =-\alpha^{k} e^{-t\left(\alpha-\alpha^{-1}\right)} m_{12}^{(3)}\left(-\alpha^{-1} ; k ; t\right)+m_{11}^{(3)}\left(-\alpha^{-1} ; k ; t\right), & x>0 .
\end{aligned}
$$

For the estimates of $w^{(3)}:=v^{(3)}-I$, note that for any fixed $0<\rho<1, \operatorname{Re} f\left(\rho e^{i \theta}\right)=F\left(\rho e^{i \theta} ; \frac{2 t}{k}\right)$ (recall (10.11) ) is increasing in $0<\theta<\pi$, and is decreasing in $\pi<\theta<2 \pi$, hence $\left\|e^{k f(z)}\right\|_{L^{\infty}\left(\Sigma_{r}\right)}=e^{k \operatorname{Re} f\left(-\rho_{c}+\epsilon k^{\delta-1 / 2}\right)}$. 
But we have

$$
f\left(-\rho_{c}+i a\right)=\frac{t}{k}\left(\alpha-\alpha^{-1}\right)-\log \alpha-\frac{1}{2}\left(\frac{\alpha^{2}\left(\alpha^{2}-1\right)}{\alpha^{2}+1} a^{2}+\frac{x^{2}}{k}\right)+O\left(k^{-\frac{3}{2}+2 \delta}\right), \quad|a| \leq \epsilon k^{\delta-1 / 2} .
$$

Thus,

$$
\left\|\alpha^{k} e^{-t\left(\alpha-\alpha^{-1}\right)} e^{k f(z)}\right\|_{L^{\infty}\left(\Sigma_{r}\right)} \leq C e^{-c k^{2 \delta}}
$$

and also using

$$
\alpha^{-k} e^{t\left(\alpha-\alpha^{-1}\right)}=e^{k\left[\log \alpha-\frac{\alpha^{2}-1}{\alpha^{2}+1}+O\left(\frac{1}{\sqrt{k}}\right)\right]}, \quad \log \alpha-\frac{\alpha^{2}-1}{\alpha^{2}+1}>0, \text { for } \alpha>1,
$$

we have

$$
\left\|e^{k f(z)}\right\|_{L^{\infty}\left(\Sigma_{r}\right)} \leq C e^{-c k} .
$$

On the other hand, one can directly check that $\operatorname{Re} f\left(-\rho_{c}+a\right)$ has its maximum at $a=0$ for $-\epsilon k^{\delta-1 / 2} \leq a \leq$ $\epsilon k^{\delta-1 / 2}$, hence $\left\|e^{k f(z)}\right\|_{L^{\infty}\left(\Sigma_{c}\right)}=e^{k \operatorname{Re} f\left(-\rho_{c}\right)}$. Again 10.156) and 10.158) yield

$$
\left\|e^{k f(z)}\right\|_{L^{\infty}\left(\Sigma_{c}\right)} \leq e^{-c k} .
$$

Similarly, we have $\left\|e^{-k f(z)}\right\|_{L^{\infty}\left(\Sigma_{\text {out }}^{(3)}\right)} \leq e^{-c k}$. Now calculations as in Subsubsection 10.1.2 give us the result (10.43). Hence using (10.158) and (10.160) and noting $\operatorname{dist}\left(-\alpha^{-1}, \Sigma^{(3)}\right)=\frac{\left(\alpha^{2}+1\right)^{1 / 2}}{\alpha\left(\alpha^{2}-1\right)^{1 / 2}} \cdot \frac{x}{\sqrt{k}}$, we have

$$
\begin{aligned}
m_{11}^{(3)}\left(-\alpha^{-1} ; k ; t\right) & =1+O\left(\sqrt{k} e^{-2\left(1-\epsilon_{1}\right) c_{1} k}\right) \\
\alpha^{k} e^{-t\left(\alpha-\alpha^{-1}\right)} m_{12}^{(3)}\left(-\alpha^{-1} ; k ; t\right) & =\alpha^{k} e^{-t\left(\alpha-\alpha^{-1}\right)} \int_{\Sigma_{c}} \frac{(-s)^{k} e^{t\left(s-s^{-1}\right)}}{s+\alpha^{-1}} \frac{d s}{2 \pi i}+O\left(\sqrt{k} e^{-c k^{2 \delta}}\right) .
\end{aligned}
$$

To evaluate the integral asymptotically, first we change the variable by $s=-\rho_{c}-\frac{i\left(\alpha^{2}+1\right)^{1 / 2}}{\alpha\left(\alpha^{2}-1\right)^{1 / 2}} \cdot \frac{y}{\sqrt{k}}$. Then from (10.156), the numerator of the integrand becomes

$$
\alpha^{-k} e^{t\left(\alpha-\alpha^{-1}\right)} e^{-\frac{1}{2}\left(y^{2}+x^{2}\right)+O\left(k^{-\frac{1}{2}+2 \delta}\right)} .
$$

Hence setting $A:=\frac{\epsilon \alpha\left(\alpha^{2}-1\right)^{1 / 2}}{\left(\alpha^{2}+1\right)^{1 / 2}}$,

$$
\alpha^{k} e^{-t\left(\alpha-\alpha^{-1}\right)} \frac{1}{2 \pi i} \int_{\Sigma_{c}} \frac{(-s)^{k} e^{t\left(s-s^{-1}\right)}}{s+\alpha^{-1}} d s=\frac{1}{2 \pi i} \int_{-A k^{\delta}}^{A k^{\delta}} \frac{e^{-\frac{1}{2}\left(y^{2}+x^{2}\right)}}{y+i x} d y\left(1+O\left(k^{-\frac{1}{2}+2 \delta}\right)\right)+O\left(e^{-c k^{2 \delta}}\right) .
$$

Thus from (10.154) and (10.155), we obtain

$$
\begin{array}{lll}
\lim _{k \rightarrow \infty} e^{-\alpha t}(-\alpha)^{k} \pi_{k}\left(-\alpha^{-1} ; t\right) & =\frac{1}{2 \pi i} \int_{-\infty}^{\infty} \frac{e^{-\frac{1}{2}\left(y^{2}+x^{2}\right)}}{y+i x} d y, & x<0, \\
\lim _{k \rightarrow \infty} e^{-\alpha t}(-\alpha)^{k} \pi_{k}\left(-\alpha^{-1} ; t\right) & =\frac{1}{2 \pi i} \int_{-\infty}^{\infty} \frac{e^{-\frac{1}{2}\left(y^{2}+x^{2}\right)}}{y+i x} d y+1, & x>0 .
\end{array}
$$

The function $h(x):=\frac{1}{2 \pi i} \int_{-\infty}^{\infty} \frac{e^{-\frac{1}{2}\left(y^{2}+x^{2}\right)}}{y+i x} d y$ is smooth in $x>0$ and $x<0$. The derivative is $h^{\prime}(x)=\frac{1}{\sqrt{2 \pi}} e^{-\frac{1}{2} x^{2}}$. As $x \rightarrow \pm \infty, h(x) \rightarrow 0$. Therefore we see that

$$
\frac{1}{2 \pi i} \int_{-\infty}^{\infty} \frac{e^{-\frac{1}{2}\left(y^{2}+x^{2}\right)}}{y+i x} d y= \begin{cases}\frac{1}{\sqrt{2 \pi}} \int_{-\infty}^{x} e^{-\frac{1}{2} y^{2}} d y, & x<0 \\ \frac{1}{\sqrt{2 \pi}} \int_{\infty}^{x} e^{-\frac{1}{2} y^{2}} d y, & x>0 .\end{cases}
$$

Thus we proved Proposition 5.8. 


\section{References}

[1] D. Aldous and P. Diaconis. Longest increasing subsequences: from patience sorting to the Baik-DeiftJohansson theorem. Bull. Amer. Math. Soc. (N.S.), 36(4):413-432, 1999.

[2] J. Baik. Riemann-Hilbert Problems and Random Permutations. PhD thesis, Courant Institute of Mathematical Sciences, 1999.

[3] J. Baik, P. Deift, and K. Johansson. On the distribution of the length of the longest increasing subsequence of random permutations. J. Amer. Math. Soc., 12(4):1119-1178, 1999.

[4] J. Baik, P. Deift, and K. Johansson. On the distribution of the length of the second row of a Young disgram under Plancherel measure. Geom. Funct. Anal., 10(4):702-731, 2000.

[5] J. Baik, P. Deift, and E. Rains. A Fredholm determinant identy and the convergence of moments for random Young tableaux. http://xxx.lanl.gov/abs/math.CO/0012117.

[6] J. Baik and E. M. Rains. Limiting distributions in stationary growth models. J. Statist. Phys., 100(3/4):523-541, 2000.

[7] J. Baik and E. M. Rains. Algebraic aspects of increasing subsequences. http://xxx.lanl.gov/abs/ math.CO/9905083, to appear in Duke Math. J.

[8] J. Baik and E. M. Rains. Symmetrized random permutations. http://xxx.lanl.gov/abs/math.CO/9910019, to appear in MSRI volume on Random Matrix Models and Their Applications.

[9] A. Borodin. Longest increasing subsequences of random colored permutations. Electron. J. Combin., 6(1):R13, 1999.

[10] A. Borodin, A. Okounkov, and G. Olshanski. On asymptotics of Plancherel measures for symmetric groups. J. Amer. Math. Soc., 13(3):481-515, 2000.

[11] P. Deift. Orthogonal polynomials and random matrices: a Riemann-Hilbert approach, volume 3 of Courant lecture notes in mathematics. CIMS, New York, NY, 1999.

[12] P. Deift. Integrable systems and combinatorial theory. Notices Amer. Math. Soc., 47(6):631-640, 2000.

[13] P. Deift, T. Kriecherbauer, K. McLaughlin, S. Venakides, and X. Zhou. Asymptotics for polynomials orthogonal with respect to varying exponential weights. Internat. Math. Res. Notices, 16:759-782, 1997.

[14] P. Deift, T. Kriecherbauer, K. McLaughlin, S. Venakides, and X. Zhou. Strong asymptotics of orthogonal polynomials with respect to exponential weights. Comm. Pure Appl. Math., 52(12):1491-1552, 1999.

[15] P. Deift, T. Kriecherbauer, K. McLaughlin, S. Venakides, and X. Zhou. Uniform asymptotics for polynomials orthogonal with respect to varying exponential weights and applications to universality questions in random matrix theory. Comm. Pure Appl. Math., 52(11):1335-1425, 1999. 
[16] P. Deift, S. Venakides, and X. Zhou. The collisionless shock region for the long-time behavior of solutions of the KdV equation. Comm. Pure Appl. Math., 47(2):199-206, 1994.

[17] P. Deift, S. Venakides, and X. Zhou. New results in small dispersion KdV by an extension of the steepest descent method for Riemann-Hilbert problems. Internat. Math. Res. Notices, 6:285-299, 1997.

[18] P. Deift and X. Zhou. A steepest descent method for oscillatory Riemman-Hilbert problems. Asymptotics for the MKdV equation. Ann. of Math., 137:295-368, 1993.

[19] P. Deift and X. Zhou. Asymptotics for the Painlevé II equation. Comm. Pure Appl. Math., 48:277-337, 1995.

[20] H. Flaschka and A. Newell. Monodromy and spectrum preserving deformations, I. Comm. Math. Phys., 76(1):67-116, 1980.

[21] A. Fokas, A. Its, and V. Kitaev. Discrete Painlevé equations and their appearance in quantum gravity. Comm. Math. Phys., 142:313-344, 1991.

[22] A. Fokas and X. Zhou. On the solvability of Painlevé II and IV. Comm. Math. Phys., 144:601-622, 1992.

[23] P. Forrester. Random walks and random permutations. http://xxx.lanl.gov/abs/math.CO/9907037.

[24] P. Forrester and E. Rains. Inter-relationships between orthogonal, unitary and symplectic matrix ensembles. http://xxx.lanl.gov/abs/solv-int/9907008.

[25] J. Gravner, C. Tracy, and H. Widom. Limit theorems for height fluctuations in a class of discrete space and time growth models. http://xxx.lanl.gov/abs/math.PR/0005133.

[26] C. Greene. An extension of Schensted's theorem. Adv. in Math., 14:254-265, 1974.

[27] S. Hastings and J. McLeod. A boundary value problem associated with the second Painlevé transcendent and the Korteweg de Vries equation. Arch. Rational Mech. Anal., 73:31-51, 1980.

[28] A. Its, C. Tracy, and H. Widom. Random words, Toeplitz determinants and integrable systems. I. http://xxx.lanl.gov/abs/math.CO/9909169.

[29] M. Jimbo, T. Miwa, and K. Ueno. Monodromy preserving deformations of linear ordinary differential equations with rational coefficients, I. General theory and $\tau$-function. Phys. D, 2(2):306-352, 1981.

[30] K. Johansson. The longest increasing subsequence in a random permutation and a unitary random matrix model. Math. Res. Lett., 5(1-2):63-82, 1998.

[31] K. Johansson. Shape fluctuations and random matrices. Comm. Math. Phys., 209(2):437-476, 2000.

[32] K. Johansson. Discrete orthogonal polynomial ensembles and the Plancherel measure. http://xxx.lanl.gov/abs/math.CO/9906120, to appear in Ann. Math. 
[33] D. E. Knuth. Sorting and Searching, volume 3 of The Art of Computer Programming. Addison Wesley, Reading, Mass., second edition, 1973.

[34] G. Kuperberg. Random words, quantum statistics, central limits, random matrices. http://xxx.lanl.gov/abs/math.PR/9909104.

[35] B. Logan and L. Shepp. A variational problem for random Young tableaux. Adv. in Math., 26:206-222, 1977.

[36] M. Mehta. Random matrices. Academic Press, San Diego, 2nd edition, 1991.

[37] A. M. Odlyzko and E. M. Rains. On longest increasing subsequences in random permutations. In Analysis, geometry, number theory: the mathematics of Leon Ehrenpreis (Philadelphia, PA, 1998), pages 439-451. Amer. Math. Soc., 2000.

[38] A. Okounkov. Random matrices and random permutations. Internat. Math. Res. Notices, 20:1043, 2000.

[39] A. Regev. Asymptotic values for degrees associated with strips of Young diagrams. Adv. in Math., 41:115$136,1981$.

[40] C. Schensted. Longest increasing and decreasing subsequences. Canad. J. Math., 13:179-191, 1961.

[41] H. Spohn and M. Prähofer. Statistical self-similarity of one-dimensional growth processes. Phys. Rev. Lett., 84:4882-4885, 2000.

[42] H. Spohn and M. Prähofer. Universal distributions for growth processes in $1+1$ dimensions and random matrices. Physica A, 279:342-352, 2000.

[43] R. Stanley. Generalized riffle shuffles and quasisymmetric functions. http://xxx.lanl.gov/abs/ math.CO/9912025.

[44] C. Tracy and H. Widom. On the distribution of the lengths of the longest monotone subsequences in random words. http://xxx.lanl.gov/abs/math.CO/9904042.

[45] C. Tracy and H. Widom. Level-spacing distributions and the Airy kernel. Comm. Math. Phys., 159:151-174, 1994.

[46] C. Tracy and H. Widom. On orthogonal and symplectic matrix ensembles. Comm. Math. Phys., 177:727$754,1996$.

[47] C. Tracy and H. Widom. Random unitary matrices, permutations and Painlevé. Comm. Math. Phys., 207(3):665-685, 1999.

[48] A. Vershik and S. Kerov. Asymptotics of the Plancherel measure of the symmetric group and the limiting form of Young tables. Soviet Math. Dokl., 18:527-531, 1977. 